Aus der Abteilung Operative Intensivmedizin

(Prof. Dr. med. M. Quintel)

des Zentrum Anaesthesiologie, Rettungs- und Intensivmedizin

in der Medizinischen Fakultät der Universität Göttingen

\author{
Experimentelle und klinische \\ Untersuchung der \\ elektrischen Impedanztomographie \\ zur regionalen Lungenfunktionsprüfung \\ beatmeter Patienten
}

\author{
Habilitationsschrift \\ (kumulatives Verfahren) \\ zur Erlangung der Venia legendi \\ in der Medizinischen Fakultät \\ der Georg-August Universität zu Göttingen
}

von

Dr. med. José Hinz, DEAA

Göttingen 2005 


\section{Inhaltsverzeichnis}

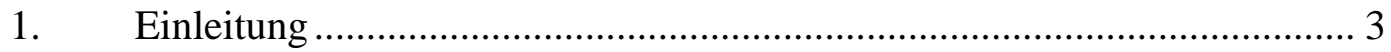

2. Material und Methoden ........................................................................ 9

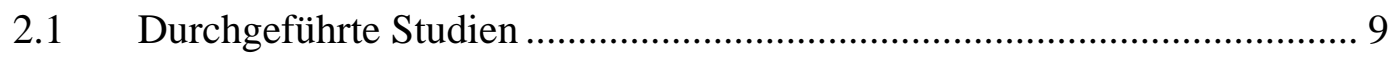

2.2 Elektronenstrahl-Computertomogram (EBCT)..................................... 16

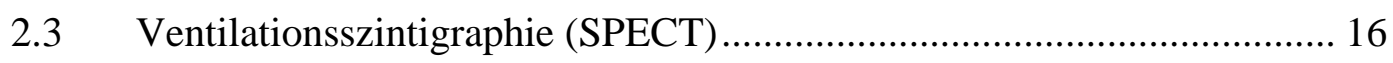

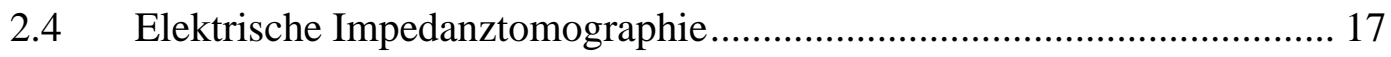

2.4.1 Funktionelle Impedanztomographie.....................................................18

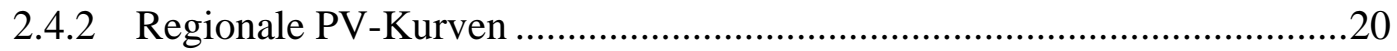

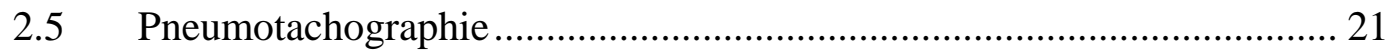

2.6 Offener Stickstoffauswaschvorgang ....................................................... 22

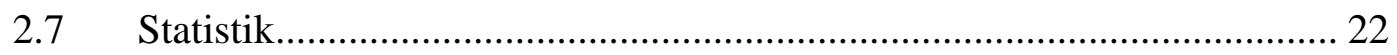

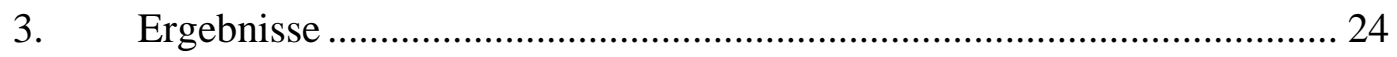

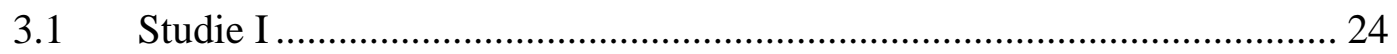

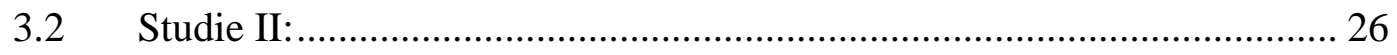

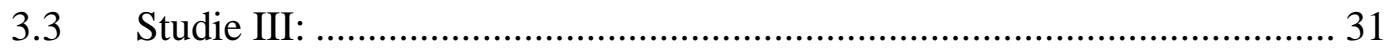

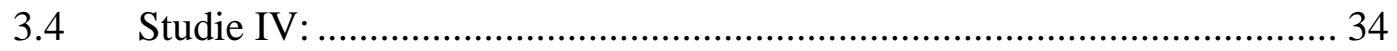

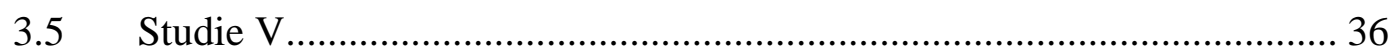

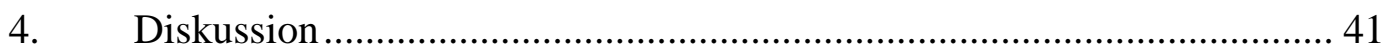

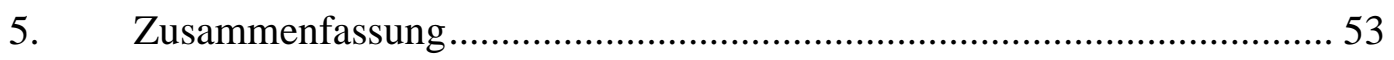




\section{Einleitung}

Die maschinelle Beatmung ist Bestandteil moderner medizinischer Therapiekonzepte, um bei Störungen des pulmonalen Gasaustausches eine ausreichende Versorgung des Patienten mit Sauerstoff und die Elimination von Kohlendioxid zu gewährleisten. Bei einer Erschöpfung der Atemmuskulatur als Folge akuter oder chronischen Erkrankung mit erhöhter Atemarbeit wird durch eine maschinelle Beatmung die Atemmuskulatur entlastet (Wrigge et al. 1999). Dies erlaubt eine Erholung der Atemmuskulatur, die neben der Therapie der Grunderkrankung für eine Rekonvaleszenz des Patienten unumgänglich ist. Bei Patienten mit schweren lebensbedrohlichen Erkrankungen wie Sepsis, Polytrauma oder isolierten Lungenversagen gehört die maschinelle Beatmung zum etablierten Behandlungskonzept (Andrews et al. 2005, Dellinger et al. 2004, Hudson und Steinberg 1999).

Die maschinelle Beatmung führt jedoch auch regelmäßig zu einer Verschlechterung der Lungenfunktion. Sie ist gekennzeichnet durch eine Zunahme der alveolo-kapillären Sauerstoffdifferenz, so dass eine inspiratorische Sauerstofffraktion $\left(\mathrm{F}_{\mathrm{i}} \mathrm{O}_{2}\right)$ von mindestens 0,3 für die Beatmung empfohlen wird (Sykes et al. 1965). Es wurde dabei ein kausaler Zusammenhang zwischen der maschinellen Beatmung, schlechter werdender Lungenfunktion und dem Auftreten von nicht beatmeten Lungenregionen (Atelektasen) diskutiert. Computertomographische Untersuchungen des Thorax unterstrichen diesen Zusammenhang (Brismar et al. 1985, Gunnarsson et al. 1991, Hedenstierna et al. 1986). Weiterhin wurde gezeigt, dass die Größe der Atelektasen mit dem intrapulmonalen Rechts-Links-Shunts korreliert (Bendixen et al. 1963). Eine 
besondere Bedeutung für die Entstehung von Atelektasen kommt der inspiratorischen Sauerstofffraktion $\left(\mathrm{F}_{\mathrm{i}} \mathrm{O}_{2}\right)$ zu. Wird bei einer Narkoseeinleitung auf eine Präoxygenierung mit reinem Sauerstoff $\left(\mathrm{F}_{\mathrm{i}} \mathrm{O}_{2}=1,0\right)$ verzichtet, oder reduziert man die $\mathrm{F}_{\mathrm{i}} \mathrm{O}_{2}$ direkt nach der endotrachealen Intubation auf 0 ,3, so ist die Größe der Atelektasen signifikant kleiner als nach Präoxygenierung und dreiminütiger Beatmung mit reinem Sauerstoff (Reber et al. 1996). Eröffnet man atelektatische Lungenareale während maschineller Beatmung durch die kurzfristige Anwendung eines erhöhten Atemwegsdruck $\left(\mathrm{P}_{\mathrm{AW}}\right)$, so ist die Geschwindigkeit des erneuten Auftretens sowie die Größe der entstehenden Atelektasen ebenfalls von der $\mathrm{F}_{\mathrm{i}} \mathrm{O}_{2}$ abhängig (Rothen et al. 1995). Atelektasen erklären jedoch nicht nur die Zunahme der alveolo-kapillären Sauerstoffdifferenz von Patienten in Narkose, sondern sie kennzeichnen auch das akute Lungenversagen bei Patienten in seiner schwersten Ausprägung, dem „Acute Respiratory Distress Syndrome“ (ARDS) (Bernard et al. 1994). Großflächige dorso-basale Atelektasen sind beim ARDS typischerweise nachweisbar (Gattinoni et al. 1986, Gattinoni et al. 1988). Wie bei lungengesunden Patienten in Narkose korreliert auch beim ARDS der intra-pulmonale Rechts-Links-Shunt mit der Größe der Atelektasen (Gattinoni et al. 1988). Das Ziel jeder Beatmung muss daher sein, die Entstehung von Atelektasen zu verhindern und bereits vorhandene Atelektasen wieder zu eröffnen und für den Gasaustausch zu rekrutieren. Lachmann formulierte in einem Editorial den Grundsatz „Open up the lung and keep the lung open“ (Lachmann 1992). Deshalb wurden verschiedenste Vorschläge gemacht, um Atelektasen zu vermeiden oder zu rekrutieren. Dazu gehören das „open lung“ Konzept (Lachmann 1992), die superpositionierte 
Spontanatmung während maschineller Beatmung (Putensen et al. 2001), die maschinelle Beatmung mit inversen Inspiration-Exspiration-Zeitverhältnissen (Sydow et al. 1994), die Bauchlagerung des Patienten (Flatten et al. 1998), die nicht invasive Beatmung (Brochard 2000) oder die maschinelle Beatmung mit positiv end-exspiratorischen Drucken (PEEP) (Neumann et al. 1998b).

Die Beatmung schädigt jedoch bei inadäquater PEEP und/oder Plateaudruck die Lunge sekundär durch zyklisches Kollabieren und Wiedereröffnen von Alveolen (Dreyfuss und Saumon 1998, Pinhu et al. 2003). Die dabei entstehenden Scherkräfte führen zur Freisetzung von inflammatorischen Zytokinen (Chiumello et al. 1999, Ranieri et al. 1999) und verursachen dadurch sekundäre Schäden an anderen Organen (Andrews et al. 2005, Plotz et al. 2003, Plotz et al. 2004, Slutsky und Tremblay 1998). Inadäquate hohe Beatmungsdrucke und hohe Tidalvolumina $\left(\mathrm{V}_{\mathrm{T}}\right)$, die zur Eröffnung kollabierter Lungenregionen bei Rekrutierungsmanövern eingesetzt werden, können durch eine Überdehnung des Lungenparenchyms und konsekutivem Einriss von Lungenepithelien ebenfalls sekundäre Lungenschäden hervorrufen und damit $\mathrm{zu}$ einer progredienten Verschlechterung der Lungenfunktion führen (Downey und Granton 1997, Kacmarek 1999, Matamis et al. 1984, Pelosi und Gattinoni 1996, Sykes 1991). Tatsächlich konnte gezeigt werden, dass eine Beatmung mit hohem positivem end-exspiratorischem Atemwegsdruck (PEEP) und kleinen Tidalvolumina $\left(\mathrm{V}_{\mathrm{T}}\right)$, welche die oben beschriebenen Scherkräfte minimiert und den Plateaudruck reduziert, die Prognose von Patienten mit akutem Lungenversagen günstig beeinflusst (Amato et al. 1998, The Acute Respiratory Distress Syndrome Network 2000). Nach bisherigem Kenntnisstand kann der adäquate PEEP und 
Plateaudruck aus Druck-Volumen-Kurven (PV-Kurve) der Lunge bestimmt werden, so dass die Messung der Atemmechanik von Bedeutung ist (Gattinoni et al. 1984). Der PEEP wird aus dem unteren Inflektionspunkt (LIP) der PV-Kurve bestimmt, wohingegen der Plateaudruck den oberen Inflektionspunkt (UIP) nicht überschreiten sollte. Kritisch bleibt jedoch anzumerken, dass Druck-VolumenKurven der gesamten Lunge nicht repräsentativ sind für alle Lungenregionen, da sie sich bezüglich der Atemmechaniken regional unterscheiden. In einer Studie konnte durch Computertomographie (CT) gezeigt werden, dass Patienten mit ARDS regional unterschiedliche Compliances aufweisen (Puybasset et al. 2000). Deshalb wurde bei beatmeten Patienten mit akutem Lungenversagen (ALI) und ARDS empfohlen, zur Respiratoreinstellung regionale pulmonale Inhomogenitäten in die Überlegungen der atemmechanischen Messungen mit einzubeziehen und dass Techniken wünschenswert sind, die die regionale Funktion der Lunge überwachen (Rouby et al. 2002).

Die Computertomographie des Thorax ist die sensitivste Methode zur Erkennung von Atelektasen und Überblähungen der Lunge sowie der Überwachung der regionalen Atemmechanik und derer Inhomogenität. Sie liefert anhand der Hounsfield-Einheiten (HU) Informationen über regionale Atelektasen und Lungenvolumen (Gattinoni et al. 1987). Der Nachteil dieses Verfahren ist die Strahlenbelastung des Patienten, die wiederholte oder sogar kontinuierliche CT-Untersuchungen zur Objektivierung von Respiratoreinstellungen einschränken. Bei einer weiteren Methode zur Messung der regionalen Lungenfunktion, der Ventilationsszintigraphie wird ein Radiopharmakon (Tc-99m Albumin-Mikropartikel) inhaliert und mit hochauflösenden Kollimatoren aus 
verschiedenen Kameraprojektionen die Aktivität des Radiopharmakons bestimmt und daraus die regionale Ventilation berechnet. Ein Nachteil dieses Verfahren ist die lange Messdauer, so dass rasche Veränderungen der regionalen Lungenfunktion, wie Bildung von Atelektasen nicht sicher im zeitlichen Verlauf beurteilt werden können (Neumann et al. 1998a, Neumann et al. 1998b). Weitere experimentelle jedoch klinisch nicht eingeführte Techniken sind die multiple Inertgas Eliminationstechnik (MIGET) zur Bestimmung von VentilationsPerfusions-Verhältnissen (Wagner et al. 1974) und die Magnetresonanztomographie (van Beek et al. 2004).

Aus dem bisher Gesagtem wird deutlich, dass zur Überwachung, Rekrutierung und Vermeidung von Atelektasen und Lungenüberdehnung eine kontinuierliche, bettseitige Überwachung der regionalen Lungenfunktion wünschenswert ist (Dreyfuss und Saumon 1998). Es stehen mehrere Verfahren zur Verfügung, mit denen die regionale Lungenfunktion untersucht werden kann. Allen diesen Methoden ist gemeinsam, dass die Untersuchung nicht bettseitig erfolgen kann, so dass ein Transport des Intensivpatienten erforderlich wird. Kritisch kranke Patienten haben jedoch während eines Transportes innerhalb des Krankenhauses eine erhöhte Morbidität und Mortalität (Andrews et al. 1990, Bercault et al. 2005, Smith et al. 1990, Warren et al. 2004). Ein Ausweg aus diesem Dilemma könnte die in den frühen 80er Jahren entwickelte elektrische Impedanztomographie (EIT) sein (Barber und Brown 1984). Das Prinzip der EIT basiert auf der Einspeisung von Wechselströmen mit geringer Amplitude (5mA p-p) und Spannungsmessung über Oberflächenelektroden, die am Thorax des Patienten angebracht sind. Hierdurch bestimmt die EIT regionale 
Impedanzänderungen innerhalb des Thorax. Da die Änderung der Impedanz von der Änderung des Luftgehaltes abhängig ist, ermöglicht die EIT eventuell eine bettseitige, kontinuierliche und strahlungsfreie Messung der regionalen Ventilation. Die EIT wurde bisher experimentell zum nicht invasiven Lungenmonitoring verwendet (Frerichs 2000). Es konnte gezeigt werden, dass sie bekannte physiologische Effekte reproduzieren kann (Adler et al. 1997, Adler et al. 1998) Die Überprüfung der EIT mit einem klinisch etablierten Verfahren der Luftgehalts- und Ventilationsmessung fehlt jedoch bis heute. Ein positiver Vergleich der nicht invasiven EIT mit Referenzverfahren wäre bei beatmeten Patienten hilfreich, um die regionalen Lungenfunktion bettseitig zu überwachen, die Einstellung von Beatmungsgeräten zu optimieren und Atelektasen und Lungenüberblähung zu verhindern. Kombiniert mit einer Atemwegsdruckmessung $\left(\mathrm{P}_{\mathrm{AW}}\right)$ könnte die EIT bettseitig regionale PV-Kurven messen. Das Ziel dieser Habilitationsarbeit war deshalb die Überprüfung der EIT mit klinisch etablierten Verfahren der globalen und regionalen Lungenvolumenund Ventilationsmessung (CT, Ventilationsszintigraphie und offener Stickstoffauswaschvorgang). Desweiteren sollte ein Verfahren entwickelt werden, dass die bettseitige Überwachung des Einflusses von PEEP auf die regionale Ventilation ermöglicht. Zusätzlich sollte bettseitige die kontinuierliche Messung der regionalen Atemmechanik durch regionale PV-Kurven ermöglicht werden. 


\section{Material und Methoden}

\subsection{Durchgeführte Studien}

Die Habilitation wurde in enger Zusammenarbeit mit der Abteilung Anästhesiologische Forschung (Prof. Dr. med. G. Hellige) des Zentrum Anaesthesiologie, Rettungs- und Intensivmedizin durchgeführt und basiert auf den folgenden Originalarbeiten, die im Text mit den Ziffern I - V bezeichnet werden:

I. Inez Frerichs, José Hinz, Herrmann P, Weisser G, Hahn G, Taras Dudykevych, Michael Quintel, Gerhard Hellige (2002): Detection of local lung air content by electrical impedance tomography compared with electron beam CT. J Appl Physiol 93(2), 660-666

II. José Hinz, Peter Neumann, Taras Dudykevych, Lars Goran Andersson, Herrman Wrigge, Hilmar Burchardi, Goran Hedenstierna (2003): Regional ventilation by Electrical Impedance Tomography-A comparison with ventilation scintigraphy in pigs. Chest 124, 314-322

III. José Hinz, Günter Hahn, Peter Neumann P, Michael Sydow, Peter Mohrenweiser, Gerhard Hellige, Hilmar Burchardi (2003): End-expiratory lung impedance change enables bedside monitoring of end-expiratory lung volume change. Intensive Care Med 1, 37-43

IV. José Hinz, Peter Mohrenweiser, Peter Neumann, Günter Hahn, Michael Sydow, Gerhard Hellige, Hilmar Burchardi (2005): Effects of positive end expiratory pressure on regional ventilation in mechanically ventilated patients. Eur J Anaesth 22(11), 817825

V. José Hinz, Onnen Moerer, Peter Neumann, Taras Dudykevych, Inez Frerichs, Gerhard Hellige, Michael Quintel (voraussichtlich 2006): Regional pulmonary pressure volume curves in mechanically ventilated patients with acute respiratory failure measured by Electrical Impedance Tomography. Acta Anaesthesiol Scand (akzeptiert 2005) 
Alle Studien wurden durch die zuständigen Ethikkommissionen genehmigt. Einwilligungsfähige Patienten stimmten nach ausführlicher Aufklärung schriftlich der Teilnahme an der Studie zu. Bei nicht einwilligungsfähigen Patienten erklärten die nächsten Angehörigen oder ein vom Gericht bestimmter Betreuer den mutmasslichen Willen des Patienten zur Teilnahme in die Studie.

Untersucht wurden maschinell beatmete Schweine mit gesunden und Ölsäure geschädigten Lungen als Modell einer ARDS Lunge. Desweiteren wurden maschinell beatmete Patienten mit akutem Lungenversagen in die Studien eingeschlossen. Die regionale Ventilation wurde zum einem bettseitig, nicht invasiv, strahlungsfrei mit der elektrischen Impedanztomographie und zum anderen invasiv, strahlenbelastend mit einem Elektronenstrahl-CT und der Ventilationsszintigraphie gemessen. Das globale end-exspiratorische Lungenvolumen wurde mit einem offenen Stickstoffauswaschvorgang gemessen. Die regionale Ventilation wurde nach dem Verfahren der funktionellen Impedanztomographie bestimmt (Hahn et al. 1996). Desweiteren wurde die regionale Atemmechanik durch eine neuartige Methode der Bestimmung von regionalen PV-Kurven aus zeitgleichen Messungen der elektrischen Impedanztomographie und des Atemwegdruckes bestimmt.

Die Studie I wurde durchgeführt im Labor des ElektronenstrahlComputertomogram (EBCT) der Universität Mannheim. Dort untersuchten wir an sechs maschinell beatmeten Schweinen mit gesunden Lungen die regionale Ventilation mit EIT (Goe-MF, EIT-Group Göttingen, Göttingen) und EBCT. Das 
EBCT diente wegen seiner hohen zeitlichen Auflösung als klinisches Referenzverfahren zur Messung der regionalen Ventilation. Änderungen im regionalen Luftgehalt und der regionalen Ventilation wurden durch Variation von drei PEEP und fünf Tidalvolumina generiert. Für jede Lungenhälfte wurden in der Elektrodenebene medioclavicular eine ventrale, mittlere und dorsale Regionen ausgewählt (s. Abbildung 1 Seite 11). Wir untersuchten zeitgleich die regionale Ventilation in diesen sechs Regionen mit der EIT und EBCT und verglichen die Ergebnisse.
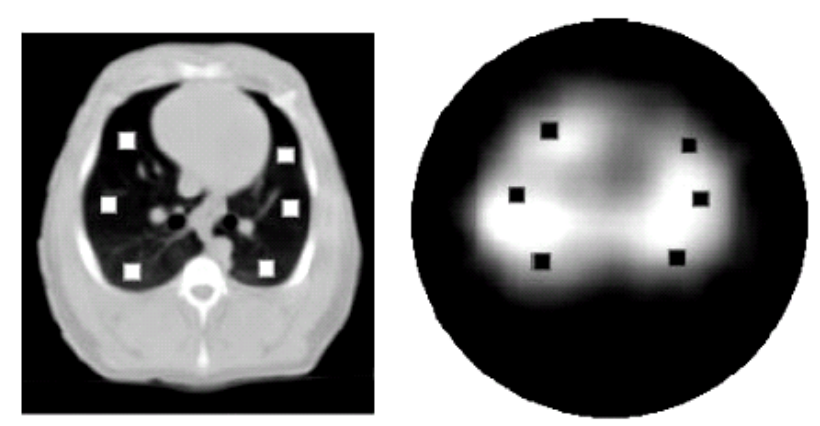

Abbildung 1: Auswahl der sechs Messregionen am Elektronenstrahl-CT (links) und der funktionelle Impedanztomographie (rechts) am Beispiel eines maschinell beatmeten Schweins (Frerichs et al. 2002a).

Studie II wurde durchgeführt im Tierversuchslabor der Abteilung klinische Physiologie der Universität Uppsala, Schweden. Es wurden zwölf Schweine anästhesiert und maschinell beatmet. Ein akutes Lungenversagen wurde durch zentralvenöse Applikation von Ölsäure (Schuster 1994) induziert. Das Ölsäuremodell eignet sich, um besonders schwer geschädigte Lungen zu erzeugen 
und zu untersuchen (Neumann und Hedenstierna 2001). Die Dosierung der Ölsäure wurde durch intermittierende Blutgasanalysen gesteuert, um eine akute Lungenschädigung mit einem $\mathrm{PaO}_{2} / \mathrm{F}_{\mathrm{i}} \mathrm{O}_{2}$ von $200 \mathrm{~mm} \mathrm{Hg}$ zu erreichen. Nach Stabilisierung der Lungenschädigung untersuchten wir zeitgleich die regionale Ventilation in 20 koronalen Segmenten einer vier Zentimeter dicken transversalen Schicht mit der EIT (Goe-MF, EIT-Group Göttingen, Göttingen) und Ventilationsszintigraphie (s. Abbildung 2 Seite 12). Die Ventilationsszintigraphie diente als klinisch etabliertes Referenzverfahren zur Messung der regionalen Ventilation bei diesem ARDS Modell.

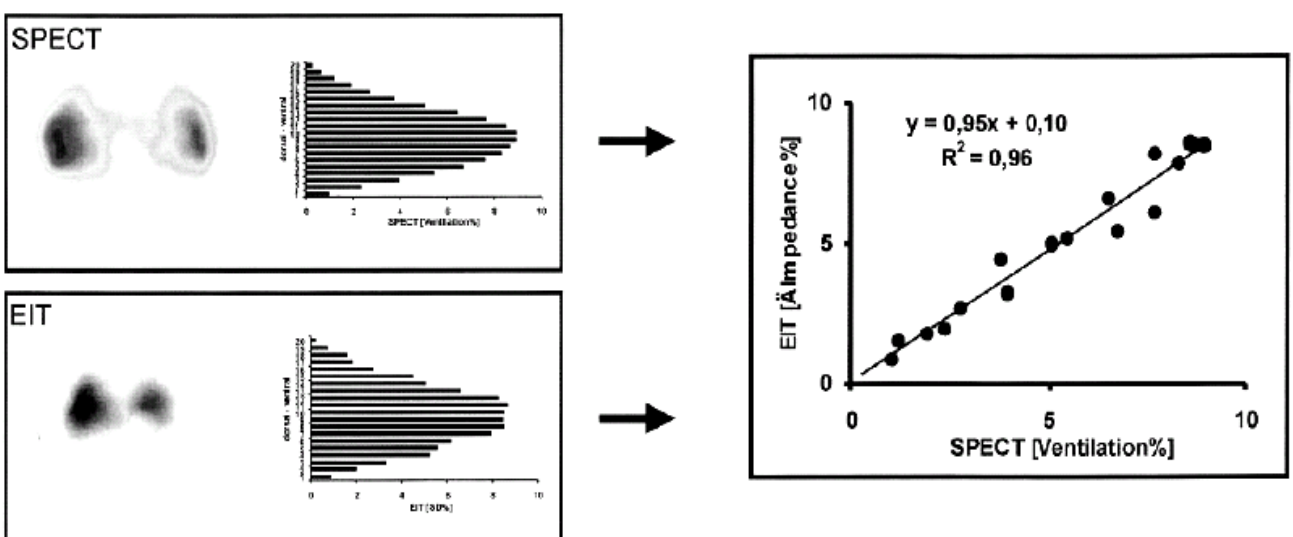

Abbildung 2: Beispiel des Auswerteverfahren Nach Stabilisierung der Lungenschädigung verglichen wir zeitgleich die regionale Ventilation in 20 koronalen Segmenten einer vier Zentimeter transversalen Schicht mit der elektrischen Impedanztomographie (EIT) und Ventilationsszintigraphie (SPECT) (Hinz et al. 2003b).

Studie III führten wir auf den Intensivstationen des Zentrums Anästhesiologie, Rettungs- und Intensivmedizin im Klinikum Göttingen durch. Bei zehn maschinell beatmeten Patienten mit einem akutem Lungenversagen wurde vier verschiedene PEEP gewählt und das sich verändernde globale end- 
exspiratorische Lungenvolumen mit der EIT (APT System MK1, IBEES, Sheffield, UK) (s. Abbildung 3 Seite 13) und einem offenen Stickstoffauswaschvorgang als Referenzverfahren untersucht und verglichen.

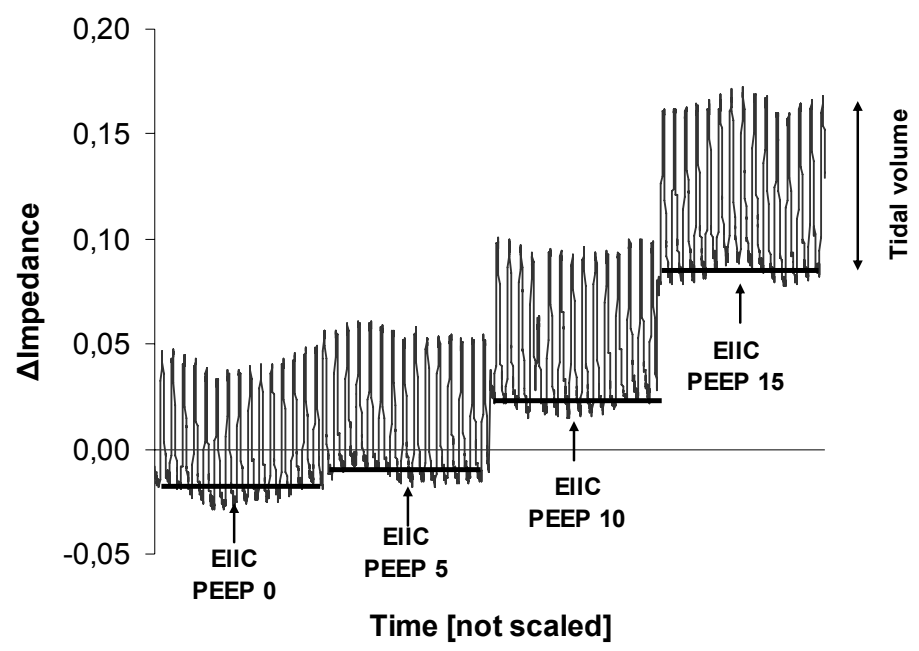

\begin{abstract}
Abbildung 3: Bestimmung der Änderung des endexspiratorischen Lungenvolumens bei vier verschiedenen PEEPs aus globalen end-exspiratorischen Impedanzzeitverläufen (EILC) der elektrischen Impedanztomographie (Hinz et al. 2003a).
\end{abstract}

Studie IV führten wir ebenfalls auf der Intensivstation des Zentrums Anästhesiologie, Rettungs- und Intensivmedizin im Klinikum Göttingen durch. Wir untersuchten an acht wegen eines akuten Lungenversagens maschinell beatmeten Patienten die Effekte von PEEP auf die regionale Ventilation mit der inzwischen überprüften elektrischen Impedanztomographie. Hierzu wurde alle 40 Minuten der PEEP von 0 mbar beginnend in Stufen von 5 mbar auf bis 15 mbar erhöht und anschliessend auf 0 mbar zurückgegangen. Wir untersuchten die regionale Ventilation mit der EIT (APT System MK1, IBEES, Sheffield, UK) in 
912 Regionen eines Thoraxquerschnitt in der Elektrodenebene. Die 912 regionalen Ventilationen wurden in vier Ventilationsgruppen (nicht ventiliert, schlecht ventiliert, moderat ventiliert und gut ventiliert) eingeteilt und bei verschiedenen PEEPs untersucht (s. Abbildung 4 Seite 14).

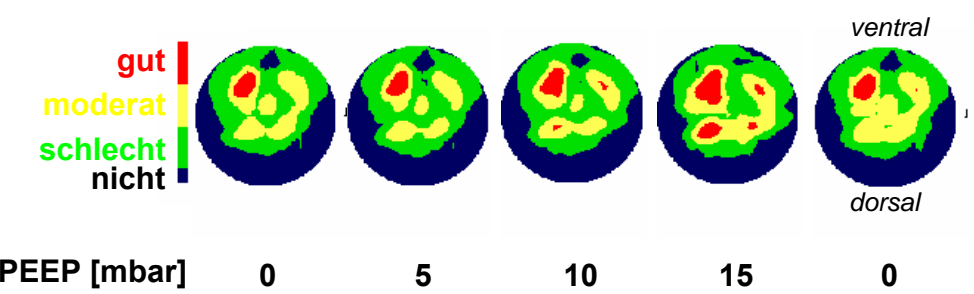

\begin{abstract}
Abbildung 4: Beispiele regionaler Ventilationsgruppen (nicht, schlecht, moderat, gut) bei fünf verschiedenen PEEPs in einer transversalen Thoraxschicht im 6. Intercostalraum eines beatmeten Patienten gemessen mit der funktionellen Impedanztomographie (f-EIT) (Hahn et al. 1995, Hahn et al. 1997). Die Darstellung der f-EIT basiert auf der Berechnung der Standardabweichung der Impedanzänderung in jeder der 912 Bildregion eines EIT-Bildes. Eine Erhöhung des PEEP resultierte in einer Zunahme der Ventilation in dorsalen Anteilen des Thoraxquerschnitt und basiert auf einer Abnahme atelektatischer Lungenregionen (Hinz et al. 2005).
\end{abstract}

Studie V führten wir ebenfalls auf der Intensivstation des Zentrum Anästhesiologie, Rettungs- und Intensivmedizin im Klinikum Göttingen durch. Wir untersuchten bettseitig die regionale Atemmechanik von neun beatmeten Patienten mit akutem Lungenversagen durch die neuartige Kombination von EIT und Atemwegsdruck zur Generierung von regionalen PV-Kurven. Während eines inspiratorischen Low-Flow-Manöver (Mankikian et al. 1983) untersuchten wir 
zeitgleich konventionelle und regionale PV-Kurven und vermuteten eine ausgeprägte Heterogenität der regionalen PV-Kurven.

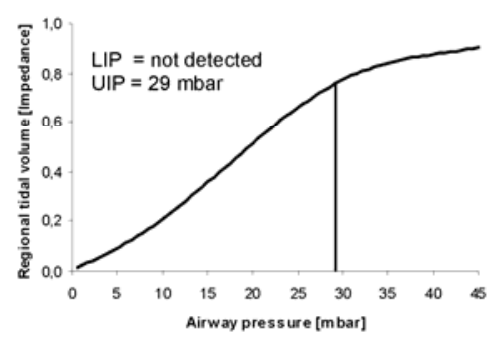

median left

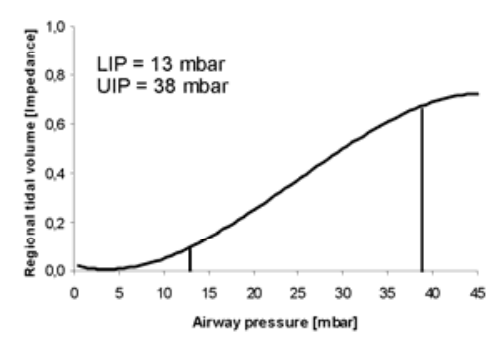

dorsal left

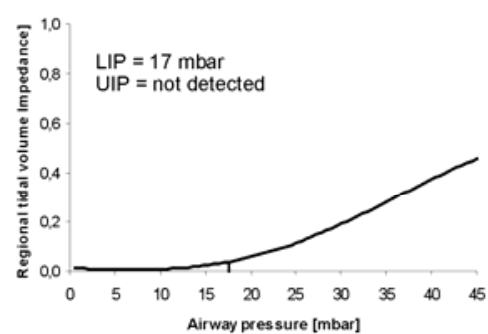

ventral right

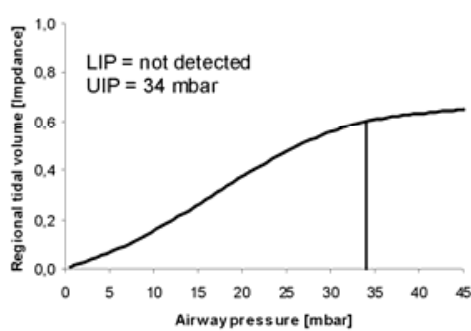

median right

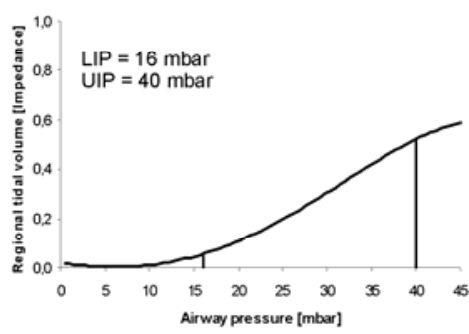

dorsal right

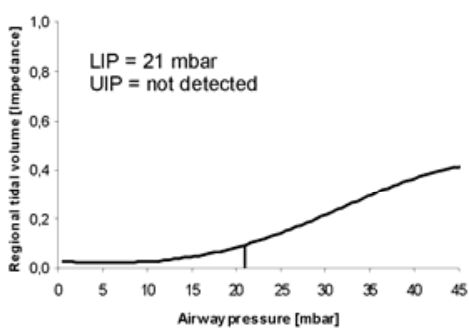

Abbildung 5: Sechs Beispiele aus insgesamt 912 möglichen regionalen PV-Kurven. PV-Kurven zeigen beim Lungenversagen üblicherweise einen charakteristischen sigmoiden Verlauf. Am Übergang vom initial flachen zum steilen Teil der Kurve findet man den unteren Inflektionspunkt (LIP), der den günstigsten PEEP definiert. Am Übergang vom steilen zum späten flachen Teil findet man den oberen Inflektionspunkt (UIP), der vom Plateaudruck nicht überschritten werden sollte. Gelegentlich konnten LIP und UIP jedoch nicht gefunden werden (Hinz et al. 2006). 


\subsection{Elektronenstrahl-Computertomogram}

Die Messung der regionalen Ventilation mit dem Referenzverfahren Elektronenstrahl-CT (EBCT) (EBCT C-150XP Imatron, San Francisco, CA) wurde an der Universität Mannheim im „multisclice“ Verfahren durchgeführt (Becker et al. 1998). Während einer Untersuchungsperiode von 12 Sekunden wurden 160 CT Schichten mit einer Untersuchungsgeschwindigkeit von 3,3 Schichten pro Sekunde untersucht. Die Auflösung des EBCT Bildes betrug 256*256 Bildpunkte. Regionen mit - 1000 bis -900 Hounsfield-Units (HU) wurden als überbläht und Regionen mit - 100 bis $+100 \mathrm{HU}$ als atelektatisch definiert. Wohingegen Regionen von -900 bis -500 HU als normal belüftet und von -400 bis -200 HU als minder belüftet definiert wurden (Gattinoni et al. 1987).

\subsection{Ventilationsszintigraphie}

Die Messung der regionalen Ventilation mit dem Referenzverfahren Ventilationsszintigraphie (SPECT) wurde mit ${ }^{99 \mathrm{~m}}$ Technetium markierten Karbonteilchen (Technegas ${ }^{\circledR}$, Tetley Medical Limited, NSW, Australia) in der Universität Uppsala, Schweden durchgeführt. Die Partikelgrösse beträgt $0,1 \mu \mathrm{m}$ und es wurde gezeigt, dass sich die Partikel pulmonal ähnlich der eines radioaktiven Gases verteilen (Burch et al. 1986). Dieses „Pseudogas“ wurde kontinuierlich mit einer an das Beatmungssystem verbundenen 2l Spritze über 
einen Zeitraum von 3-5 Minuten appliziert. Die SPECT Daten wurden in 64 Projektionen auf einer Doppelkopf Gamma-Kamera (Maxxus, General Electric Systems, Milwaukee, WI, USA) mit einer Bildaufnahmedauer von 15 Sekunden pro Projektion und einer Bildauflösung von 64*64 Bildpunkten gemessen. Das Atemminutenvolumen wurde mit Pneumotachographie bestimmt. Die regionale Ventilation wurde in einer vier Zentimeter Schicht in der EIT Elektrodenebene auf der Basis des gemessenen Atemminutenvolumen berechnet (Nuclear Diagnostics HERMES Workstation, Stockholm, Schweden). Die Schicht wurde in 20 gleichmässige koronare Schichten, die von der Wirbelsäule zum Sternum verliefen (s. Abbildung 2 S.12). Die regionale Ventilation wurde als Anteil der Gesamtventilation in der untersuchten Elektrodenebene berechnet und miteinander verglichen.

\subsection{Elektrische Impedanztomographie}

In allen Studien wurde die regionale Ventilation bettseitig nicht invasiv mit der elektrischen Impedanztomographie (EIT) (Barber und Brown 1984) in Kombination mit dem Auswertealgorithmus der funktionellen Impedanztomographie (Hahn et al. 1996) gemessen. Das Prinzip der EIT basiert auf der Einspeisung kleiner Wechselströme (5 mA p-p) und nachfolgender Spannungsmessung über 16 Oberflächenelektroden, die am Thorax angebracht sind (s. Abbildung 6 Seite 18). Aus den gemessenen Oberflächenspannungen lasssen sich in einem back-projection-Algorithmus relative Impedanzänderungen in 912 Regionen berechnen (Barber und Brown 1986). Die regionalen Impedanzänderungen sind zum überwiegenden Teil vom Luftgehalt zu einem 
geringen Teil vom pulsatilen Blutstrom abhängig (Faes et al. 1999). Bei den Untersuchungen wurden zwei unterschiedliche EIT-Geräte (APT System MK1, IBEES, Sheffield, UK und Goe-MF, EIT-Group Göttingen, Göttingen). Das APT System MK1 wurde in den 80er Jahren in Sheffield entwickelt und ist ein Gerät der ersten Generation. Das Goe-MF wurde in den letzten Jahren in der Abteilung Anästhesiologische Forschung (Prof. G. Hellige, Zentrum Anaesthesiologie, Rettungs- und Intensivmedizin) entwickelt. Es zeichnet sich durch ein deutlich verbessertes Signal-Rausch-Verhältnis aus (Hahn et al. 2000, Hahn et al. 2001).

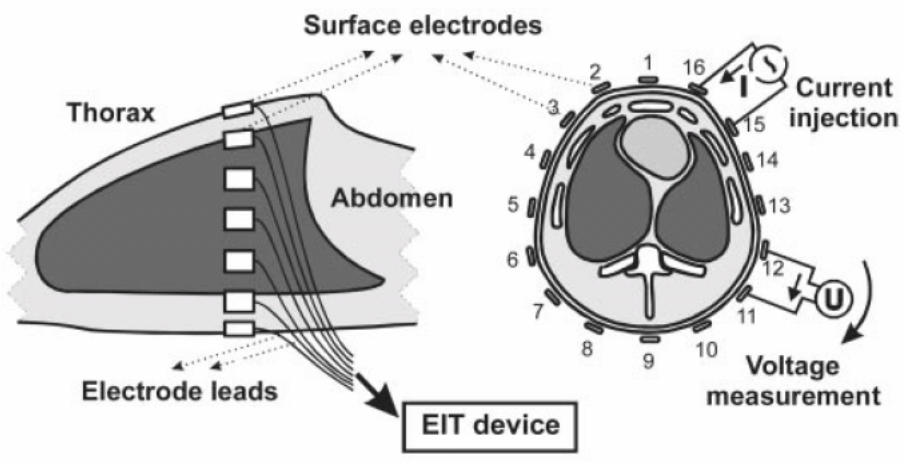

Abbildung 6: Prinzip der elektrischen Impedanztomographie Über ein Paar von 16 Oberflächenelektroden wird ein kleiner Strom eingespeist (current injection) und über den nicht beteiligten 13 Oberflächenelektroden die resultierende Oberflächenspannung gemessen (Voltage measurement). Umlaufend wird jedes benachbarte Oberflächenpaar als Einspeisungselektroden genutzt, so dass 208 Oberflächenspannungen (16 Einspeisungen* 13 Oberflächenspannungen) gemessen werden (Frerichs et al. 2002a).

\subsubsection{Funktionelle Impedanztomographie}

Die regionale Ventilation wurde mit der Methode der funktionellen Impedanztomographie (f-EIT) (Hahn et al. 1996) bestimmt. Das Prinzip basiert 
auf der Kompression von Messreihen regionaler Impedanzänderungen über einen Zeitraum (i.d.R. 1000 EIT-Bilder über 100 Sekunden) und der Berechnung der Variation der regionalen Impedanzänderung. Die Variation der Impedanzänderung wird aus der Standardabweichung der regionalen Impedanzänderung der EIT-Bildserie für jeden der 912 Bildpunkte berechnet. Regionen mit grosser regionaler Ventilation weisen eine grosse Standardabweichung und Regionen mit geringer regionaler Ventilation zeigen eine geringe Variation. Ein f-EIT-Bild stellt somit die regionale Impedanzvariation (Standardabweichung) als Parameter für regionale Ventilation dar (s. Abbildung 7, Seite 19).

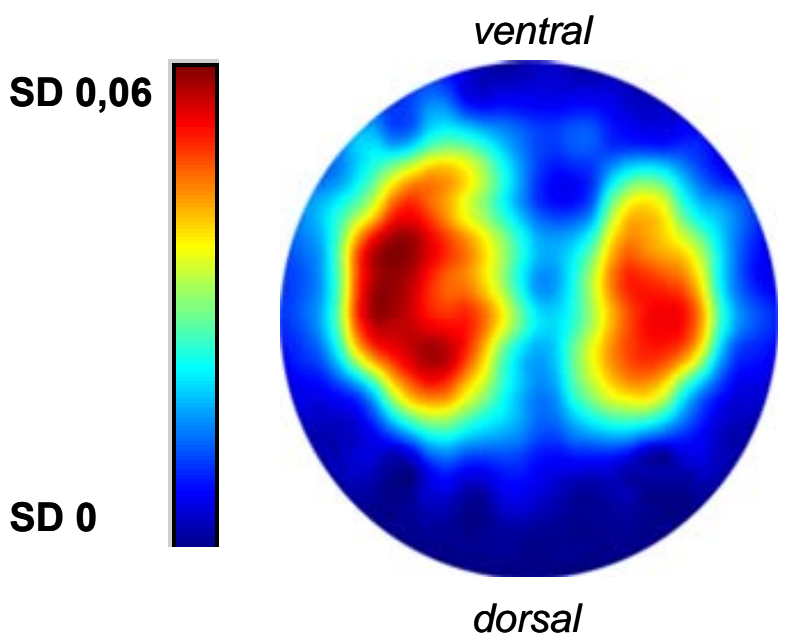

Abbildung 7: Funktionelle Impedanztomographie (f-EIT) eines Patienten. Die Variation der Impedanzänderung wird aus der Standardabweichung der regionalen Impedanzänderung einer EIT-Bildserie für jeden der 912 Bildpunkte berechnet. Regionen mit grosser regionaler Ventilation weisen eine grosse Variation (SD) auf und Regionen mit geringer regionaler Ventilation zeigen eine geringe Variation. 


\subsubsection{Regionale PV-Kurven}

Regionale PV-Kurven wurden bei den maschinell beatmeten Patienten während eines inspiratorischen Low-Flow-Manöver gemessen (Mankikian et al. 1983). Das Manöver wurde von einem Beatmungsgerät (Evita 4, Dräger AG, Lübeck, Germany) durchgeführt, dass von einem Laptop mit einer speziellen Software gesteuert wurde (Evita4Lab, Dräger AG, Lübeck, Germany). Aus Sicherheitsgründen wurde der Atemwegsdruck bei 45 mbar limitiert. Ein inspiratorisches Low-Flow-Manöver wurde gewählt, da hierbei die Atemwegswiderstände wegen des sehr geringen Gasflusses nur einen geringen Beitrag zur Atemmechanik leisten, so dass angenommen werden kann, dass der regionale Druck gleich dem Atemwegsdruck ist. Der Atemwegsdruck wurde mit einem Druckaufnehmer über eine Kapillare direkt am Endotrachealtubus gemessen (Druckwandler AP, SI-special instruments GmbH, Nördlingen, Germany). Aus den regionalen Impedanzänderungen, die proportional zum regionalen Luftgehalt sind und dem Atemwegsdruck wurden je Patient bis zu 912 regionale Druck-Volumen-Kurven gemessen. Aus diesen Druck-Volumen-Kurven wurden die unteren Inflektionspunkte und oberen Inflektionspunkte als charakteristische Kurvenpunkte eines sigmoiden Kurvenverlaufes durch Anpassung an eine sigmoide Formel berechnet (s. Abbildung 8 Seite 21) (Venegas et al. 1998). 


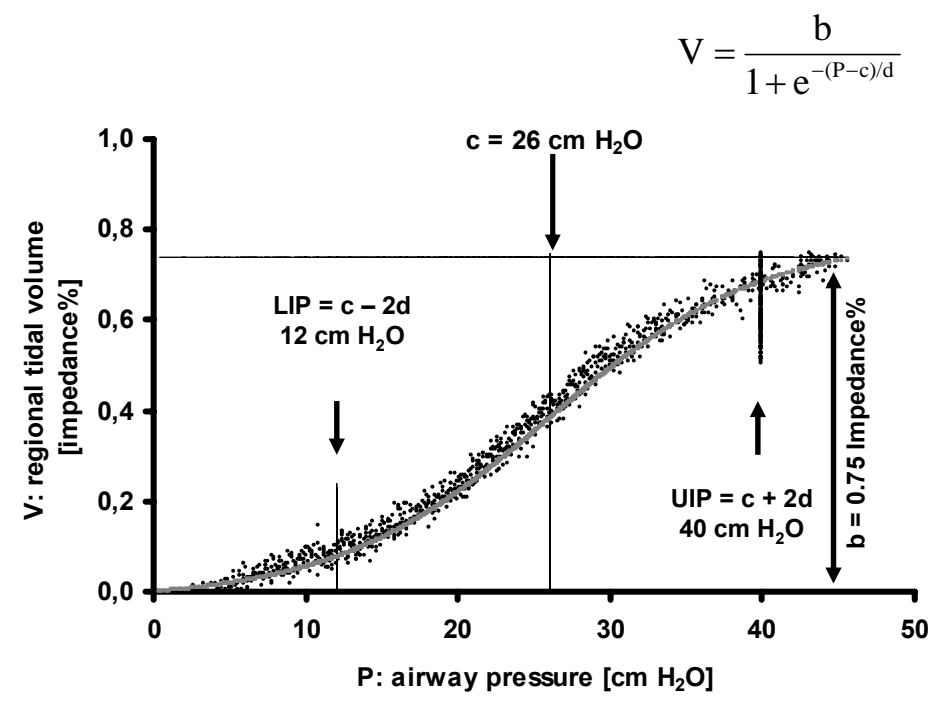

Abbildung 8: Berechnung der unteren (LIP) und oberen (UIP) Inflektionspunkte einer sigmoiden Druck-Volumen-Kurve durch Anpassung an die Gleichung $\mathrm{V}=\mathrm{b} /\left(1+\mathrm{e}^{-(\mathrm{P}-\mathrm{c}) / \mathrm{d}}\right)$ (Venegas et al. 1998).

\subsection{Pneumotachographie}

Die Messung des Gasflusses erfolgt mit dem Verfahren der Pneumotachographie (Fleisch 1925). Verwendet wurden beheizbare Pneumotachographen (Fleisch Nr. 2, Fa. Fleisch, Lausanne, Schweiz) und ein Differenzdruckabnehmer (Fa. Huba Control). Der Linearitätsbereich der verwendeten Pneumotachographen wird für den Gasfluß im Meßbereich von 0 bis 2,5 l/s mit $\pm 1 \%$ angegeben. Das Prinzip der Gasflussmessung beruht auf dem Druckabfall über zahlreichen lamellenartig angeordneten Kapillaren. Nach dem Gesetz von Hagen-Poiseuille ist der Druckabfall über jeder einzelnen Kapillare proportional zum Gasfluss. 


\subsection{Offener Stickstoffauswaschvorgang}

Zur Messung des end-exspiratorischen Lungenvolumens wurde ein offener Stickstoffauswaschvorgang in Anlehnung an Darling und Mitarbeiter (Darling et al. 1940) mit Modifikationen nach Wrigge und Mitarbeiter (Wrigge et al. 1998) durchgeführt. Das Prinzip des offenen Stickstoffauswaschvorganges basiert auf dem atemzugsweisen Auswasch eines Indikators (Stickstoff) mit reinem Sauerstoff. Hierzu wird während einer Einwaschphase mit Raumluft (Stickstoffgehalt $79 \%$ ) am Respirator auf reinen Sauerstoff umgestellt. Atemzugsweise wird die Menge des Indikators aus der Konzentration des Indikators durch Massenspektrometrie (MGA 1100 A, Fa. Perkin-Elmer, Pomona CA, USA) und dem Atemzugvolumen durch Pneumotachographie bestimmt. Das end-exspiratorische Lungenvolumen wird berechnet aus dem insgesamt ausgewaschenen Indikator und seiner initialen Konzentrationen (79\% Stickstoff).

\subsection{Statistik}

Die statistischen Berechnungen erfolgten mit einer Standardsoftware (STATISTIKA $^{\odot}$, Statsoft, Inc., Tulsa, OK, USA). Bei allen analytischen statistischen Verfahren wurde eine Irrtumswahrscheinlichkeit $\mathrm{p}<0,05$ für den $\alpha$-Fehler als signifikant betrachtet. Die Überprüfung der Ergebnisse auf Normalverteilung erfolgte mit dem Kolmogorov-Smirnov-Test. Bei Annahme der Normalverteilung wurden die Ergebnisse als Mittelwert \pm Standardabweichung angegeben. Die Prüfung auf signifikante Unterschiede erfolgte dann für verbundene Stichproben mit dem Student's-t-Test für verbundene und für unverbundene Stichproben mit dem Student's-t-Test für unverbundene 
Stichproben. Bei Ablehnung der Normalverteilung durch den KolmogorovSmirnov-Test wurden die Ergebnisse als Median und Bereich angegeben. Die Prüfung auf signifikante Unterschiede erfolgte in diesem Fall für verbundene Stichproben mittels Friedman-ANOVA oder dem Wilcoxon-Matched-Pairs-Test. Bei unverbundenen Stichproben kam eine Kruskal-Wallis-ANOVA oder ein Mann-Whitney-U-Test zur Anwendung.

Lineare Korrelationen wurden mit der Pearson Korrelation Analyse nach dem Prinzip der kleinsten Abstandsquadrate durchgeführt. Zusätzlich wurde eine Analyse nach „Bland and Altman“ (Bland und Altman 1986) durchgeführt. Der systematische Fehler wurde über den Bias aus den Mittelwerten der Differenzen zweier Methoden berechnet. Der zufällige Fehler wurde aus der Standardabweichung (SD) der mittleren Differenzen der zwei Methoden berechnet. Bias \pm 2 SD wurden als Grenzen der Übereinstimmung der zwei Methoden gewählt. 


\section{Ergebnisse}

\subsection{Studie I}

Inez Frerichs, José Hinz, Peter Herrmann, Gerhard Weisser, Günter Hahn, Taras Dudykevych, Michael Quintel, Gerhard Hellige (2002): Detection of local lung air content by electrical impedance tomography compared with electron beam CT. J Appl Physiol 93(2), 660-666

Insgesamt wurden 15.500 EBCT-Schichten und 98.000 EIT-Bilder an den

sechs mechanisch beatmeten Schweinen bei 15 Respiratoreinstellungen aufgezeichnet. Untersucht wurden Änderungen des Luftgehaltes in sechs regionsof-interest (ROI), die aus ventralen, medialen und dorsalen Regionen der rechten und linken Lunge ausgewählt wurden. Die Gesamtergebnisse zeigt die Abbildung 9 (Seite 25). Wir fanden für die Messung des Luftgehalts eine befriedigende Korrelation von EIT und EBCT. Die Korrelationskoeffizienten (R) schwankten von 0,75 in der ventral linken Lunge und 0,93 in der dorsal rechten Lunge, so dass wir die schlechteste Korrelation in den ventralen Lungenanteilen und die beste in den dorsalen Anteilen fanden. 


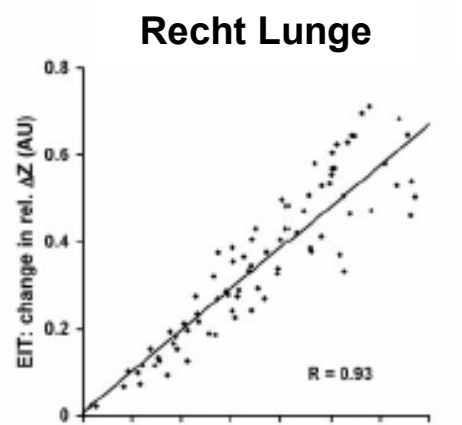

\section{Linke Lunge}

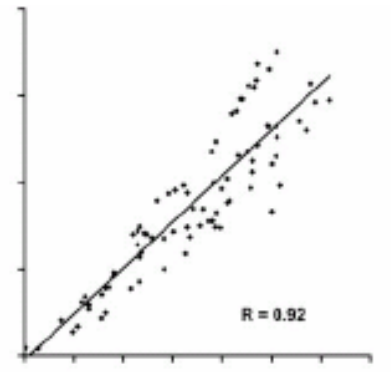

Dorsal
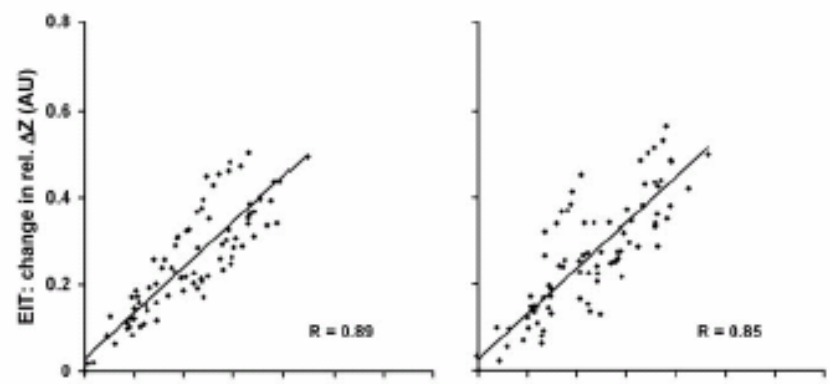

Mitte
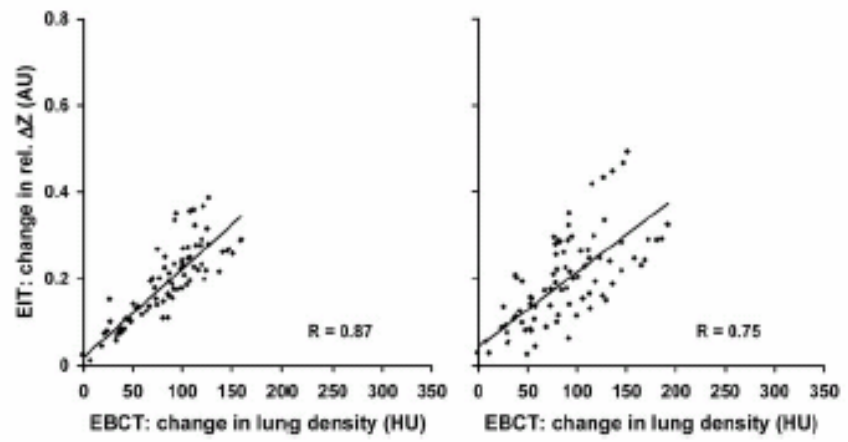

ventral

Abbildung 9: Vergleich der Luftgehaltsänderungen gemessen mit der elektrischen Impedanztomographie (EIT) und einem Elektronenstrahl-CT (EBCT) in den ausgewählten Regionen (Ventral, Mitte, Dorsal) der rechten und linken Lunge (Frerichs et al. 2002a). 


\subsection{Studie II:}

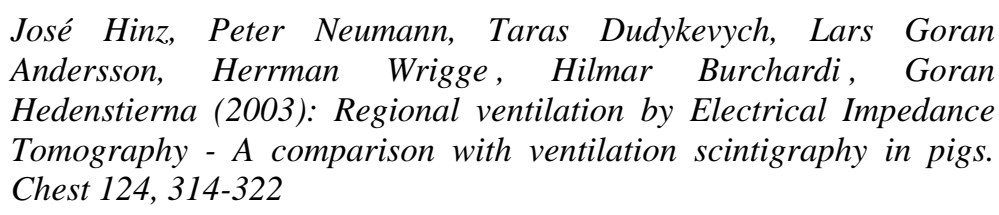

Wir schlossen zwölf Schweine mit ARDS in die Studie ein. Je vier Schweine wurden maschinell ohne Spontanatmung (PCV), maschinell unter Erhalt der Spontanatmung (APRV) und mit ausschliesslicher Spontanatmung (CPAP) beatmet. Der Oxigenierungsindex $\left(\mathrm{PaO}_{2} / \mathrm{F}_{\mathrm{i}} \mathrm{O}_{2}\right)$ als Mass der Lungenschädigung war im Mittel 176 mm Hg in der PCV Gruppe, 197 mm Hg in der APRV Gruppe und 212 mm Hg in der CPAP Gruppe. Wir fanden eine hohe lineare Korrelation der regionalen Ventilation zwischen EIT und Ventilationsszintigraphie (SPECT) $\left(\mathrm{Y}=82 \mathrm{x}+0,73 \mathrm{R}^{2}=0,92\right)$. Aus der BlandAltman-Analyse wird deutlich, dass EIT Regionen mit geringer Ventilation überschätzt und Regionen mit hoher Ventilation unterschätzt (s. Abbildung 10, Seite 27). Gleiche Befunde wurden erhoben bei einer Subgruppenanalyse des Beatmungsmodus (s. Abbildung 11, Seite 28 und Abbildung 12, Seite 29) und der Schwere des Lungenödems durch eine getrennte Analyse dorsaler und ventraler Lungenanteile (s. Abbildung 13, Seite 30). 

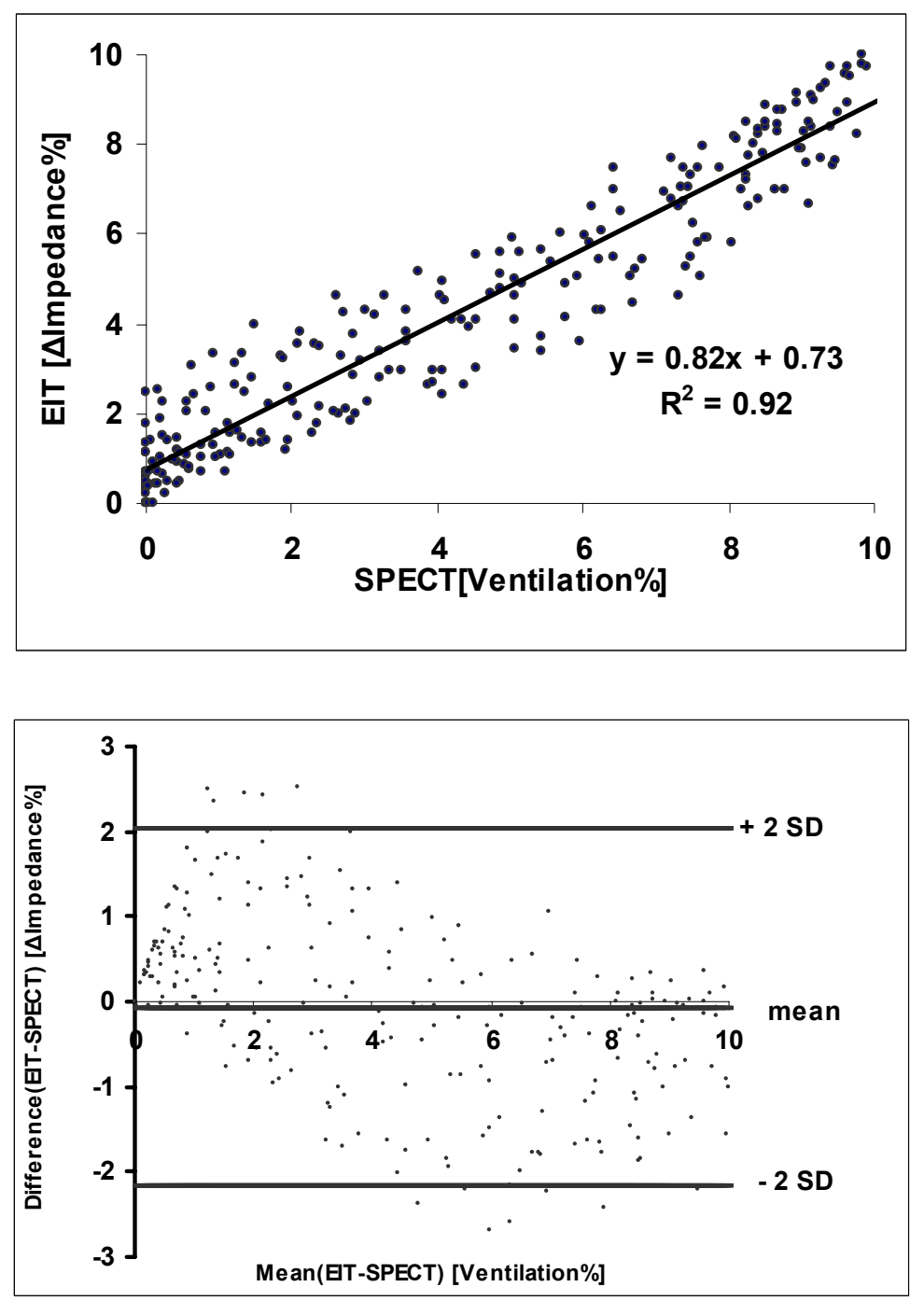

Abbildung 10: Lineare Korrelation und Bland-Altman-Analyse der regionalen Ventilation in 240 Regions-of-Interest einer Thoraxschicht zwölf maschinell beatmeter Schweine gemessen mit der elektrischen Impedanztomographie (EIT) und Ventilationsszintigraphie (SPECT). Ergebnisse als prozentualer Anteil der Ventilation an der Gesamtventilation in der untersuchten Thoraxschicht (Hinz et al. 2003b). 

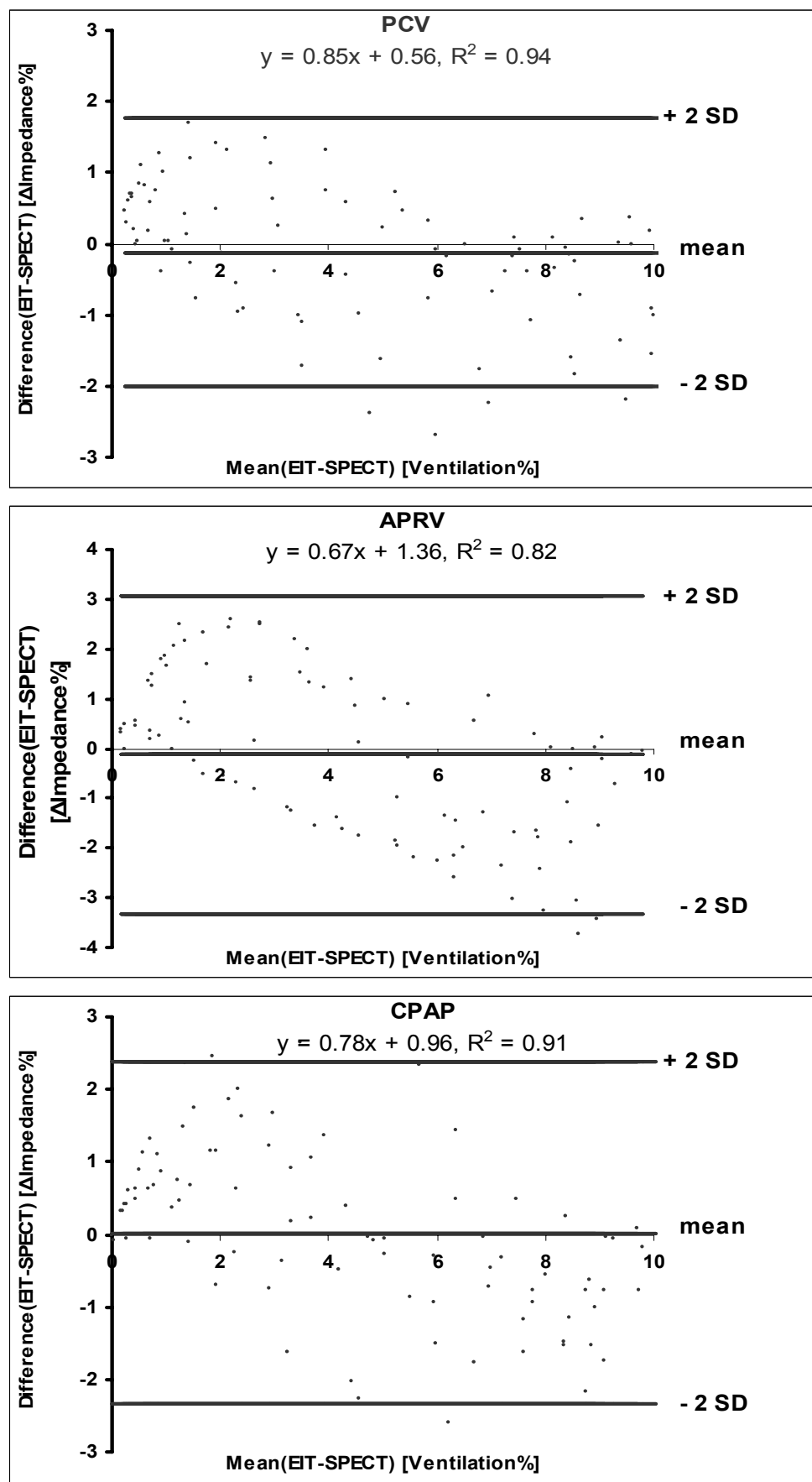

Abbildung 11: Lineare Korrelation und Bland-Altman-Analyse der regionalen Ventilation in 240 Regions-of-Interest einer Thoraxschicht zwölf machinell beatmeter Schweine gemessen mit der elektrischen Impedanztomographie (EIT) und Ventilationsszintigraphie (SPECT) in Abhängigkeit vom Beatmungsmodus Ergebnisse als prozentualer Anteil der Ventilation an der Gesamtventilation in der untersuchten Thoraxschicht. Je vier Schweine wurden maschinell ohne Spontanatmung (PCV), maschinell unter Erhalt der Spontanatmung (APRV) und mit ausschliesslicher Spontanatmung (CPAP) beatmet (Hinz et al. 2003b). 
SPECT
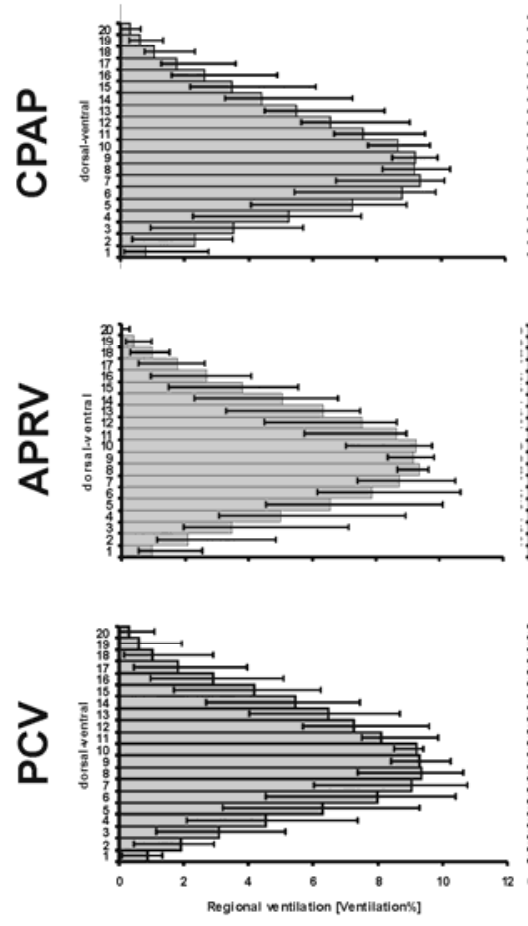
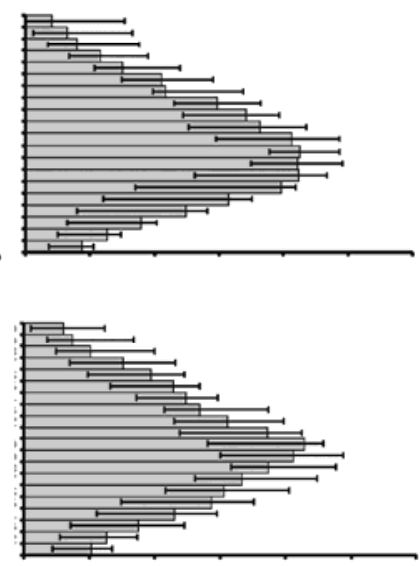

EIT

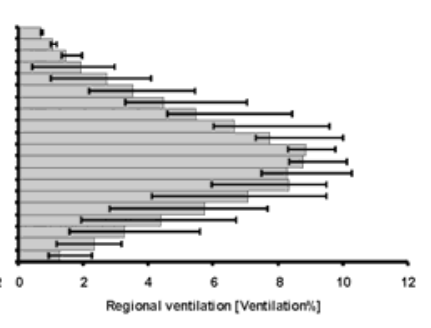

Abbildung 12: Vergleich der regionalen Ventilationverteilung in dorsaler zu ventraler Richtung einer Thoraxschicht bei verschiedenen Beatmungsmodi (PCV, APRV, CPAP). Ergebnisse als prozentualer Anteil der Ventilation an der Gesamtventilation in der untersuchten Thoraxschicht. Je vier Schweine wurden maschinell ohne Spontanatmung (PCV), maschinell unter Erhalt der Spontanatmung (APRV) und mit ausschliesslicher Spontanatmung (CPAP) beatmet (Hinz et al. 2003b). 

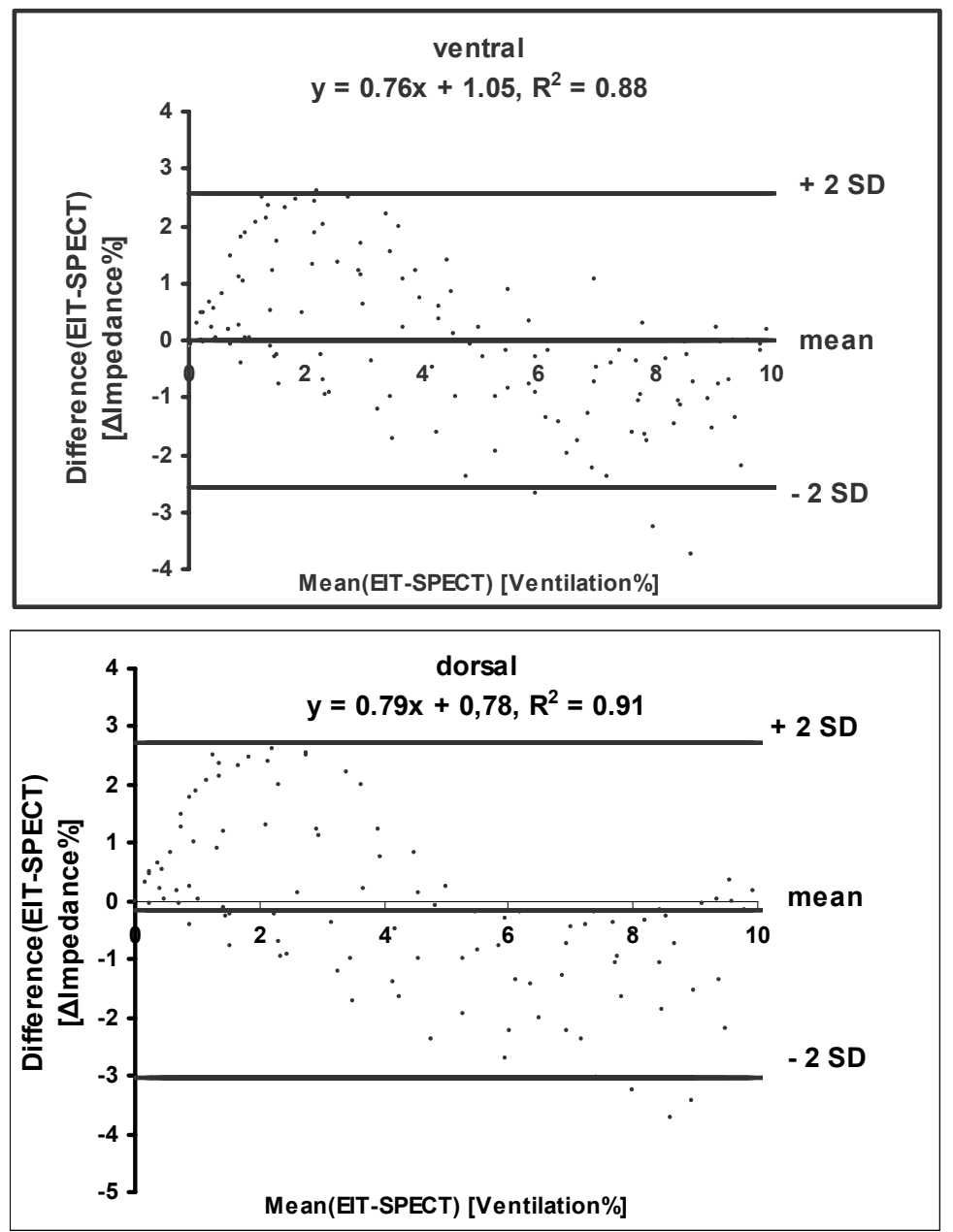

Abbildung 13: Lineare Korrelation und Bland-Altman-Analyse der regionalen Ventilation in 240 Regions-of-Interest einer Thoraxschicht zwölf machinell beatmeter Schweine gemessen mit der elektrischen Impedanztomographie (EIT) und Ventilationsszintigraphie (SPECT) in Abhängigkeit der Ausprägung des Lungenödems. Da bekannt ist, dass ein Lungenschaden durch zentralvenöse Ölsäureapplilkation eine schwerkraftabhängige Verteilung des Lungeschadens aufweist, wurde eine Analyse der Ventilation in den Subgruppen dorsaler Lungenregionen (dorsal) und ventraler Lungenregionen (ventral) durchgeführt. Ergebnisse als prozentualer Anteil der Ventilation an der Gesamtventilation in der untersuchten Thoraxschicht (Hinz et al. 2003b). 


\subsection{Studie III:}

José Hinz, Günter Hahn, Peter Neumann P, Michael Sydow, Peter Mohrenweiser, Gerhard Hellige, Hilmar Burchardi (2003): Endexpiratory lung impedance change enables bedside monitoring of endexpiratory lung volume change. Intensive Care Med 1, 37-43

Wir untersuchten die Änderung des end-exspiratorische Lungenvolumens (EELV) von zehn maschinell beatmeten Patienten mit EIT und einem offenen Stickstoffauswaschvorgang. Das end-exspiratorische Lungenvolumen der Patienten wurde durch stufenweise Änderung des PEEP von 0 mbar bis 15 mbar in 5 mbar Schritten variiert. Wir fanden bei 0 mbar PEEP ein EELV von $815 \mathrm{ml}$ bis $2002 \mathrm{ml}$ (Median $1.316 \mathrm{ml}$ ), bei 5 mbar PEEP von $940 \mathrm{ml}$ bis $2.281 \mathrm{ml}$ (Median $1.578 \mathrm{ml}$ ), bei 10 mbar PEEP 1.080-2.841 ml (Median $1944 \mathrm{ml}$ ) und bei 15 mbar PEEP ein EELV von 1.546-3.548 ml (Median 2379 ml). Die endexspiratorische Impedanzänderung variierte im Vergleich zur Referenz (0 mbar PEEP) bei 5 mbar PEEP von 0,00 bis 0,04 (Median 0,01), bei 10 mbar PEEP von 0,02 bis 0,08 (Median 0,03) und bei 15 mbar PEEP von 0,05 bis 0,13 (Median 0,06) (s. Abbildung 14 Seite 32). Die Langzeitstabilität des EIT Signals zeigte nach 1, 10, 20, 30, und 40 Minuten eine Variation von 1,5 bis 6,1\% (Median 3,1\%). Der Vergleich der mit den beiden Methoden gemessenen Lungenvolumenänderungen zeigte eine hohe lineare Korrelation (Abbildung 15, Seite 33). 

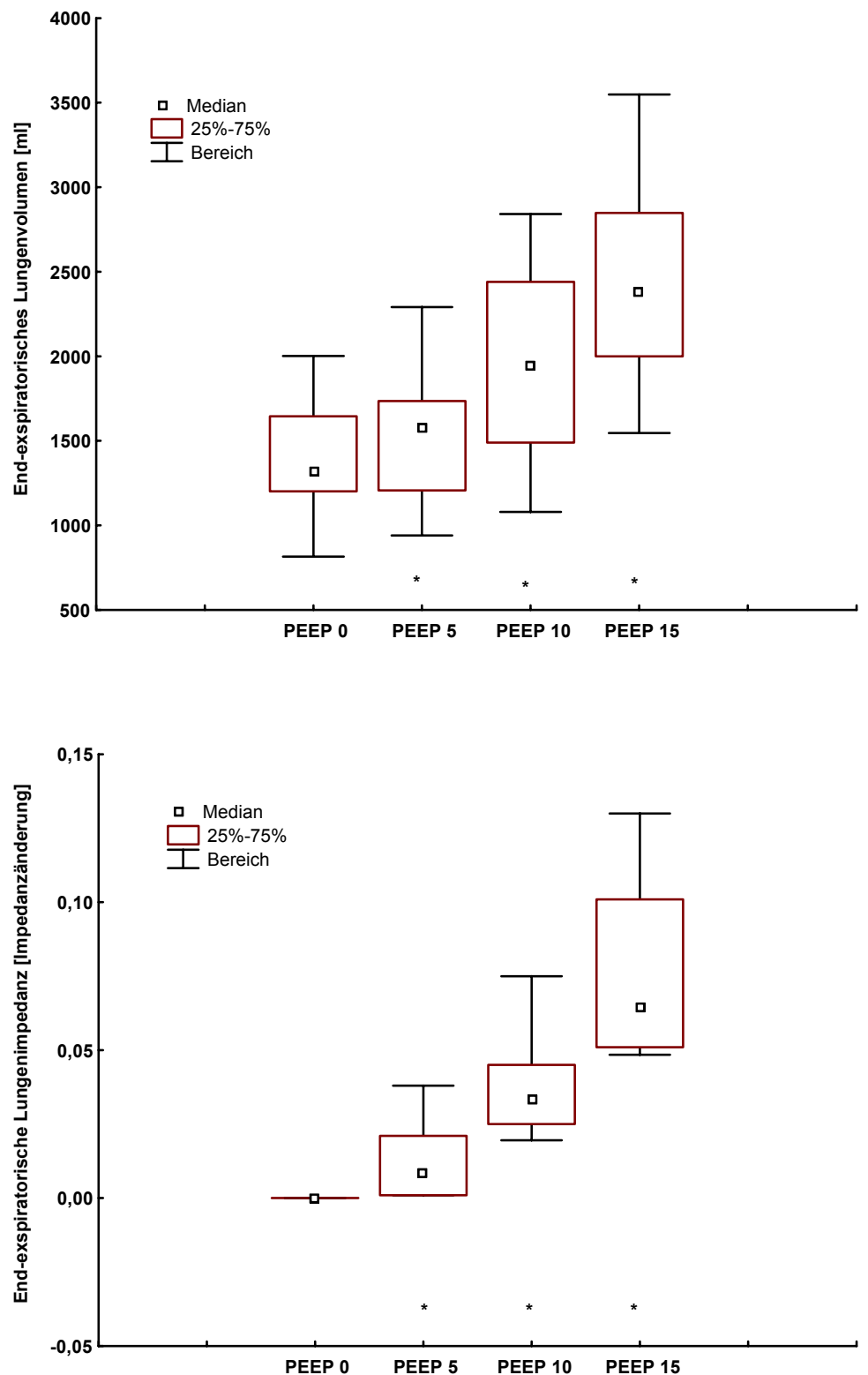

Abbildung 14: End-exspiratorisches Lungenvolumen und endexspiratorische Lungenimpedanz (ELIC) von 10 maschinell beatmeten Patienten bei unterschiedlichen PEEP * $p<0.05 \mathrm{im}$ Vergleich zur Referenz bei 0 mbar PEEP (Hinz et al. 2003a). 

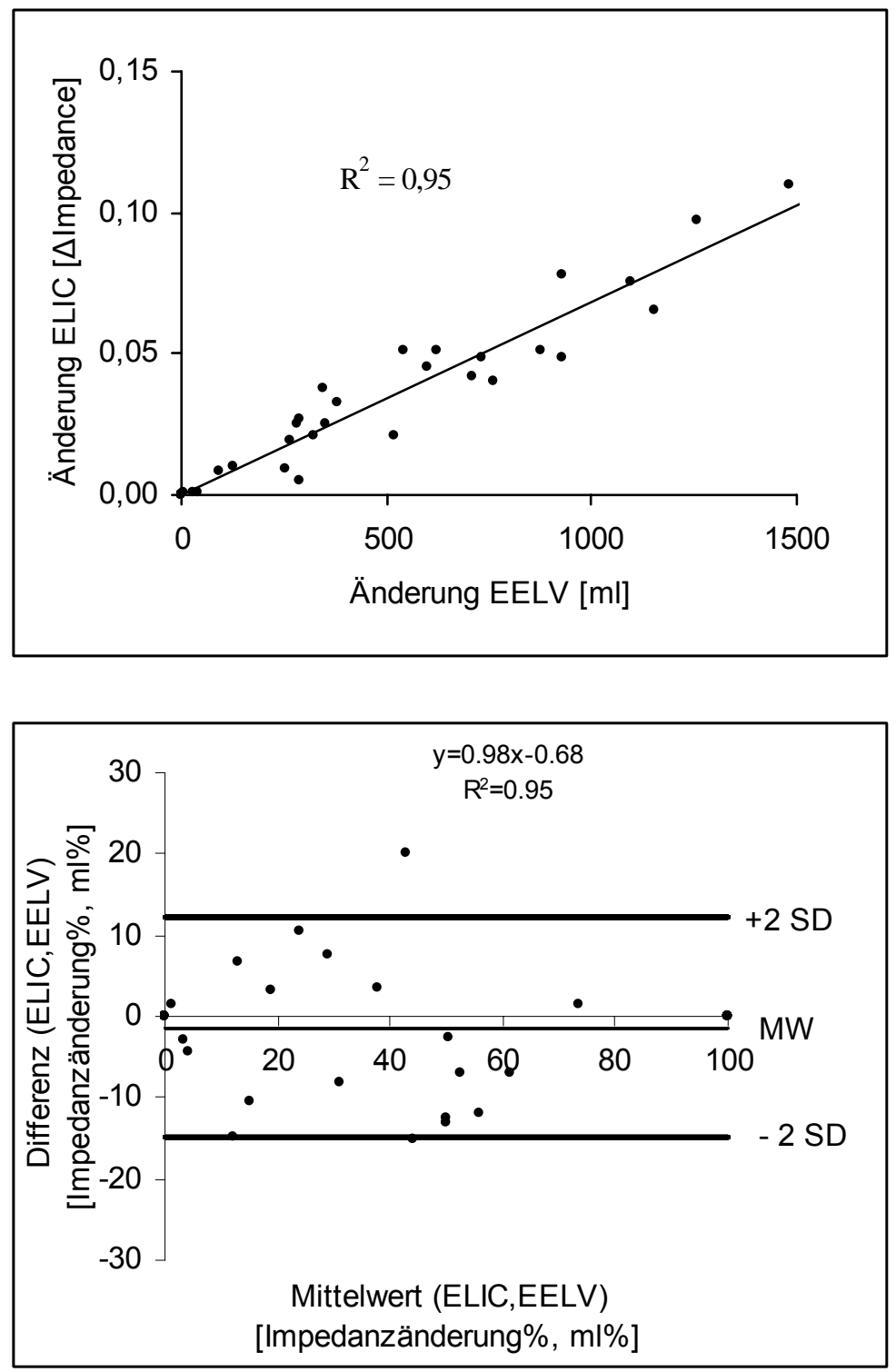

Abbildung 15: Lineare Korrelation und Bland-Altman-Analyse der Änderung des end-exspiratorischen Lungenvolumens von zehn maschinell beatmeten Patienten gemessen mit der elektrischen Impedanztomographie (ELIC) und einem offenen Stickstoffauswaschvorgang (EELV) (Hinz et al. 2003a). 


\subsection{Studie IV:}

José Hinz, Peter Mohrenweiser, Peter Neumann, Günter Hahn, Michael Sydow, Gerhard Hellige and Hilmar Burchardi (2005): Effects of positive end expiratory pressure on regional ventilation in mechanically ventilated patients. Eur J Anaesth 22(11), 817-825

Wir fanden eine Zunahme des end-exspiratorischen Lungenvolumens durch schrittweise PEEP Erhöhung im Bereich von $1.316 \mathrm{ml}$ bei 0 mbar PEEP auf $2.561 \mathrm{ml}$ bei 15 mbar PEEP. Dies entsprach einer Zunahme des EELV um 95\%. Nach Rückkehr auf 0 mbar PEEP war das EELV signifikant höher $(1.419 \mathrm{ml})$ als beim initialen 0 mbar PEEP. Die arterielle Sauerstoffsättigung und das arterielle Kohlendioxid blieben konstant bei allen PEEP. Der Oxigenierungsindex $\left(\mathrm{P}_{\mathrm{a}} \mathrm{O}_{2} / \mathrm{F}_{\mathrm{i}} \mathrm{O}_{2}\right)$ stieg von $215 \mathrm{mmHg}$ bei 0 mbar PEEP auf $286 \mathrm{~mm} \mathrm{Hg}$ bei 15 mbar PEEP. Nach anschliessender Rückkehr auf 0 mbar PEEP war der $\mathrm{P}_{\mathrm{a}} \mathrm{O}_{2} / \mathrm{F}_{\mathrm{i}} \mathrm{O}_{2}$ mit 240 mmHg signifikant höher als zu Beginn der Studie.

Die Anzahl der Regionen in der Gruppe “nicht-ventiliert” (Atelektasen, Thoraxwand und Mediastinum) nahm um $25 \%$ von 540 Regionen bei 0 mbar PEEP auf 406 Regionen bei 15 mbar PEEP ab. Die Regionen in der Gruppe “schlecht-ventiliert” nahm um 22 \% von 318 Regionen bei 0 mbar PEEP auf 360 Regionen bei 15 mbar PEEP zu. Die Regionen “moderat-ventiliert” nahm um das 2,5 fache von 40 Regionen bei 0 mbar PEEP auf 100 Regionen bei 15 mbar PEEP zu. Und die Anzahl der Regionen "gut-ventiliert” stieg signifikant von 0 bei 0 mbar PEEP auf 34 Regionen bei 15 mbar PEEP. Die Ergebnisse sind zusammengefasst in der Abbildung 16 (Seite 35). Wir fanden eine überwiegende Zunahme der Ventilation überwiegend in den dorsalen Lungenabschnitten (Abbildung 17, Seite 36). 

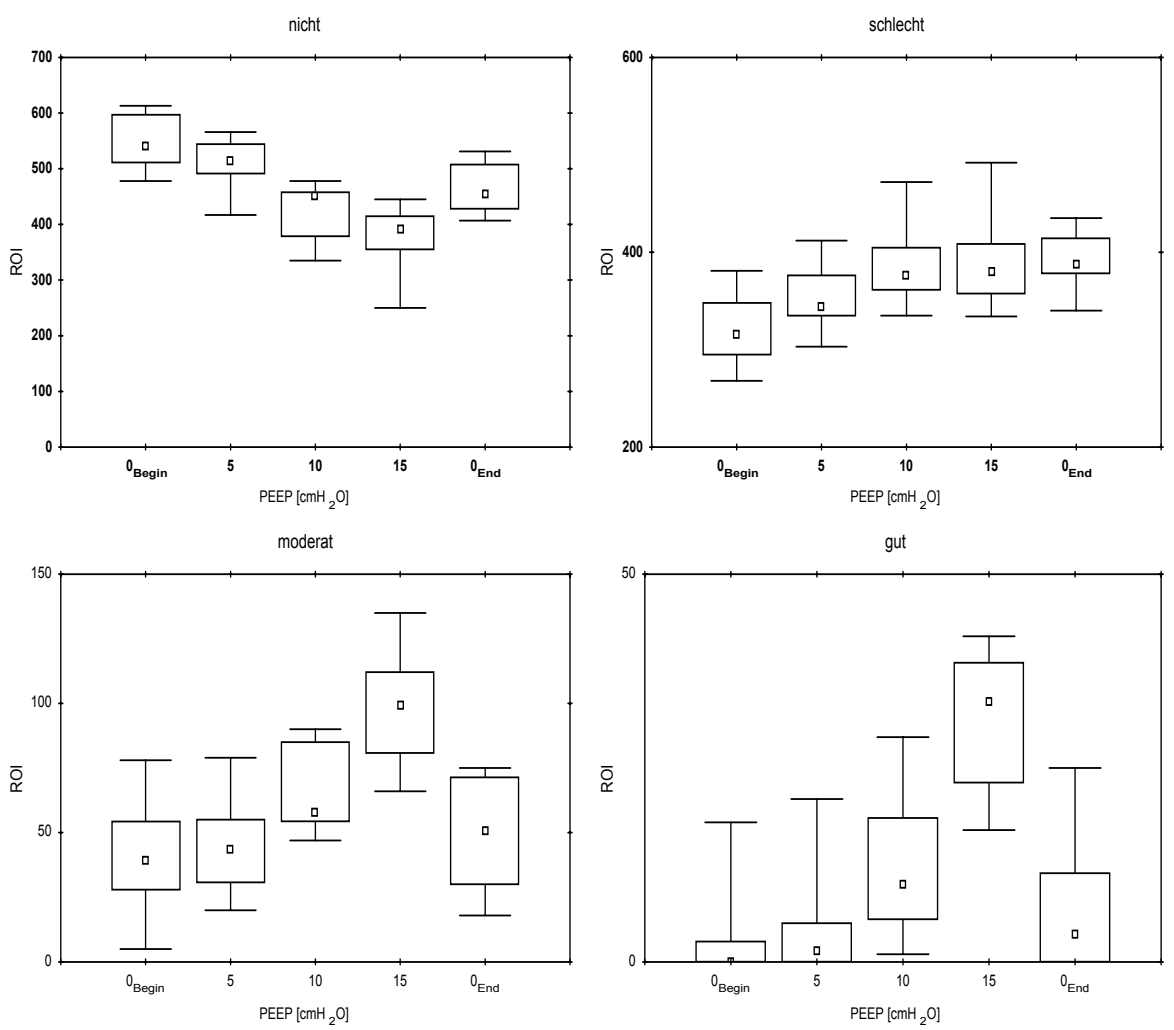

Abbildung 16: Anzahl der Regionen in den Ventilationsgruppen (nicht, schlecht, moderat, gut) von acht maschinell beatmeten Patienten mit akutem Lungenversagen bei verschiedenen PEEP (Hinz et al. 2005). 


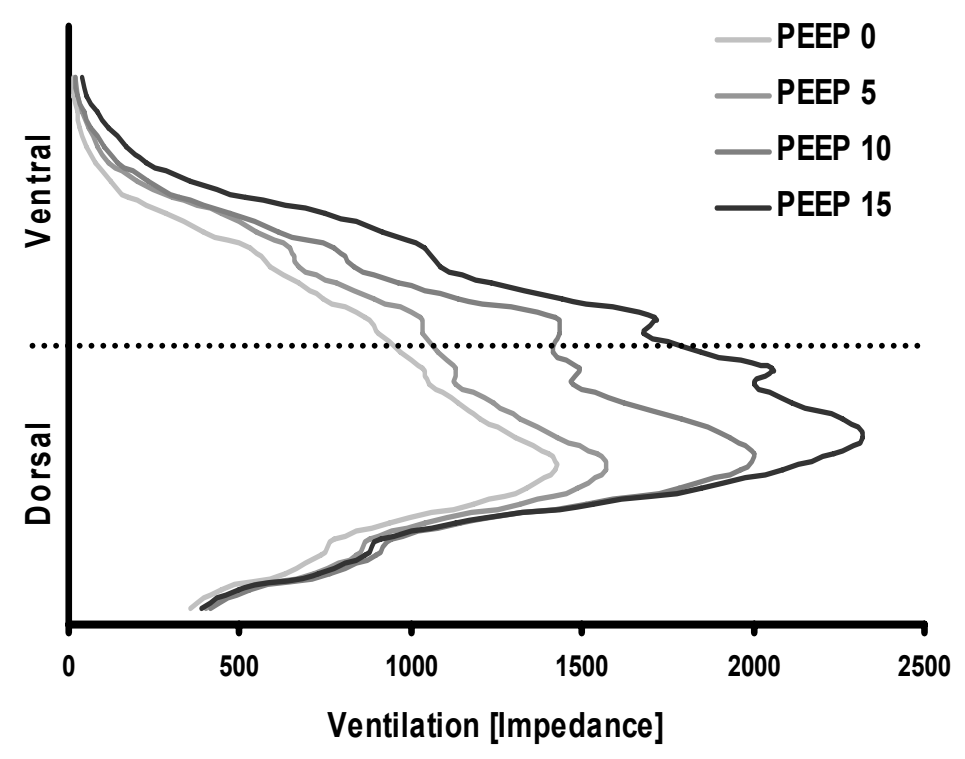

Abbildung 17: Summe der regionalen Ventilation (Impedance) von acht maschinell beatmeten Patienten mit akutem Lungenversagen bei verschiedenen PEEPs in dorso-ventraler Richtung (Hinz et al. 2005).

\subsection{Studie V}

José Hinz, Onnen Moerer, Peter Neumann, Taras Dudykevych, Inez Frerichs, Gerhard Hellige, Michael Quintel (2006): Regional pulmonary pressure volume curves in mechanically ventilated patients with acute respiratory failure measured by Electrical Impedance Tomography. Acta Anaesthesiol Scand 50, 331-339

Wir untersuchten neun maschinell beatmete Patienten mit akutem Lungenversagen. Der mittlere Lung injury score nach Murray (Murray et al. 1988) war 2,0 und der Oxigenierungsindex $\mathrm{P}_{\mathrm{a}} \mathrm{O}_{2} / \mathrm{F}_{\mathrm{I}} \mathrm{O}_{2}$ war 143-298 (Median 260) mmHg. Die Compliance streute von 30 - 82 (Median 51) ml/cm $\mathrm{H}_{2} \mathrm{O}$. Während des Low-Flow-Manövers, das bei einem Atemwegsdruck von 45 mbar aus 
Sicherheitsgründen unterbrochen wurde, beobachteten wir keine signifikanten Nebenwirkungen, wie Blutdruck- oder Sättigungsabfälle.

Die während des Low-Flow-Manövers inspirierten Tidalvolumina aller Patienten korrelierten signifikant mit der Impedanzänderung innerhalb der untersuchten EIT-Schicht $\left(y=1.06 x-7.4, \quad r^{2}=0.96\right)$. Aufgrund eines neuen Algorithmus zur Detektion von Lungenregionen innerhalb des f-EIT-Bildes variierte der Grenzwert der relativen Impedanzänderung von 0.01-0.10 (0.02). Dies entsprach 2,8\%-8,9\% (3,7\%) der maximal beobachteten relativen Impedanzänderung. In früheren Studien wurde eine Impedanzänderung oberhalb $10 \%$ der Gesamtimpedanzänderung als lungenspezifisch gewertet. Der neue Algorithmus wurde möglich durch den Einsatz eines neuen EIT-Gerätes mit verbessertem Signal-Rausch-Verhältnis (Hahn et al. 2001). Die Anzahl der untersuchten Lungenregionen innerhalb des f-EIT-Bildes variierte deshalb von 445-682 (Median 573) Lungenregionen aus insgesamt 912 möglichen f-EIT Regionen. Somit entfielen $63 \%$ des f-EIT-Bildes auf ausgewertete Lungenregionen.

Wir berechneten insgesamt neun konventionelle PV-Kurven, neun globale EIT-PV-Kurven innerhalb der untersuchten Elektrodenebene und 4.512 regionale EIT-PV-Kurven. Diese 4.530 PV-Kurven wurden mit hoher Genauigkeit an die sigmoide Modellgleichung angepasst ( $\mathrm{r}^{2}$ 0.93-0.98 (Median 0.95). Aus den globalen PV-Kurven der konventionellen Messungen und EIT-PV-Kurven innerhalb der Elektrodenebene ( $\left.\mathrm{PV}_{\text {Spirometry }}, \mathrm{PV}_{\text {EITglobal }}\right)$ konnten bei acht von neun Patienten untere Inflektionspunkte (LIP) berechnet werden (Konventionell: 3-11 (Median 8) mbar; EIT-PV-Kurven: 4-12 (Median 7) mbar), die eine hohe lineare 
Korrelation zeigten $\left(y=1.05 x+0.03, r^{2}=0.93\right)$. Jedoch fanden wir in den regionalen Messungen eine ausgeprägte Heterogenität der Inflektionspunkte. LIP konnten in 54-264 (median 180) Regionen gefunden werden, die von 2-26 (Median 8) mbar variierten. Der Medianwert der LIP aus den regionalen Messungen war vergleichbar mit den globalen LIP.

In den globalen Messungen wurden obere Inflektionspunkte (UIP) bei drei Patienten gefunden (Konventionell: 31-39 (Median 33) mbar; EIT PV Kurven: 30-40 (Median 34 mbar). Wir fanden ebenfalls eine enge lineare Korrelation der UIP aus diesen Messungen $\left(y=1.19 x-6.08, R^{2}=0.97\right)$ und eine deutliche Heterogenität der regionalen UIP (14-44 (Median 36) mbar in 149 - 324 (193) Regionen) und einen vergleichbaren Medianwert der regionalen und globalen UIP. Alle Ergebnisse sind in Abbildung 18 (Seite 39) zusammengefasst. Desweiteren fanden wir einen Einfluss der Schwerkraft auf die Verteilung der LIP und UIP (s. Abbildung 19 Seite 40). 
LIP

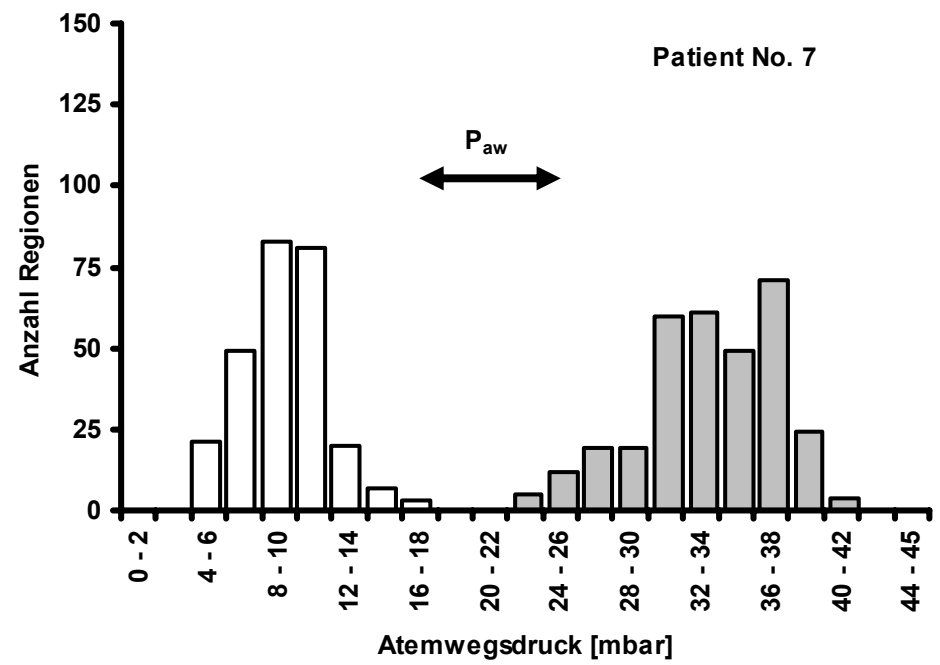

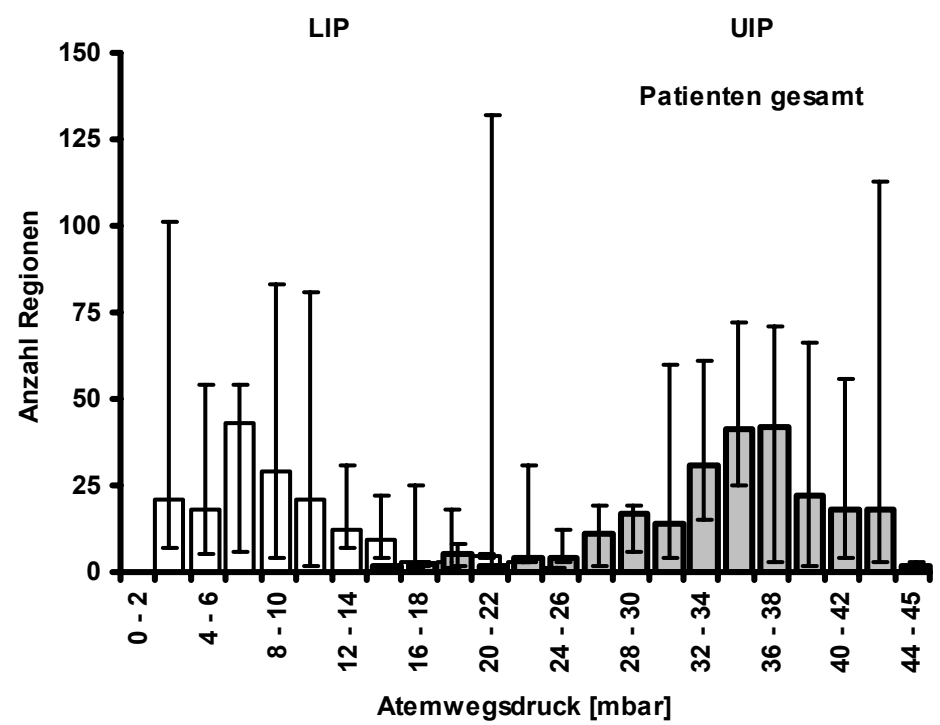

Abbildung 18: Regionale untere (LIP) und obere Inflektionspunkte (UIP) eines maschinell beatmeten Patienten (Patient No. 7) und aller untersuchter Patienten (Patienten gesamt) in einer transversalen Thoraxebene gemessen mit der elektrischen Impedanztomographie. Anhand der histographischen Verteilung (Patient 7) lässt sich möglicherweise der Atemwegsdruck so wählen, dass endexspiratorischer Kollaps und end-inspiratorische Überblähung in der Mehrzahl der Regionen vermieden werden (Hinz et al. 2006). 

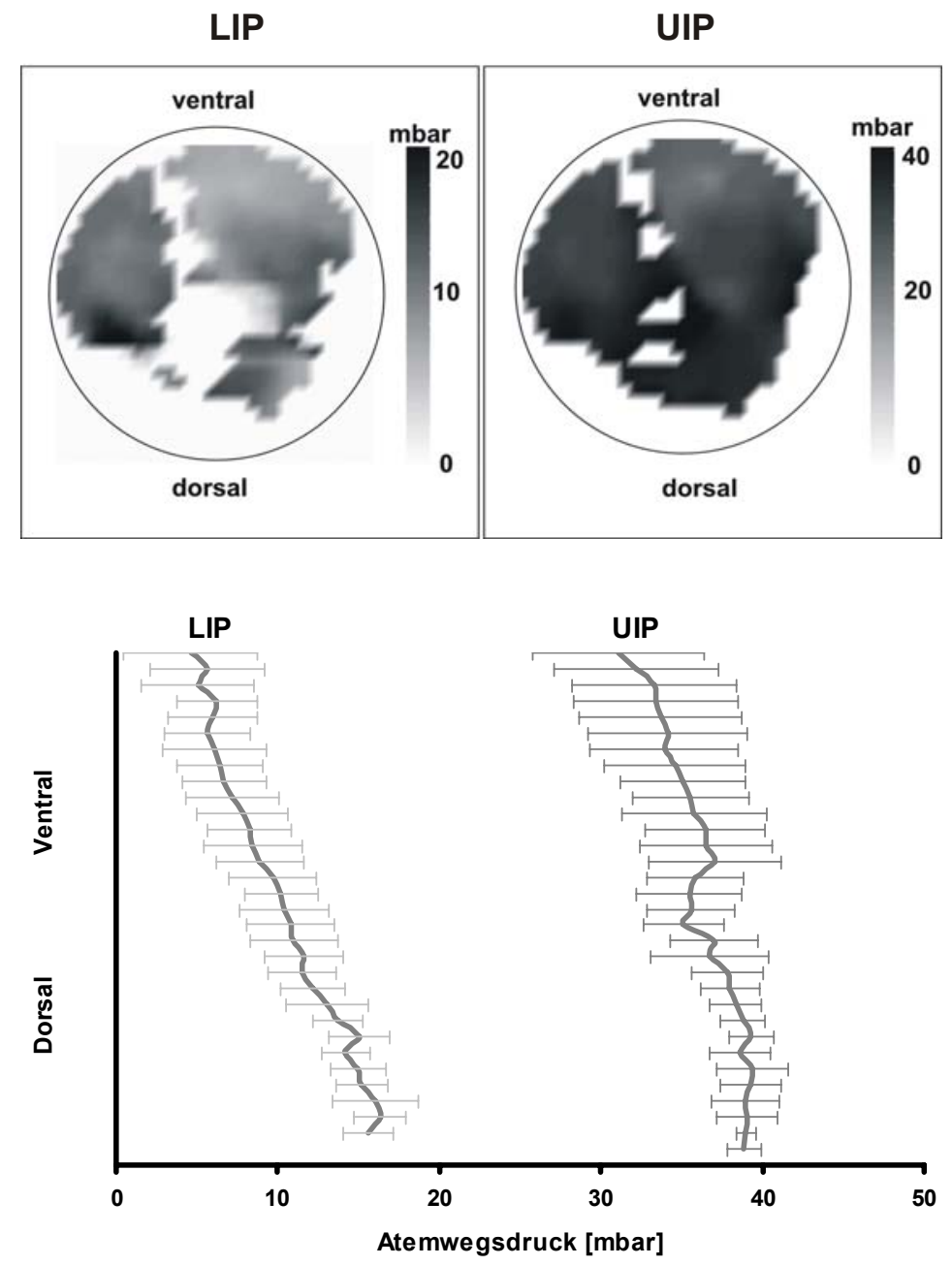

Abbildung 19: Regionale untere (LIP) und obere Inflektionspunkte (UIP) eines Patienten (oben) und aller untersuchten Patienten (unten) in einer transversalen Thoraxebene in dorso-ventraler Richtung (Hinz et al. 2006). (Werte oben als Absolutwerte unten als Mittelwert und Standardabweichung) 


\section{Diskussion}

Die maschinelle Beatmung schädigt bei inadäquaten Beatmungsdrucken sekundär die Lunge. Es konnte gezeigt werden, dass eine Beatmung deren Beatmungsdrucke durch globale PV-Kurven eingestellt wird die Prognose von Patienten mit akutem Lungenversagen günstig beeinflusst (Amato et al. 1998, Gattinoni et al. 1984, The Acute Respiratory Distress Syndrome Network 2000). Druck-Volumen-Kurven der gesamten Lunge sind jedoch nicht repräsentativ für alle Lungenregionen, da sich die Atemmechanik regional unterscheidet. Deshalb wurde empfohlen bei Respiratoreinstellung regionale Inhomogenitäten in die Überlegungen mit einzubeziehen. Da Atelektasen innerhalb von Sekunden auftreten können (Neumann et al. 1998a, Rothen et al. 1999), sind Techniken wünschenswert, die die regionale Funktion der Lunge kontinuierlich, bettseitig und nicht invasiv überwachen (Rouby et al. 2002). Die derzeitigen Untersuchungsmethoden Computertomographie und Ventilationsszintigraphie sind im Gegensatz zu der elektrischen Impedanztomographie hierzu nicht geeignet. Ziel dieser Habilitationsarbeit war der Vergleich der elektrischen Impedanztomographie, die diese Voraussetzungen erfüllt, mit klinisch etablierten Verfahren der Luftgehalts- und Ventilationsmessung und die Einführung von Parametern zur Messung der regionalen Ventilation.

1) Computertomographie als Referenzmethode der regionalen Luftgehaltsmessung (Studie I),

2) Ventilationsszintigraphie als Referenzmethode der regionalen Ventilation (Studie II)

3) Offener Stickstoffauswaschvorgang als Referenzmethode des end-exspiratorischen Lungenvolumens (Studie III). 
4) Inaugurierung bettseitiger Parameter zur Überwachung der regionalen Ventilation (Studie IV und V).

Die Ergebnisse der Studie I bestätigen, dass die EIT regionale Luftgehaltsänderungen der Lunge und deren Inhomogenität in ventralen, medialen und dorsalen Regionen beider Lungen messen kann. Die Unterschiede beruhen auf den Unterschieden der Messtechniken zwischen EIT und CT und sind in Bezug auf den Vergleich nicht unkritisch. EIT misst die elektrischen Eigenschaften des Lungengewebes und hat eine zeitliche Auflösung von 45 EITBilder $\cdot \mathrm{s}^{-1}$. Dies ist somit im Vergleich $\mathrm{zu}$ möglichen $4 \mathrm{CT}$-Bilder $\cdot \mathrm{s}^{-1}$ deutlich höher. Jedoch ist die örtliche Auflösung der EIT mit 8\% des Thoraxdurchmessers, so dass Volumina zwischen $9 \mathrm{ml}$ und $29 \mathrm{ml}$ untersucht werden können, geringer als die des CT (Brown und Barber 1987, Hahn et al. 1998). Die EIT ist deshalb geeigneter zur Messung funktioneller Änderungen der Lunge. Das CT hingegen misst die Gewebedichte und ist damit geeigneter zur Messung anatomischer und morphologischer Strukturen. Da bei unserer Untersuchung die Änderung des Luftgehalts zwischen end-exspiratorischen und end-inspiratorischen Status während ununterbrochener maschineller Beatmung berechnet wurde, ist die deutlich unterschiedliche zeitliche Auflösung in Bezug auf die untersuchte Ventilationsamplitude beim CT ungenauer als beim EIT. Bei einer Inspirationszeit von zwei Sekunden (Respiratorfrequenz 10·Minute-1, I:E=1:1,3) werden mit dem CT lediglich 8 CT-Bilder und mit der EIT 26 EIT-Bilder untersucht, so dass die end-inspiratorischen und end-exspiratorischen Respiratorphasen mit der EIT wegen der höheren zeitlichen Auflösung exakter detektiert werden. Ein weiterer Unterschied zwischen EIT und CT besteht in der 
Ausdehnung und Form der untersuchten Schicht. Während eine untersuchte CTSchicht eine nahezu gleichmässige Schichtbreite aufweist, fliesst der elektrische Strom der EIT entlang der Äquipotential-Linien, so dass auch Lungengewebe im Randbereich der durch die Elektroden definierten transversalen Thoraxschicht untersucht werden (Rabbani und Kabir 1991). Aus diesem Grund wurde mit dem CT eine multi-slice Untersuchung mit insgesamt vier CT-Schichten durchgeführt, um eine $4 \mathrm{~cm}$ CT-Schicht zu untersuchen. Die Ergebnisse der Studie zeigen beim Vergleich der regionalen Ventilation von EIT und CT eine schlechtere Korrelation in den mittleren und ventralen Lungenregionen. Hierfür sind vermutlich zwei Effekte verantwortlich. Bei den auf dem Rücken liegenden Tieren findet eine Bewegung des Thorax in ventraler Richtung statt. Der Bildrekonstruktionsalgorithmus berücksichtigt im Gegensatz zum CT keine Bewegungen des untersuchten Körpers, so dass das CT die ventralen Thoraxexkursionen im Gegensatz zum EIT mit untersucht. Ein weiterer Grund liegt in Impedanzartefakten durch den pulsatilen Blutstrom des ventral liegenden Herzens (Faes et al. 1999, Frerichs et al. 2002b, Vonk et al. 1997). Trotz der erwähnten Unterschiede sind sowohl die EIT (Nopp et al. 1993) als auch das CT (Drummond 1998) geeignet Unterschiede im regionalen Lungenvolumen zu messen. Die Hauptaussage der Studie I liegt in der trotz der beschriebenen Limitierungen guten Übereinstimmung von EIT und CT zur regionalen Luftgehaltsmessung bei gesunden Lungen.

Die Studie II zeigt im Modell eines akuten Lungenversagens, dass eine Überwachung der regionalen Ventilation und deren Verteilung bettseitig durchgeführt werden kann. Bereits früher wurden Vergleichsuntersuchungen der 
EIT mit der Ventilationsszintigraphie (SPECT) durchgeführt und eine gute Korrelation der EIT mit SPECT gefunden (Kunst et al. 1998). In dieser Studie wurde das Verhältnis der Ventilation der rechten und linken Lunge als „regionsof-interests“ (ROI) mit der EIT und SPECT verglichen. Der Unterschied in unserer Studie lag in der höheren örtlichen Auflösung. Während Kunst und Mitarbeiter zwei ROIs verglichen und wegen des schlechten Signal-RauschAbstandes jeweils 10 EIT-Bilder mitteln mussten, um ein EIT-Bild zu berechnen, untersuchten wir 20 Regionen, die von dorsal nach ventral verliefen. Die erhöhte Anzahl der ROIs war möglich, da das in der Abteilung Anästhesiologische Forschung entwickelte und von uns verwendete EIT-Gerät ein deutlich verbessertes Signal-Rausch-Verhältnis aufwies (Hahn et al. 2001), so dass 912 ROIs mit 45 EIT-Bildern.s ${ }^{-1}$ gemessen werden konnte. Der Auswertealgorithmus des SPECT liess jedoch nur 20 dorso-ventrale Schichten als ROI zu. Wir beobachteten eine gute lineare Korrelation zwischen EIT und SPECT unabhängig davon, ob es sich um Spontanatmung oder kontrollierte Beatmung handelt (s. Abbildung 11, Seite28). Wir fanden auch keinen Unterschied in der Korrelation in Abhängigkeit von der Schwere des Lungenschadens (s. Abbildung 13, Seite 30), der Compliance oder des extravaskulären Lungenwassers. Wir beobachteten jedoch, das EIT die regionale Ventilation im Vergleich zu SPECT in den gut ventilierten Regionen unterschätzt. Wohingegen EIT die regionale Ventilation in den schlecht ventilierten Regionen überschätzt. Ein Grund hierfür könnte in der Ablagerung des Indikators in den Atemwegen sein, da hierdurch eine erhöhte Ventilation in diesen Regionen vortäuscht wird. Ein weiterer Grund liegt vermutlich in den Besonderheiten der Bildrekonstruktion. In einem mit 
isotoner Kochsalzlösung gefüllten Gefäss als physiologischem Modell eines Thorax wurde der lineare Bereich des Bildrekonstruktionsalgorithmusses untersucht. Bis zu 20\% Impedanzänderung verhält sich der Algorithmus linear. Wir beobachteten Impedanzänderungen bis zu 25 \%, so dass die Fehlschätzungen darauf zurückzuführen sein können. Zusätzlich basiert der back-projectionAlgorithmus auf einigen Annahmen, die zum Teil bei Thoraxuntersuchungen und Lungenversagen verletzt werden. Das zu untersuchende Objekt muss zweidimensional und rund sein, die regionale Impedanz muss homogen sein und die Elektroden müssen äquidistant um das Objekt verteilt sein (Barber 1990). Die Limitierungen der zeitlichen und räumlichen Auflösung, die bereits in Studie I beim Vergleich mit dem CT besprochen wurden, gelten für den Vergleich der EIT mit SPECT ebenso. Die Hauptaussage der Studie II liegt in der trotz der beschriebenen Limitierungen guten Übereinstimmung von EIT und SPECT zur regionalen Ventilationsmessung bei Schweinen mit ARDS. Die Ergebnisse sind mit hoher Wahrscheinlichkeit auf den beatmeten Patienten mit akutem Lungenversagen oder ARDS übertragbar.

Das end-exspiratorische Lungenvolumen (EELV) ist ein Parameter zur Beurteilung der Lungenfunktion beatmeter Patienten, da es indirekt Informationen über Atelektasen oder mögliche Überblähung gibt (Dambrosio et al. 1997, Gattinoni et al. 1995, Hedenstierna 1993). Eine kontinuierliche Überwachung ist insbesondere von Bedeutung, da Atelektasen innerhalb von einigen Sekunden entstehen können (Neumann et al. 1998a, Rothen et al. 1999) und somit eine bettseitige Überwachung des EELV beispielsweise nach akzidenteller Diskonnektion vom Respirator oder nach endotrachealer Absaugung 
wünschenswert ist. Die Studie III wurde deshalb durchgeführt, um EELVÄnderungen mit EIT und dem offenen Stickstoffauswaschvorgang als Referenzverfahren miteinander zu vergleichen (Darling et al. 1940, Wrigge et al. 1998). Die Ergebnisse zeigen, dass eine Erhöhung des EELV eine Erhöhung des end-exspiratorischen Impedanzniveaus nach sich zieht und dass dieser Zusammenhang eine hohe lineare Korrelation aufweist. Jedoch wird bei kleinen EELV-Änderungen die Änderung von EIT überschätzt, während bei grossen EELV-Änderungen die Lungenvolumenänderungen von EIT unterschätzt werden. Der Einfluss des Bildrekonstruktionsalgorithmus auf die Impedanzberechnung wurde bereits oben erläutert. Ein weiterer Grund liegt darin, dass ein offener Stickstoffauswaschvorgang die Änderung des EELV der gesamten Lunge misst, wohingegen die EIT die Änderung in einer ca. 4 cm dicken Thoraxschicht untersucht. Beim akuten Lungenversagen liegen nicht nur regionale Inhomogenitäten in dorso-ventraler Richtung vor, sondern auch in cephalocaudaler Richtung (Puybasset et al. 2000, Wrigge et al. 2003), so dass die Ergebnisse hiervon beeinflusst werden können. Ein möglicher Ausweg aus diesem Problem könnten die Einfügung weiterer EIT-Untersuchungsebenen oder die Entwicklung eines dreidimensionalen EIT sein (Metherall et al. 1996). Zusätzlich muss angemerkt werden, dass ein offener Stickstoffauswaschvorgang auf dem vollständigen Auswasch des Indikators beruht. Lungenregionen mit langen Zeitkonstanten, wie man sie üblicherweise bei Patienten mit Lungenversagen findet, werden deshalb möglicherweise nicht vollständig vom Indikator ausgewaschen, so dass das EELV geringer ausfällt als die Ergebnisse, die auf der Messung der EIT beruhen. 
Studie IV wurde durchgeführt, um bettseitig auf der Intensivstation den Einfluss von PEEP auf die regionale Ventilation bei mechanisch beatmeten Patienten mit akutem Lungenversagen $\mathrm{zu}$ untersuchen Wir fanden eine Verbesserung des Gasaustausches bei schrittweiser Erhöhung des PEEP von 0 mbar auf 15 mbar. Die Verbesserung des Gasaustausches beruht zum Teil auf einer Zunahme des EELV, die auf einer Umverteilung nicht ventilierter Regionen hin zu gut ventilierten Regionen basiert. Die beobachtete verbesserte regionale Ventilation nach Änderung von 15 mbar PEEP auf 0 mbar PEEP im Vergleich zur regionalen Ventilation zu Beginn der Studie bei 0 mbar PEEP basiert vermutlich auf nachhaltiger Rekrutierung atelektatischer Regionen durch ein Studienprotokoll, dass einem „PEEP trial“ sehr ähnlich ist (Lim et al. 2003).

Ein methodisches Problem dieser Untersuchung basiert darauf, dass mit der EIT nur Untersuchungen in einer EIT-Ebene durchgeführt werden können und dass bereits wie oben erwähnt regionale Inhomogenitäten nicht nur in dorsoventraler sondern auch in cranio-caudaler Richtung gefunden wurden (Puybasset et al. 2000, Wrigge et al. 2003). Während der mechanischen Beatmung wandert zusätzlich die Lunge innerhalb der Untersuchungsebene nach caudal, so dass durch die EIT verschiedene Lungenregionen untersucht werden. Die Analyse einer einzigen EIT-Ebene wird damit die regionale Inhommogenität möglicherweise unterschätzen. Gleiche Vorbehalte wurden bereits früher bei Untersuchungen der Lunge mit CT oder SPECT aufgeworfen.

Ein f-EIT-Bild beinhaltet Regionen der Thoraxwand, des Mediastinum, Atelektasen und ventilierter Lungenregionen und basiert auf der Rekonstruktion von regionalen Impedanzänderungen durch einen back-projection-Algorithmus, 
die sowohl durch Veränderungen im Luftgehalt als auch durch den pusatilen Blutfluss generiert werden (Faes et al. 1999, Frerichs et al. 2002b, Wtorek und Polinski 2005). Die regionalen Impedanzänderungen weisen deshalb eine grosse interindividuelle Heterogenität auf, so dass nicht zwischen Thoraxwand und Atelektase unterschieden werden kann. Zusätzlich können Atelektasen im geringen Signal-Rauschverhältnis der verwendeten EIT Gerätes älterer Generation versteckt sein. Da in unserer untersuchung die nicht ventilierten Regionen sowohl aus Thoraxwand, Mediastinum und Atelektasen bestanden, wurde eine Abnahme der nicht ventilierten Regionen bei physiologischerweise konstantem Anteil von Thoraxwand und Mediastimun als Rekrutierung einer Atelektase gewertet. In der Studie IV wurde als Grenzwert von $10 \%$ der maximal beobachteten Impedanzänderung als Atelektase bewertet (Hahn et al. 1996). In der Studie V wurde ein EIT-Gerät neuerer Generation verwendet mit deutlich verbessertem Signal-Rausverhälniss, so dass neue Konzepte der Atelektasendetektion verwendet werden konnten.

Bei mechanisch beatmeten Patienten treten Atelektasen hauptsächlich in den dorsalen Lungenabschnitten aufgrund des hydrostatischen Druckes und der reduzierten Zwerchfellbeweglichkeit bei maschineller Beatmung ohne Spontanatmungsanteil auf (Hedenstierna et al. 1985). PEEP vermeidet den regionalen Kollaps dann, wenn der PEEP gleich oder grösser dem regionalen hydrostatischen Druck ist (Gattinoni et al. 1993). Diese Ergebnisse wurden durch unsere Studie bestätigt. Zusätzlich fanden wir eine Rekrutierung atelektatischer Regionen in den dorsalen Lungenabschnitten (s. Abbildung 17, Seite 36). 
Ein inadäquater Atemwegsspitzendruck der durch Beatmung mit hohen Tidalvolumina (> $8 \mathrm{ml} / \mathrm{KG}$ ) und hohem PEEP entstehen kann, schädigt die Lunge sekundär durch Überblähung von bereits geöffneten Lungenregionen, welches zum Einreissen der Alveolarmembran führt. Die von uns in Studie IV eingeführte Methode der Messung von Ventilationsgruppen kann ausschliesslich atelektatische Regionen detektieren, ist jedoch nicht geeignet, um regionale Überblähungen zu detektieren, die eventuell auch dynamisch auftreten können. Aus diesem Grund wurde in Studie V die regionale Atemmechanik untersucht, um einen adäquaten PEEP zur Vermeidung von zyklischem Kollabieren atelektatischer Regionen und obere Grenzwerte des Spitzendruckes zur Vermeidung regionaler Überblähungen zu bestimmen.

In Studie V untersuchten wir regionale Druck-Volumen-Kurven beatmeter Patienten mit akutem Lungenversagen und fanden eine ausgeprägte Heterogenität unterer und oberer Inflektionspunkte. Bisher wurde angenommen, dass der PEEP aus dem unteren Inflektionspunkt einer globalen PV-Kurve bestimmt werden kann (Albaiceta et al. 2004, Crotti et al. 2001, Hickling 1998) und dass der Atemwegsspitzendruck den oberen Inflektionspunkt nicht überschreiten sollte (Matamis et al. 1984). In letzter Zeit wurde jedoch vermutet, dass die Kurvenform einer globalen Druck-Volumen-Kurve durch regionale atemmechanische Eigenschaften der Lunge beeinflusst wird und dass eventuell globale PV-Kurven die Summe verschiedener regionaler PV-Kurven sind. Die Rekrutierung von Atelektasen geschieht deshalb vermutlich entlang der gesamten globalen PVKurve und nicht ausschliesslich am unteren Inflektionspunkt der globalen PVKurve (Albaiceta et al. 2004, Crotti et al. 2001, Hickling 1998, Kunst et al. 2000). 
Dies konnten wir in unseren Ergebnissen bestätigen. Die Medianwerte der regionalen LIP und UIP stimmten mit den LIP und UIP aus den globalen Messungen der Pneumotachographie überein. Jedoch fanden wir eine ausgeprägte Heterogenität der regionalen LIP und UIP sowohl in Hinblick auf die Druckwerte als auch die Anzahl der Regionen und deren örtliche Verteilung (s. Abbildung 18 Seite 39 und Abbildung 19 Seite 40). Hieraus lässt sich vermuten, dass eine Rekrutierung von Atelektasen auch jenseits des unteren Inflektionspunktes der globalen PV-Kurve geschieht. Weiterhin konnten wir nicht bei allen Patienten bei der globalen Druck-Volumen-Kurve eindeutige Inflektionspunkte bestimmen. Dies wurde ebenfalls von anderen Autoren bereits beschrieben (Sydow et al. 1991, Sydow et al. 1993). Bezüglich der ausgeprägten regionalen Heterogenität der LIP und UIP wurden ähnliche Befund bereits im Tierversuch gefunden (Kunst et al. 2000). Wir fanden eine deutliche schwerkraftabhängige Verteilung der LIP und UIP. Ähnliche Ergebnisse wurden ebenfalls bereits in der KunstStudie vorgestellt. Die Rekonstruktion von regionalen Inflektionspunkten hängt jedoch von der örtlichen und zeitlichen Auflösung der verwendeten EIT-Hardware ab (Hahn et al. 2001, Harris et al. 1987). In bisherigen Studien wurde eine örtliche regionale Auflösung von zwei ROI (ventrale und dorsale Thoraxhälfte) innerhalb der untersuchten EIT-Ebene gewählt, wohingegen wir eine Auflösung von 912 ROIs innerhalb der EIT-Ebene untersuchten. Zusätzlich werden Inflektionspunkte durch geringe Samplingfrequenzen überschätzt. Wir verwendeten deshalb eine zeitliche Auflösung von 13 EIT-Bildern pro Sekunde.

Mehrere Autoren verwiesen auf die Bedeutung der regionalen Informationen, die die elektrische Impedanztomographie liefert. Insbesondere 
wurde darauf hingewiesen wie sehr regionale sich von globalen Messwerten unterscheiden (Arnold 2004, Frerichs et al. 2003, van Genderingen et al. 2004, Victorino et al. 2004, Wolf und Arnold 2005). Die Messung der regionalen LIP und UIP bietet den Vorteil sowohl der topographischen als auch histographischen Darstellung. Die histographisch Darstellung zeigt jedoch, dass ein PEEP, der nötig wäre, um in allen Lungenregionen ein zyklischen Kollabieren und Wiedereröffnen zu verhindern, dazu führt, dass in anderen Lungenregionen der UIP überschritten wird und somit ein Atemwegsdruck erreicht wird, der zur Überblähung dieser Regionen führt. Hieraus lässt sich schliessen, dass sowohl Rekruitment als auch Überblähung in Lungen mit akutem Lungenversagen nebeneinander vorliegen. Die Beatmung mit hohen PEEP führt zwangsläufig zu einer gewissen Überblähung (Vieira et al. 1998) bevorzugt in gut belüfteten ventralen Lungenarealen (Gattinoni et al. 1995). Auch dadurch können sekundäre Lungenschäden als Folge eines Barotraumas entstehen. Als Konsequenz muss bei der Beatmungseinstellung ein Kompromiss gefunden werden, zwischen dem Bemühen durch ausreichend hohen PEEP ein zyklisches Kollabieren und Wiedereröffnen geschädigter Lungenareale $\mathrm{zu}$ vermeiden und andererseits gesunde Lungenabschnitte nicht zu stark zu überblähen. Die EIT könnte eventuell hilfreich sein beim Verständnis der Mechanismen der Entstehung des sekundären Lungenversagens. Jedoch wird aus unseren Ergebnissen deutlich, dass der PEEP, der zum Offenhalten aller Lungenregionen beim Lungenversagen nötig ist, aus den globalen PV-Kurven unterschätzt wird. Wir schlossen deshalb aus der Studie V, dass bei Patienten mit akutem Lungenversagen globale PV-Kurven regionale atemmechanische Eigenschaften der Lunge nicht ausreichend beschreiben. 
In der aktuellen Literatur wurde deutlich, dass noch nicht abschliessend geklärt ist, ob eine PV Kurve in Form eines inspiratorischen oder exspiratorischen Manövers durchgeführt werden sollte (Rouby et al. 2002). In einer Studie bei beatmeten Patienten mit frühem akuten Lungenversagen konnte gezeigt werden, dass eine PEEP Einstellung anhand einer exspiratorischen PV-Kurve vorteilhafter für den Patienten ist als anhand einer inspiratorischen PV-Kurve (Albaiceta et al. 2004, Rouby 2004). Prinzipiell ist es möglich mit der EIT exspiratorische PV-Kurven zu konstruieren. Auch die Wahl anderer Manöver zur Konstruktion von PV-Kurven sollte für die EIT unproblematisch sein. In unserer Studie verwendeten wir ein Low-Flow-Manöver, um den Einfluss des Atemwegswiderstandes auf die Atemmechanik zu reduzieren. Diese Methode ist einfach, reproduzierbar und erlaubt die Visualisierung des initialen Anteils einer PV-Kurve (Mankikian et al. 1983). Prinzipiell sollten andere Manöver wie „super syringe“ (Mead et al. 1957), „flow interruption“ (Gottfried et al. 1985), “PEEPWave” (Putensen et al. 1989), "static compliance by automated single steps” (Sydow et al. 1993) und "dynamic loop” (Lu et al. 1999) möglich sein, um PV-Kurven zu generieren. Jedoch benötigen diese Verfahren eventuell eine offline Bearbeitung der PV-Kurven. 
Zusammenfassend lässt sich aus der Studie V schlussfolgern, dass sich mit der EIT regionale LIP und UIP berechnen lassen. Diese Technik ist eventuell hilfreich, um anhand der histographischen Verteilungen möglicherweise den Atemwegsdruck so zu wählen, dass end-exspiratorischer Kollaps und endinspiratorische Überblähung bei Patienten mit Lungenversagen in der Mehrzahl der Regionen vermieden wird.

\section{Zusammenfassung}

Die Überprüfung der EIT mit klinisch etablierten Verfahren der Luftgehalts- und Ventilationsmessung fehlte bis heute. Ein positiver Vergleich der nicht invasiven EIT mit Referenzverfahren bei beatmeten Patienten ist hilfreich, um die regionale Lungenfunktion bettseitig, kontinuierlich überwachen zu können und die Einstellung von Beatmungsgeräten zu optimieren, um regionale Atelektasen und Lungenüberblähung zu verhindern. Kombiniert mit einer Atemwegsdruckmessung kann die EIT bettseitig regionale Atememchanik messen. Das Ziel dieser Habilitationsarbeit war deshalb die Überprüfung der EIT mit klinisch etablierten Verfahren der globalen und regionalen Lungenvolumenund Ventilationsmessung (CT, Ventialtionsszintigraphie und offener Stickstoffauswasch). Desweiteren wurde ein Verfahren entwickelt, dass die bettseitige Überwachung des Einflusses von PEEP auf die regionale Ventilation ermöglicht. Zusätzlich wurde ein Verfahren ermittelt, dass die Messung der regionalen Atemmechanik durch regionale PV-Kurven ermöglicht. Wir fanden eine ausreichend Übereinstimmung der elektrischen Impedanztomographie mit klinischen Methoden der globalen und regionalen Luftgehaltsmessung. Wir 
führten basierend auf diesen Ergebnissen Parameter ein, die bettseitig, kontinuierlich und nicht invasiv die regionale Ventilation und deren Atemmechanik überwachen. Dank der verbesserten EIT-Geräte und verbesserter Datenauswertungsmöglichkeiten wird eine deutliche Zunahme der Verwendung der EIT für zurzeit zumeist wissenschaftliche Fragestellungen beobachtet (Hedenstierna 2004). Zum Einsatz der EIT in der klinischen Routine ist die Entwicklung standardisierter Auswertealgorithmen (Calzia et al. 2005, Demidenko et al. 2005) und Fehlerdiagnosen nötig (Asfaw und Adler 2005). Diese Habilitationsarbeit unterstützt den zukünftigen Einsatz der EIT bei mechanisch beatmeten Patienten zur kontinuierlichen und bettseitigen Überwachung der regionalen Lungenfunktion. 


\section{Danksagung}

Ich möchte mich bei allen Mitarbeitern, Kollegen und Freunden bedanken, die mich bei der Durchführung dieser Habilitationsarbeit unterstützt haben. Ohne die Unterstützung dieser Personen wäre diese Arbeit nicht entstanden. Mein besonderer Dank gilt:

Prof. Dr. med. Gerhard Hellige, Dr. Günter Hahn, PD Dr. Inez Frerichs und Dr. Taras Dudykevych für die Möglichkeit in den Laboren der anästhesiologischen Forschung die faszinierende Technik der elektrischen Impedanztomographie kennen zulernen. Besonders dankbar bin ich dafür, dass sie mir in vielen Gesprächen die elektrische Impedanztomographie näher brachten und mich bei meinen experimentellen und klinischen Studien unterstützten.

Prof. Hilmar Burchardi, der mich für die klinischen als auch wissenschaftlichen Aspekte der Beatmungstherapie des akuten Lungenversagens begeistert hat. In seiner Arbeitsgruppe habe ich gelernt, was Atemmechanik bedeutet. Seiner Unterstützung verdanke ich die Möglichkeit bei Göran Hedenstierna in Schweden wissenschaftlich gearbeitet zu haben.

Prof. Göran Hedenstierna, der mich in Schweden in vielen Gesprächen angeregt und motiviert hat, den „Dingen auf den Grund zu gehen“. Ich freue mich darauf, auch weiterhin mit ihm zusammenarbeiten zu dürfen. 


\section{Literatur}

Adler A, Amyot R, Guardo R, Bates JH, Berthiaume Y (1997): Monitoring changes in lung air and liquid volumes with electrical impedance tomography. $\mathrm{J}$ Appl Physiol 83(5), 1762-1767

Adler A, Shinozuka N, Berthiaume Y, Guardo R, Bates JH (1998): Electrical impedance tomography can monitor dynamic hyperinflation in dogs. J Appl Physiol 84(2), 726-732

Albaiceta GM, Taboada F, Parra D, Luyando LH, Calvo J, Menendez R, Otero J (2004): Tomographic study of the inflection points of the pressure-volume curve in acute lung injury. Am J Respir Crit Care Med 170(10), 1066-1072

Amato MB, Barbas CS, Medeiros DM, Magaldi RB, Schettino GP, Lorenzi-Filho G, Kairalla RA, Deheinzelin D, Munoz C, Oliveira R, Takagaki TY, Carvalho CR (1998): Effect of a protective-ventilation strategy on mortality in the acute respiratory distress syndrome. N Engl J Med 338(6), 347-354

Andrews P, Azoulay E, Antonelli M, Brochard L, Brun-Buisson C, Dobb G, Fagon JY, Gerlach H, Groeneveld J, Mancebo J, Metnitz P, Nava S, Pugin J, Pinsky M, Radermacher P, Richard C, Tasker R, Vallet B (2005): Year in review in intensive care medicine, 2004. I. Respiratory failure, infection, and sepsis. Intensive Care Med 31(1), 28-40 
Andrews PJ, Piper IR, Dearden NM, Miller JD (1990): Secondary insults during intrahospital transport of head-injured patients. Lancet 335(8685), 327-330

Arnold JH (2004): Electrical impedance tomography: on the path to the Holy Grail. Crit Care Med 32(3), 894-895

Asfaw Y, Adler A (2005): Automatic detection of detached and erroneous electrodes in electrical impedance tomography. Physiol Meas 26(2), S175-S183

Barber DC (1990): Quantification in impedance imaging. Clin Phys Physiol Meas 11 Suppl A, 45-56

Barber DC, Brown BH (1984): Applied potential tomography. J Phys E Sci Instrum 17(17), 723-733

Barber DC, Brown BH: Recent developments in applied potential tomographyAPT. In Proceedings of the 9th Conference of Information Processing in Medical Imaging. Ed. S.L. Bacharach, Martinus Nijhoff, Dordrecht 1986. 1986; 106-121

Becker CR, Schätzl M, Schöpg UJ, Brüning R, Reiser MF (1998): Technical bases and aquisition of electron-beam computed tomography. Radiologe 38(38), 987-992 
Bendixen HH, Hedley-Whyte J, Laver MB (1963): Impaired oxygenation in surgical patients during general anesthesia with controlled ventilation: A concept of atelectasis. N Engl J Med 269(269), 991-996

Bercault N, Wolf M, Runge I, Fleury JC, Boulain T (2005): Intrahospital transport of critically ill ventilated patients: a risk factor for ventilator-associated pneumonia--a matched cohort study. Crit Care Med 33(11), 2471-2478

Bernard GR, Artigas A, Brigham KL, Carlet J, Falke K, Hudson L, Lamy M, LeGall JR, Morris A, Spragg R (1994): Report of the American-European Consensus conference on acute respiratory distress syndrome: definitions, mechanisms, relevant outcomes, and clinical trial coordination. Consensus Committee. J Crit Care 9(1), 72-81

Bland JM, Altman DG (1986): Statistical methods for assessing agreement between two methods of clinical measurement. Lancet 1(8476), 307-310

Brismar B, Hedenstierna G, Lundquist H, Strandberg A, Svensson L, Tokics L (1985): Pulmonary densities during anesthesia with muscular relaxation- a proposal of atelectasis. Anesthesiology 62(62), 422-428

Brochard L (2000): Non-invasive ventilation for acute exacerbations of COPD: a new standard of care. Thorax 55(10), 817-818 
Brown BH, Barber DC (1987): Electrical impedance tomography; the construction and application to physiological measurement of electrical impedance images. Med Prog Technol 13(2), 69-75

Burch WM, Sullivan PJ, Lomas FE, Evans VA, McLaren CJ, Arnot RN (1986): Lung ventilation studies with technetium-99m Pseudogas. J Nucl Med 27(6), 842846

Calzia E, Hahn G, Hellige G (2005): Electrical impedance tomography: looking behind the secrets of regional lung function. Intensive care Med 31(11), 14741475

Chiumello D, Pristine G, Slutsky AS (1999): Mechanical ventilation affects local and systemic cytokines in an animal model of acute respiratory distress syndrome. Am J Respir Crit Care Med 160(1), 109-116

Crotti S, Mascheroni D, Caironi P, Pelosi P, Ronzoni G, Mondino M, Marini JJ, Gattinoni L (2001): Recruitment and derecruitment during acute respiratory failure: a clinical study. Am J Respir Crit Care Med 164(1), 131-140

Dambrosio M, Roupie E, Mollet JJ, Anglade MC, Vasile N, Lemaire F, Brochard L (1997): Effects of positive end-expiratory pressure and different tidal volumes on alveolar recruitment and hyperinflation. Anesthesiology 87(3), 495-503 
Darling RC, Richards DW, Cournant A (1940): Studies on intrapulmonary mixture of gases. Open curcuit method for measuring residual air. J Clin Invest 19(19), 609-618

Dellinger RP, Carlet JM, Masur H, Gerlach H, Calandra T, Cohen J, GeaBanacloche J, Keh D, Marshall JC, Parker MM, Ramsay G, Zimmerman JL, Vincent JL, Levy MM (2004): Surviving Sepsis Campaign guidelines for management of severe sepsis and septic shock. Crit Care Med 32(3), 858-873

Demidenko E, Hartov A, Soni N, Paulsen KD (2005): On optimal current patterns for electrical impedance tomography. IEEE Trans Biomed Eng 52(2), 238-248

Downey GP, Granton JT (1997): Mechanisms of acute lung injury. Curr Opin Pulm Med 3(3), 234-241

Dreyfuss D, Saumon G (1998): Ventilator-induced lung injury: lessons from experimental studies. Am J Respir Crit Care Med 157(1), 294-323

Drummond GB (1998): Computed tomography and pulmonary measurements. $\mathrm{Br}$ J Anaesth 80(5), 665-671

Faes TJ, van der Meij HA, de Munck JC, Heethaar RM (1999): The electric resistivity of human tissues (100 Hz-10 MHz): a meta- analysis of review studies. Physiol Meas 20(4), R1-10 
Flatten H, Aardal S, Hevroy E (1998): Improved oxygenation using the prone positioning in patients with ARDS. Acta Anaesthesiol Scandinavica 42(42), 329334

Fleisch A (1925): Der Pneumotachograph: ein Apparat zur

Geschwindigkeitsregulierung der Atemluft. Plügers Arch ges Physio 209(209), $713-722$

Frerichs I (2000): Electrical impedance tomography (EIT) in applications related to lung and ventilation: a review of experimental and clinical activities. Physiol Meas 21(2), R1-21

Frerichs I, Dargaville PA, Dudykevych T, Rimensberger PC (2003): Electrical impedance tomography: a method for monitoring regional lung aeration and tidal volume distribution? Intensive Care Med 29(12), 2312-2316

Frerichs I, Hinz J, Herrmann P, Weisser G, Hahn G, Dudykevych T, Quintel M, Hellige G (2002a): Detection of local lung air content by electrical impedance tomography compared with electron beam CT. J Appl Physiol 93(2), 660-666

Frerichs I, Hinz J, Herrmann P, Weisser G, Hahn G, Quintel M, Hellige G (2002b): Regional lung perfusion as determined by electrical impedance tomography in comparison with electron beam CT imaging. IEEE Trans Med Imaging 21(6), 646-652 
Gattinoni L, D'Andrea L, Pelosi P, Vitale G, Pesenti A, Fumagalli R (1993):

Regional effects and mechanism of positive end-expiratory pressure in early adult respiratory distress syndrome. JAMA 269(16), 2122-2127

Gattinoni L, Pelosi P, Crotti S, Valenza F (1995): Effects of positive endexpiratory pressure on regional distribution of tidal volume and recruitment in adult respiratory distress syndrome. Am J Respir Crit Care Med 151(6), 18071814

Gattinoni L, Pesenti A, Avalli L, Rossi F, Bombino M (1987): Pressure-volume curve of total respiratory system in acute respiratory failure. Computed tomographic scan study. Am Rev Respir Dis 136(3), 730-736

Gattinoni L, Pesenti A, Bombino M, Baglioni S, Rivolta M, Rossi F, Rossi G, Fumagalli R, Marcolin R, Mascheroni D, . (1988): Relationships between lung computed tomographic density, gas exchange, and PEEP in acute respiratory failure. Anesthesiology 69(6), 824-832

Gattinoni L, Pesenti A, Caspani ML, Pelizzola A, Mascheroni D, Marcolin R, Iapichino G, Langer M, Agostoni A, Kolobow T, . (1984): The role of total static lung compliance in the management of severe ARDS unresponsive to conventional treatment. Intensive care Med 10(3), 121-126 
Gattinoni L, Presenti A, Torresin A, Baglioni S, Rivolta M, Rossi F, Scarani F, Marcolin R, Cappelletti G (1986): Adult respiratory distress syndrome profiles by computed tomography. J Thorac Imaging 3(1), 25-30

Gottfried SB, Rossi A, Higgs BD, Calverley PM, Zocchi L, Bozic C, Milic-Emili J (1985): Noninvasive determination of respiratory system mechanics during mechanical ventilation for acute respiratory failure. Am Rev Respir Dis 131(3), $414-420$

Gunnarsson L, Tokics L, Gustavsson H, Hedenstierna G (1991): Influence of age on atelectasis formation and gas exchange impairment during general anaesthesia. Br J Anaesth 66(4), 423-432

Hahn G, Beer M, Frerichs I, Dudykevych T, Schroder T, Hellige G (2000): A simple method to check the dynamic performance of electrical impedance tomography systems. Physiol Meas 21(1), 53-60

Hahn G, Frerichs I, Golisch W, Kurpitz M, Schroder T, Hellige G (1997):

Determining local lung ventilation by functional electrical impedance tomography under clinical circumstances. Biomed Tech (Berl) 42 Suppl(42), 213-214

Hahn G, Frerichs I, Kleyer M, Hellige G (1996): Local mechanics of the lung tissue determined by functional EIT. Physiol Meas 17 Suppl 4A, A159-A166 
Hahn G, Hartung C, Hellige G (1998): 6.2.5 Bestimmung der Grösse minimal erfassbarer Areale mit Ventilationsstörungen aus: Elektrische Impedanztomographie (EIT) als Methode zur regionalen Beurteilung der Lungenventilation; Gustav Fischer Verlag, Mainz 77-77

Hahn G, Sipinkova I, Baisch F, Hellige G (1995): Changes in the thoracic impedance distribution under different ventilatory conditions. Physiol Meas 16(3 Suppl A), A161-A173

Hahn G, Thiel F, Dudykevych T, Frerichs I, Gersing E, Schroder T, Hartung C, Hellige G (2001): Quantitative evaluation of the performance of different electrical tomography devices. Biomed Tech (Berl) 4(46), 91-95

Harris ND, Suggett AJ, Barber DC, Brown BH (1987): Applications of applied potential tomography (APT) in respiratory medicine. Clin Phys Physiol Meas 8 Suppl A(8), 155-165

Hedenstierna G (1993): The recording of FRC - Is it of importance and can it be made simple? Intensive care Med 19(7), 365-366

Hedenstierna G (2004): Using electric impedance tomography to assess regional ventilation at the bedside. Am J Respir Crit Care Med 169(7), 777-778

Hedenstierna G, Strandberg A, Brismar B, Lundquist H, Svensson L, Tokics L (1985): Functional residual capacity, thoracoabdominal dimensions, and central 
blood volume during general anesthesia with muscle paralysis and mechanical ventilation. Anesthesiology 62(3), 247-254

Hedenstierna G, Tokics L, Strandberg A, Lundquist H, Brismar B (1986):

Correlation of gas exchange impairment to development of atelectasis during anaesthesia and muscle paralysis. Acta Anaesthesiol Scand(30), 183-191

Hickling KG (1998): The pressure-volume curve is greatly modified by recruitment. A mathematical model of ARDS lungs. Am J Respir Crit Care Med 158(1), 194-202

Hinz J, Hahn G, Neumann P, Sydow M, Mohrenweiser P, Hellige G, Burchardi H (2003a): End-expiratory lung impedance change enables bedside monitoring of end- expiratory lung volume change. Intensive Care Med 29(1), 37-43

Hinz J, Moerer O, Neumann P, Dudykevych T, Frerichs I, Hellige G, Quintel M (2006): Regional pulmonary pressure volume curves in mechanically ventilated patients with acute respiratory failure measured by electrical impedance tomography. Acta Anaesthesiol Scand 50(3), 331-339

Hinz J, Moerer O, Neumann P, Dudykevych T, Hellige G, Quintel M (2005): Effect of positive end-expiratory-pressure on regional ventilation in patients with acute lung injury evaluated by electrical impedance tomography. Eur J Anaesthesiol 22(11), 817-825 
Hinz J, Neumann P, Dudykevych T, Anderson LG, Wrigge H, Burchardi H, Hedenstierna G (2003b): Regional ventilation by Electrical Impedance Tomography-A comparison with ventilation scintigraphy in pigs. Chest(124), $314-322$

Hudson LD, Steinberg KP (1999): Epidemiology of acute lung injury and ARDS. Chest 116(1 Suppl), 74S-82S

Kacmarek RM (1999): Ventilator-associated lung injury. Int Anesthesiol Clin 37(3), 47-64

Kunst PW, Bohm SH, de Vazquez A, Amato MB, Lachmann B, Postmus PE, de Vries PM (2000): Regional pressure volume curves by electrical impedance tomography in a model of acute lung injury. Crit Care Med 28(1), 178-183

Kunst PW, Vonk NA, Hoekstra OS, Postmus PE, de Vries PM (1998): Ventilation and perfusion imaging by electrical impedance tomography: a comparison with radionuclide scanning. Physiol Meas 19(4), 481-490

Lachmann B (1992): Open up the lung and keep the lung open. Intensive care Med 18(6), 319-321

Lim CM, Soon LS, Seoung LJ, Koh Y, Sun ST, Do LS, Sung KW, Kim DS, Dong KW (2003): Morphometric effects of the recruitment maneuver on saline-lavaged canine lungs. A computed tomographic analysis. Anesthesiology 99(1), 71-80 
Lu Q, Vieira SR, Richecoeur J, Puybasset L, Kalfon P, Coriat P, Rouby JJ (1999):

A simple automated method for measuring pressure-volume curves during mechanical ventilation. Am J Respir Crit Care Med 159(1), 275-282

Mankikian B, Lemaire F, Benito S, Brun-Buisson C, Harf A, Maillot JP, Becker J (1983): A new device for measurement of pulmonary pressure-volume curves in patients on mechanical ventilation. Crit Care Med 11(11), 897-901

Matamis D, Lemaire F, Harf A, Brun-Buisson C, Ansquer JC, Atlan G (1984): Total respiratory pressure-volume curves in the adult respiratory distress syndrome. Chest 86(1), 58-66

Mead J, Whittenberger JL, Radford EP (1957): Surface tension as a factor in pulmonary volume-pressure hysteresis. J Appl Physiol 2(10), 191-196

Metherall P, Barber DC, Smallwood RH, Brown BH (1996): Three-dimensional electrical impedance tomography. Nature 380(6574), 509-512

Murray JF, Matthay MA, Luce JM, Flick MR (1988): An expanded definition of the adult respiratory distress syndrome. Am Rev Respir Dis 138(3), 720-723

Neumann P, Berglund JE, Mondejar EF, Magnusson A, Hedenstierna G (1998a): Dynamics of lung collapse and recruitment during prolonged breathing in porcine lung injury. J Appl Physiol 85(4), 1533-1543 
Neumann P, Berglund JE, Mondejar EF, Magnusson A, Hedenstierna G (1998b):

Effect of different pressure levels on the dynamics of lung collapse and recruitment in oleic-acid-induced lung injury. Am J Respir Crit Care Med 158, $1636-1643$

Neumann P, Hedenstierna G (2001): Ventilation-perfusion distributions in different porcine lung injury models. Acta Anaesthesiol Scand 45(1), 78-86

Nopp P, Rapp E, Pfutzner H, Nakesch H, Ruhsam C (1993): Dielectric properties of lung tissue as a function of air content. Phys Med Biol 38(6), 699-716

Pelosi P, Gattinoni L (1996): Mechanical ventilation in adult respiratory distress syndrome: a good friend or a secret killer? Current Opinion Anaesthesiol 9(9), $515-522$

Pinhu L, Whitehead T, Evans T, Griffiths M (2003): Ventilator-associated lung injury. Lancet 361(9354), 332-340

Plotz FB, Slutsky AS, van Vught AJ, Heijnen CJ (2004): Ventilator-induced lung injury and multiple system organ failure: a critical review of facts and hypotheses. Intensive Care Med 30(10), 1865-1872

Plotz FB, Vreugdenhil HA, van Vught AJ, Heijnen CJ (2003): Mechanical ventilation and multiple organ failure. Lancet 361(9369), 1654-1654 
Putensen C, Baum M, Putz G (1989): PEEP-Welle: Ein automatisiertes Verfahren zur bettseitigen Bestimmung der Volumen/Druck-Beziehung der Lunge beatmeter Patienten. Anaesthesist 38(4), 214-219

Putensen C, Zech S, Wrigge H, Zinserling J, Stuber F, von Spiegel T, Mutz N (2001): Long-term effects of spontaneous breathing during ventilatory support in patients with acute lung injury. Am J Respir Crit Care Med 164(1), 43-49

Puybasset L, Gusman P, Muller JC, Cluzel P, Coriat P, Rouby JJ (2000): Regional distribution of gas and tissue in acute respiratory distress syndrome. III. Consequences for the effects of positive end-expiratory pressure. CT Scan ARDS Study Group. Adult Respiratory Distress Syndrome. Intensive Care Med 26(9), $1215-1227$

Rabbani KS, Kabir AM (1991): Studies on the effect of the third dimension on a two-dimensional electrical impedance tomography system. Clin Phys Physiol Meas 12(4), 393-402

Ranieri VM, Suter PM, Tortorella C, De Tullio R, Dayer JM, Brienza A, Bruno F, Slutsky AS (1999): Effect of mechanical ventilation on inflammatory mediators in patients with acute respiratory distress syndrome: a randomized controlled trial. JAMA 282(1), 54-61 
Reber A, Engberg G, Wegenius G, Hedenstierna G (1996): Lung aeration. The effect of pre-oxygenation and hyperoxygenation during total intravenous anaesthesia. Anaesthesia 51(8), 733-737

Rothen HU, Neumann P, Berglund JE, Valtysson J, Magnusson A, Hedenstierna G (1999): Dynamics of re-expansion of atelectasis during general anaesthesia. $\mathrm{Br}$ J Anaesth 82(4), 551-556

Rothen HU, Sporre B, Engberg G, Wegenius G, Hogman M, Hedenstierna G (1995): Influence of gas composition on recurrence of atelectasis after a reexpansion maneuver during general anesthesia. Anesthesiology 82(4), 832-842 Rouby JJ (2004): Optimizing lung aeration in positive end-expiratory pressure. Am J Respir Crit Care Med 170(10), 1039-1040

Rouby JJ, Lu Q, Goldstein I (2002): Selecting the right level of positive endexpiratory pressure in patients with acute respiratory distress syndrome. Am J Respir Crit Care Med 165(8), 1182-1186

Schuster DP (1994): ARDS: clinical lessons from the oleic acid model of acute lung injury. Am J Respir Crit Care Med 149(1), 245-260

Slutsky AS, Tremblay LN (1998): Multiple system organ failure. Is mechanical ventilation a contributing factor? Am J Respir Crit Care Med 157(6 Pt 1), 17211725 
Smith I, Fleming S, Cernaianu A (1990): Mishaps during transport from the intensive care unit. Crit Care Med 18(3), 278-281

Sydow M, Burchardi H, Ephraim E, Zielmann S, Crozier TA (1994): Long-term effects of two different ventilatory modes on oxygenation in acute lung injury. Comparison of airway pressure release ventilation and volume-controlled inverse ratio ventilation. Am J Respir Crit Care Med 149(6), 1550-1556

Sydow M, Burchardi H, Zinserling J, Crozier TA, Denecke T, Zielmann S (1993): Intrinsic PEEP determined by static pressure-volume curves--application of a novel automated occlusion method. Intensive care Med 19(3), 166-171

Sydow M, Burchardi H, Zinserling J, Ische H, Crozier TA, Weyland W (1991): Improved determination of static compliance by automated single volume steps in ventilated patients. Intensive care Med 17(2), 108-114

Sykes MK (1991): Does mechanical ventilation damage the lung? Acta Anaesthesiol Scand Suppl 95(95), 35-38

Sykes MK, Young WE, Robinson BE (1965): Oxygenation during anaesthesia with controlled ventilation. Br J Anaesth 37, 314-325

The Acute Respiratory Distress Syndrome Network (2000): Ventilation with lower tidal volumes as compared with traditional tidal volumes for acute lung 
injury and the acute respiratory distress syndrome. N Engl J Med 342(342), 13011308

van Beek EJ, Wild JM, Kauczor HU, Schreiber W, Mugler JP, III, de Lange EE (2004): Functional MRI of the lung using hyperpolarized 3-helium gas. J Magn Reson Imaging 20(4), 540-554

van Genderingen HR, van Vught AJ, Jansen JR (2004): Regional lung volume during high-frequency oscillatory ventilation by electrical impedance tomography. Crit Care Med 32(3), 787-794

Venegas JG, Harris RS, Simon BA (1998): A comprehensive equation for the pulmonary pressure-volume curve. J Appl Physiol 84(1), 389-395

Victorino JA, Borges JB, Okamoto VN, Matos GF, Tucci MR, Caramez MP, Tanaka H, Sipmann FS, Santos DC, Barbas CS, Carvalho CR, Amato MB (2004): Imbalances in regional lung ventilation: a validation study on electrical impedance tomography. Am J Respir Crit Care Med 169(7), 791-800

Vieira SR, Puybasset L, Richecoeur J, Lu Q, Cluzel P, Gusman PB, Coriat P, Rouby JJ (1998): A lung computed tomographic assessment of positive endexpiratory pressure-induced lung overdistension. Am J Respir Crit Care Med 158(5 Pt 1), 1571-1577 
Vonk NA, Kunst PW, Janse A, Smulders RA, Heethaar RM, Postmus PE, Faes TJ, de Vries PM (1997): Validity and reproducibility of electrical impedance tomography for measurement of calf blood flow in healthy subjects. Med Biol Eng Comput 35(2), 107-112

Wagner PD, Saltzman HA, West JB (1974): Measurement of continuous distributions of ventilation-perfusion ratios: theory. J Appl Physiol 36(5), 588-599

Warren J, Fromm RE, Jr., Orr RA, Rotello LC, Horst HM (2004): Guidelines for the inter- and intrahospital transport of critically ill patients. Crit Care Med 32(1), 256-262

Wolf GK, Arnold JH (2005): Noninvasive assessment of lung volume: respiratory inductance plethysmography and electrical impedance tomography. Crit Care Med 33(3 Suppl), S163-S169

Wrigge H, Golisch W, Zinserling J, Sydow M, Almeling G, Burchardi H (1999):

Proportional assist versus pressure support ventilation: effects on breathing pattern and respiratory work of patients with chronic obstructive pulmonary disease. Intensive care Med 25(8), 790-798

Wrigge H, Sydow M, Zinserling J, Neumann P, Hinz J, Burchardi H (1998): Determination of functional residual capacity (FRC) by multibreath nitrogen washout in a lung model and in mechanically ventilated patients. Accuracy 
depends on continuous dynamic compensation for changes of gas sampling delay time. Intensive Care Med 24(5), 487-493

Wrigge H, Zinserling J, Neumann P, Defosse J, Magnusson A, Putensen C, Hedenstierna G (2003): Spontaneous breathing improves lung aeration in oleic acid-induced lung injury. Anesthesiology 99(2), 376-384

Wtorek J, Polinski A (2005): The contribution of blood-flow-induced conductivity changes to measured impedance. IEEE Trans Biomed Eng 52(1), 41-49 


\title{
Detection of local lung air content by electrical impedance tomography compared with electron beam CT
}

\author{
INÉZ FRERICHS, ${ }^{1}$ JOSÉ HINZ, ${ }^{1}$ PETER HERRMANN, ${ }^{2}$ GERALD WEISSER,${ }^{3}$ GÜNTER HAHN,${ }^{1}$ \\ TARAS DUDYKEVYCH, ${ }^{1}$ MICHAEL QUINTEL,${ }^{2}$ AND GERHARD HELLIGE ${ }^{1}$ \\ ${ }^{1}$ Department of Anesthesiological Research, Center of Anesthesiology, Emergency \\ and Intensive Care Medicine, University of Göttingen, D-37075 Göttingen; \\ and Institutes of ${ }^{2}$ Anesthesiology and Operative Intensive Care and ${ }^{3}$ Clinical \\ Radiology, University Hospital Mannheim, D-68167 Mannheim, Germany
}

Received 31 January 2002; accepted in final form 13 April 2002

\begin{abstract}
Frerichs, Inéz, José Hinz, Peter Herrmann, Gerald Weisser, Günter Hahn, Taras Dudykevych, Michael Quintel, and Gerhard Hellige. Detection of local lung air content by electrical impedance tomography compared with electron beam CT. J Appl Physiol 93: 660-666, 2002. First published April 15, 2002; 10.1152/japplphysiol.00081.2002.— The aim of the study was to validate the ability of electrical impedance tomography (EIT) to detect local changes in air content, resulting from modified ventilator settings, by comparing EIT findings with electron beam computed tomography (EBCT) scans obtained under identical steady-state conditions. The experiments were carried out on six anesthetized supine pigs ventilated with five tidal volumes $(\mathrm{VT})$ at three positive end-expiratory pressure (PEEP) levels. The lung air content changes were determined both by EIT (Goe-MF1 system) and EBCT (Imatron C-150XP scanner) in six regions of interest, located in the ventral, middle, and dorsal areas of each lung, with respect to the reference air content at the lowest $\mathrm{VT}_{\mathrm{T}}$ and PEEP, as a change in either local electrical impedance or lung tissue density. An increase in local air content with $\mathrm{VT}_{\mathrm{T}}$ and PEEP was identified by both methods at all regions studied. A good correlation between the changes in lung air content determined by EIT and EBCT was revealed. Mean correlation coefficients in the ventral, middle, and dorsal regions were 0.81 , 0.87 , and 0.93 , respectively. The study confirms that EIT is a suitable, noninvasive method for detecting regional changes in air content and monitoring local effects of artificial ventilation.

noninvasive monitoring; electron beam computed tomography; positive end-expiratory pressure; ventilation distribution
\end{abstract}

THE TOPOGRAPHICAL DISTRIBUTION of inspired air in the lungs in mechanically ventilated patients is influenced by many factors, e.g., the type of ventilatory support, the therapeutic procedures performed, underlying lung pathology, and body position. To assess and optimize the effect of the induced therapy, including artificial ventilation, on lung function, for instance with the aim to reduce the occurrence of atelectasis and shunt, it is necessary that the information on regional lung venti-

Address for reprint requests and other correspondence: I. Frerichs, Dept. of Anesthesiological Research, Center of Anesthesiology, Emergency and Intensive Care Medicine, TL 195, Univ. of Göttingen, Robert-Koch-Str. 40, D-37075 Göttingen, Germany (E-mail: isipink@gwdg.de). lation be continuously available at the bedside. However, this is not possible at present. The feedback information on the functional state of lungs is incomplete, as it is obtained by diagnostic methods, providing only global and not regional parameters of lung function (e.g., blood-gas analysis), or by imaging techniques (e.g., chest radiography and computed tomography), making morphological lung data available at rather long time intervals. In the latter case, the examinations of patients can often not be performed at the bedside, and the patients are exposed to increased radiation.

The noninvasive, radiation-free imaging technique of electrical impedance tomography (EIT) has recently been proposed as a new tool for monitoring regional lung ventilation in clinical settings $(4,7)$. The principle of EIT is based on the measurement of electrical voltages at the surface of the chest, resulting from repeated applications of small electrical currents to the body. The collected EIT data are transformed into two-dimensional images of the distribution of electrical impedance in the chest. The electrical properties of the lung tissue differ significantly from those of other thoracic tissues (9) and, moreover, vary quasi-periodically with ventilation. For this reason, EIT scanning is particularly suitable for assessing the lung function. The EIT scans can be acquired with a good time resolution of a few tens of scans per second and further processed to provide quantitative parameters characterizing several aspects of the local lung function $(5,11,16,25)$.

In view of the probable future use of EIT in monitoring the local lung function in artificially ventilated patients, the aim of our study was to check the ability of this technique to detect local changes in pulmonary air content, resulting from an adjustment of tidal volume (VT) and positive end-expiratory pressure (PEEP), by using an advanced EIT technology. The EIT results were validated by a comparison with the electron beam computed tomography (EBCT) findings obtained under the same steady-state conditions.

The costs of publication of this article were defrayed in part by the payment of page charges. The article must therefore be hereby marked "advertisement" in accordance with 18 U.S.C. Section 1734 solely to indicate this fact. 


\section{METHODS}

The experiments were performed on six anesthetized pigs (body weight: 20-22 kg). The study was approved by the university and state committee for animal care and adhered to the guidelines on animal experimentation. The animals were at first sedated by azaperon $(0.25 \mathrm{ml} / \mathrm{kg}$ body wt). Anesthesia was achieved by a continuous intravenous administration of ketamine $(200 \mathrm{mg} / \mathrm{h})$ and propofol $(100 \mathrm{mg} / \mathrm{h})$. Vecuronium bromide $(0.1 \mathrm{mg} / \mathrm{kg}$ body wt) was used for muscle paralysis. The animals were tracheotomized, intubated, and mechanically ventilated in a volume-controlled, continuous positive pressure mode of ventilation (Siemens Servo Ventilator 300, Siemens-Elema, Solna, Sweden) at a rate of 10 breaths/min with an inspiration-to-expiration ratio of 1:1.3. Initial ventilator settings were as follows: VT, $240 \mathrm{ml}$; $\mathrm{PEEP}, 5 \mathrm{cmH}_{2} \mathrm{O}$, and fractional concentration of oxygen in inspired gas, 0.40. In the later course of each experimental session, the animals were ventilated at 15 distinct ventilatory patterns that were achieved by a combination of five VT $(200,300,400,500,600 \mathrm{ml})$ with three PEEP values $(2,7,12$ $\mathrm{cmH}_{2} \mathrm{O}$ ). At each ventilatory pattern chosen, EIT and EBCT scanning were performed immediately, one after another, under steady-state conditions. The animals were studied in a supine posture.

EIT. EIT measurements were performed with the Göttingen EIT system Goe-MF 1 (12). A set of $16 \mathrm{X}$-ray transparent electrocardiogram electrodes (Blue Sensor BR-50-K, Medicotest, Ølstykke, Denmark) was placed on the thoracic circumference approximately at the level of the sixth intercostal space (medioclavicular line). Figure 1 shows the measuring principle of EIT based on the rotating injection of small alternating electrical currents $(50 \mathrm{kHz}, 5 \mathrm{~mA}$ root mean square) through an array of surface electrodes and the measurements of resulting potential differences on the same electrodes. Both the application of excitation currents and the voltage measurements were always carried out between adjacent pairs of electrodes. During one complete scanning cycle, current injections were performed through all 16 drive electrode pairs. Each application of current to the body was followed by the measurement of potential differences from non-current-carrying pairs of electrodes. This means that a total of 208 values of potential differences were collected during each scanning cycle. The EIT image reconstruction (i.e., the generation of two-dimensional scans of the distribution of electrical impedance in the chest cross section defined by the array of attached electrodes) was achieved by a modified version of the filtered back-projection algorithm (1), with

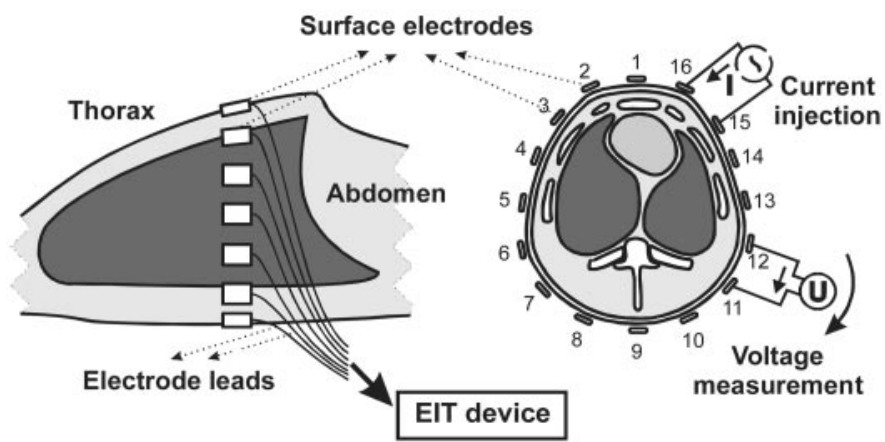

Fig. 1. Measuring principle of electrical impedance tomography (EIT). Electrical excitation currents (I) are consecutively applied between pairs of adjacent surface electrodes (1-16). After each current injection, resulting voltages $(\mathrm{U})$ are measured between the remaining electrode pairs.
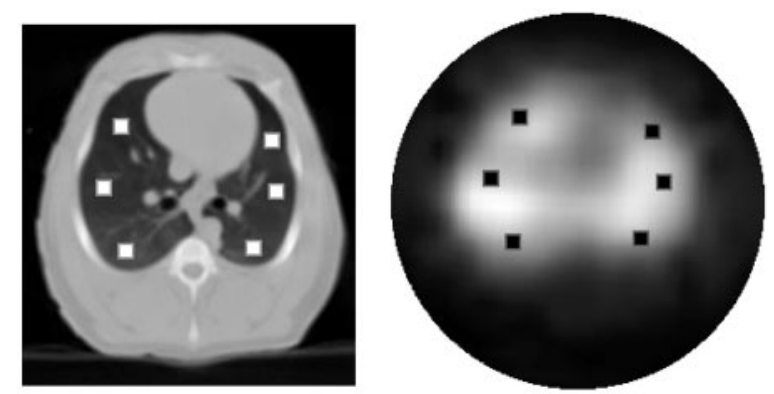

Fig. 2. Anatomic electron beam computed tomography (EBCT) image of chest morphology (left) and functional EIT image of regional lung ventilation (right) with 6 selected regions of interest (ROI).

a resolution of $32 \times 32$ pixels. The output of the image reconstruction procedure per one scanning cycle was 912 values of relative impedance change inscribed in a circular image region. The scanning rate was 13 scans/s, and the scanning period was $77 \mathrm{~s}$. This means that a total of 1,000 EIT scans were collected in one transverse thoracic plane during each scanning period.

$E B C T$. EBCT measurements were carried out with the EBCT scanner C-150XP (Imatron, San Francisco, CA) by using the so-called multislice scanning mode (2). During one scanning period lasting $12 \mathrm{~s}$, a total of 160 scans were collected in four transverse slices (i.e., 40 scans/slice). The scans were generated from the measurement of the X-ray attenuation, depending on the density of the thoracic structures. The values were given in Hounsfield units (HU). The scanning rate was $3.3 \mathrm{scans} / \mathrm{s}$, with an exposure time of 50 ms. The EBCT data were acquired without any movement of the animal in the scanner and spanned $3.8 \mathrm{~cm}$ of the chest. The EBCT scanning was performed in the same part of the chest as EIT scanning. The EBCT image resolution was $256 \times 256$ pixels.

Off-line data analysis. The initial step of the off-line data analysis was the definition of six regions of interest (ROI) in the ventral, middle, and dorsal areas of the right and left lungs (Fig. 2). These ROIs were defined for each individual animal during each ventilatory pattern studied. In the case of EBCT scanning, the ROIs were selected from one of the scans obtained in the last but caudal chest slice during midinspiration (Fig. 2, left). Care was taken that no large pulmonary vessels or bronchi were present in the ROIs at any instant of the respiratory cycle. (For instance, the vessels filled with blood exhibit a much higher tissue density than the air-filled lung tissue, and their occurrence in the ROI would have introduced an error during later data evaluation.) The ROI dimensions were $10 \times 10$ pixels. In the case of EIT scanning, a so-called functional EIT image of regional lung ventilation was generated at first from each set of 1,000 EIT scans by a procedure developed in our laboratory and described in detail in several publications (e.g., Refs. 6, 11). Briefly, this evaluation procedure calculates the local variation of electrical impedance in the thoracic cross section with time and generates images showing the distribution of this parameter in the chest. In these functional EIT images, the lung regions become visible because of large impedance variations caused by the periodic intrapulmonary gas volume changes during ventilation. The functional EIT images of regional lung ventilation were obtained during each ventilatory pattern studied, and the six ROIs were each time defined in the lung regions on the basis of the formerly radiographically located EBCT ROIs (Fig. 2, right). The EIT ROI dimensions were $2 \times$ 
2 pixels. The EIT and EBCT ROIs were of comparable absolute size.

To estimate the average local air content during each ventilatory pattern, the sets of EBCT scans acquired during each individual scanning period were averaged. In this way, the average tissue density in a transverse chest slice of 3.8 $\mathrm{cm}$ was determined. A similar procedure was applied to the EIT data. The original sets of EIT scans collected during all scanning periods were also averaged. The averaged EIT data were representative of the mean electrical properties of the tissues in the same chest slice as during EBCT scanning. In the lung regions, the time-averaged EBCT and EIT data characterized the density and the electrical properties of the pulmonary tissue at an average lung volume during the individual ventilatory settings.

To quantify the changes in regional lung air content associated with the variations of the ventilatory settings, the following approach was used. The changes in local air content were determined in the selected EBCT and EIT ROIs in terms of a change in local average lung density and relative impedance change with respect to a reference air content. The reference lung air content was the average air content at the lowest VT $(200 \mathrm{ml})$ and PEEP $\left(2 \mathrm{cmH}_{2} \mathrm{O}\right)$ studied. The calculated changes in lung density and electrical impedance were plotted as a function of VT at all PEEP levels, showing the effect of the ventilatory pattern on regional lung air content. Furthermore, the correlation between the changes in lung air content determined by EIT and EBCT was determined.

One-way ANOVA was applied to check the effect of $\mathrm{VT}_{\mathrm{T}}$, PEEP, and location of the ROI in the lungs on the calculated changes in lung air content. $P<0.05$ was considered significant.

\section{RESULTS}

A total of 15,500 EBCT scans and 98,000 EIT scans were acquired in six animals during mechanical ventilation at 15 different ventilator settings. The local changes in lung air content occurring as a result of modified ventilatory parameters were quantified both by EIT and EBCT in six ROIs located in the ventral, middle, and dorsal areas of both lungs. The results are shown in Figs. 3-5.

The following changes in regional lung air content, depending on $\mathrm{V}_{\mathrm{T}}$ and PEEP, were consistently identified both by EIT and EBCT. 1) A rise in VT increased the local lung air content at all lung regions and all PEEP levels studied. 2) The highest increase in lung air content with $\mathrm{V}_{\mathrm{T}}$ was observed in the dorsal (dependent) and the smallest in the ventral (nondependent) lung regions. 3) The local differences in the steepness of the lung air content increase with VT were more pronounced at lower PEEP levels of 2 and $7 \mathrm{cmH}_{2} \mathrm{O}$ than at $12 \mathrm{cmH}_{2} \mathrm{O}$. 4) PEEP increased the lung air content at all lung regions studied. 5) The highest increase in lung air content with PEEP was found in the dorsal (dependent) lung regions.

The changes in local lung air content obtained by EBCT and EIT were essentially the same. The correlation between the lung air content changes identified by EBCT and EIT at all $\mathrm{V}_{\mathrm{T}}$ and PEEP values studied was good and is shown in Fig. 6. The best correlation between the EBCT and EIT data was found in the

Fig. 3. Change in local lung air content dependent on tidal volume (VT) at a positive end-expiratory pressure (PEEP) of $2 \mathrm{cmH}_{2} \mathrm{O}$ at 6 ROIs, determined by EBCT (top) and EIT (bottom), with respect to the average lung air content during ventilation at $\mathrm{VT}_{\mathrm{T}}=200 \mathrm{ml}$ and $\mathrm{PEEP}=2 \mathrm{cmH}_{2} \mathrm{O}$. Values are means $\pm \mathrm{SD}$. HU, Hounsfield units; rel, relative; $\Delta$, change; $\mathrm{Z}$, impedance; AU, arbitrary units.

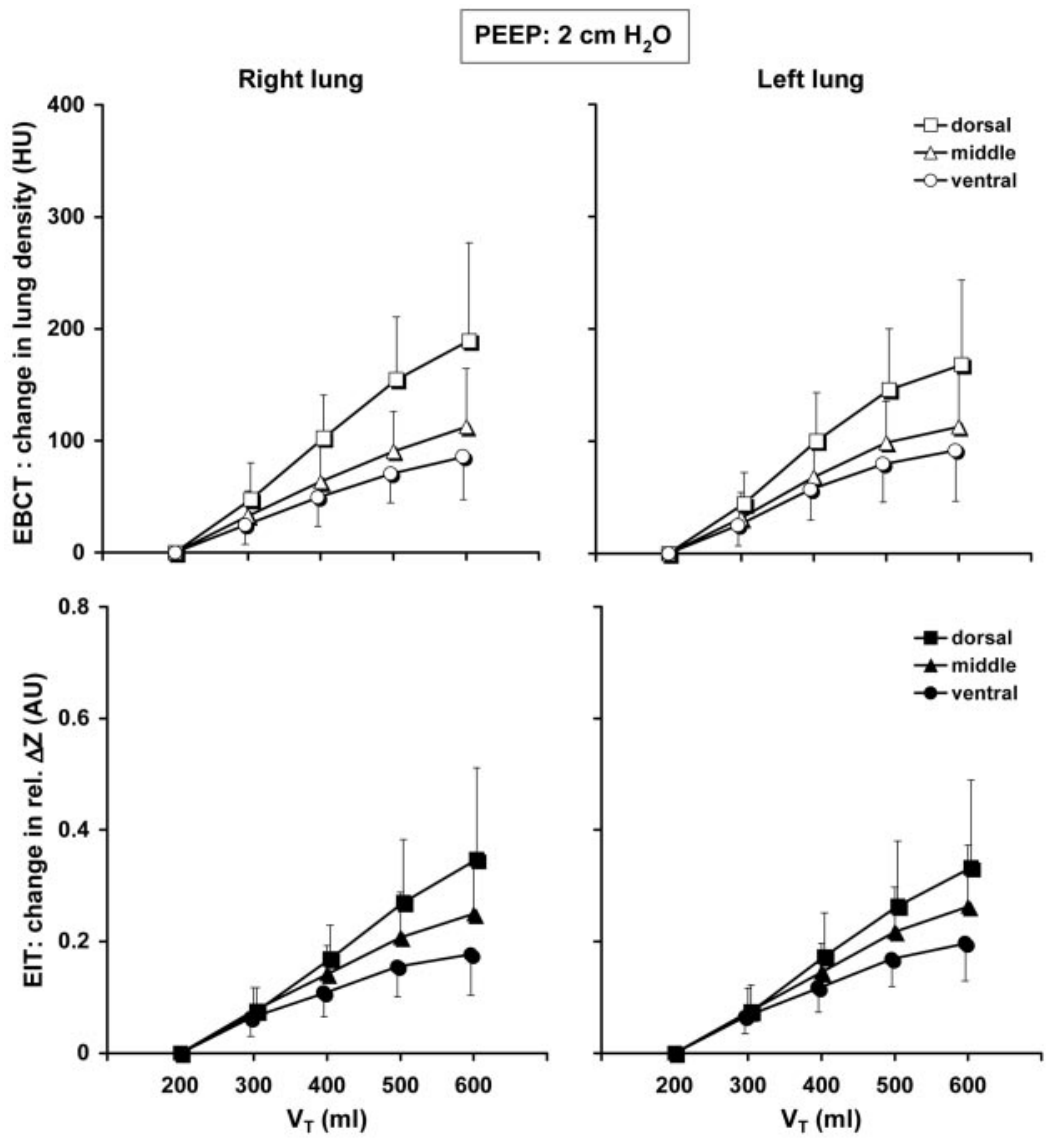

J Appl Physiol • vOL 93 • AUGUST 2002 • www.jap.org 

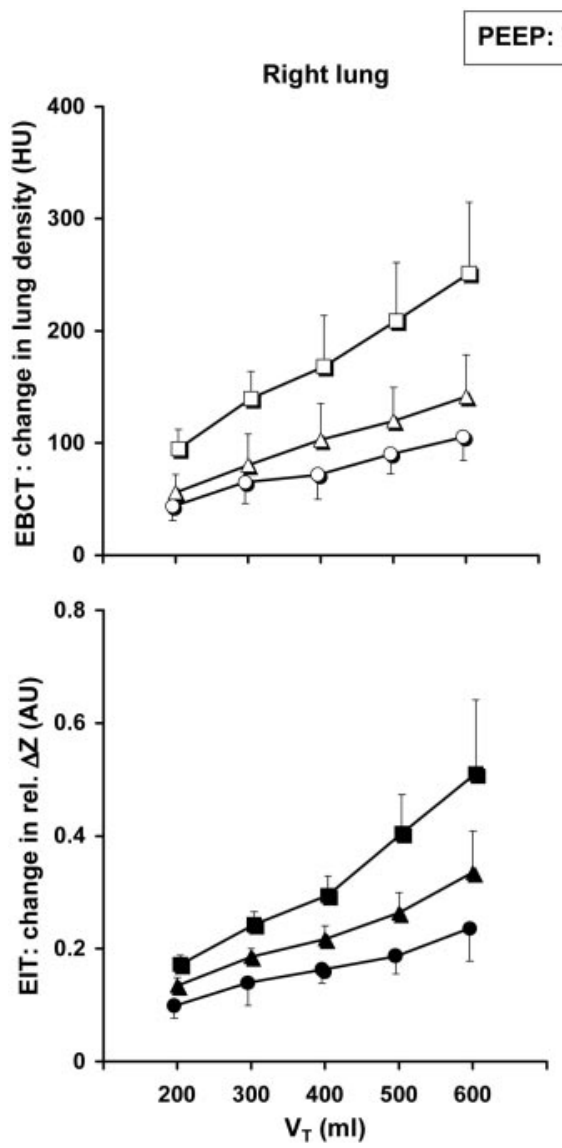

Left lung
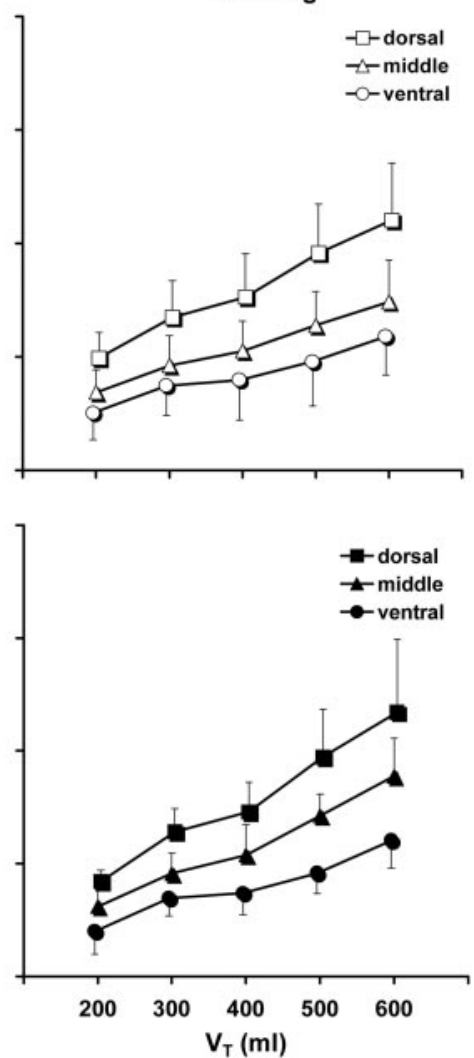

Fig. 4. Change in local lung air content dependent on VT at a PEEP of $7 \mathrm{cmH}_{2} \mathrm{O}$ at 6 ROIs, determined by EBCT (top) and EIT (bottom), with respect to the average lung air content during ventilation at $\mathrm{VT}=200 \mathrm{ml}$ and $\mathrm{PEEP}=2 \mathrm{cmH}_{2} \mathrm{O}$. Values are means $\pm \mathrm{SD}$. dorsal lung regions $(R=0.93$ and 0.92 , see Fig. 6 , top $)$ and the worst in the ventral lung region of the left lung $(R=0.75$, see Fig. 6 , bottom right $)$.

\section{DISCUSSION}

The results of the present study confirm the ability of EIT to identify local changes in lung air content. Topographically inhomogeneous changes of the local lung air content were elicited in artificially ventilated pigs by variation of the ventilatory pattern (14) and quantified by EIT in the dependent, intermediate, and nondependent regions of both lungs. The regional changes in lung air content determined by EIT were compared with the findings obtained by the reference EBCT technique. A good correspondence between the EIT and EBCT data was found, indicating that EIT will also be able to follow the changes in local air content in clinical settings.

Evaluation of EIT and EBCT scans. The comparison of the EIT and EBCT data was a challenging methodological aspect of the present study. Although both methods provide cross-sectional scans of the chest, the measuring principle, the characteristics of the collected data, as well as the data-acquisition rate and duration of the measurements are quite different. The EIT scans reflect the electrical properties of the chest tissues and are more suitable for functional than anatomic imaging (4). The EBCT scans show the distribution of the tissue density in the chest and provide primarily mor- phological data. The EIT time resolution is better, and the space resolution worse, than EBCT. Despite the mentioned differences between these two techniques, both the dielectric properties of lung tissue (20) and the lung density (3) sensed by EIT and EBCT, respectively, are a function of gas volume. This means that both methods can be applied to determine local changes in lung air content under in vivo conditions and, moreover, that the data obtained by EIT and EBCT can be compared when adequate evaluation procedures that take into account the differences between these techniques are chosen.

One of the important differences between the EIT and EBCT measurements relevant for data evaluation is the following: the beam of X-rays passing through the animal during EBCT scanning stays, to a large extent, confined to a single, two-dimensional plane, whereas the electrical currents used during EIT examinations leave the plane defined by the attached electrodes and travel through the tissues lying a few centimeters above and below the transverse plane studied. This means that the electrical impedance of the out-ofplane structures contributes to the collected EIT data, although with less sensitivity than the in-plane tissues. Thus the two-dimensional EIT scans represent the distribution of electrical impedance in a not ideal three-dimensional slice (e.g., Refs. 10, 22). Because of this phenomenon, the multislice scanning mode was chosen during the EBCT measurements, enabling an 
Fig. 5. Change in local lung air content dependent on VT at a PEEP of $12 \mathrm{cmH}_{2} \mathrm{O}$ at 6 ROIs, determined by EBCT (top) and EIT (bottom), with respect to the average lung air content during ventilation at $\mathrm{VT}_{\mathrm{T}}=200 \mathrm{ml}$ and PEEP $=2 \mathrm{cmH}_{2} \mathrm{O}$. Values are means \pm SD.
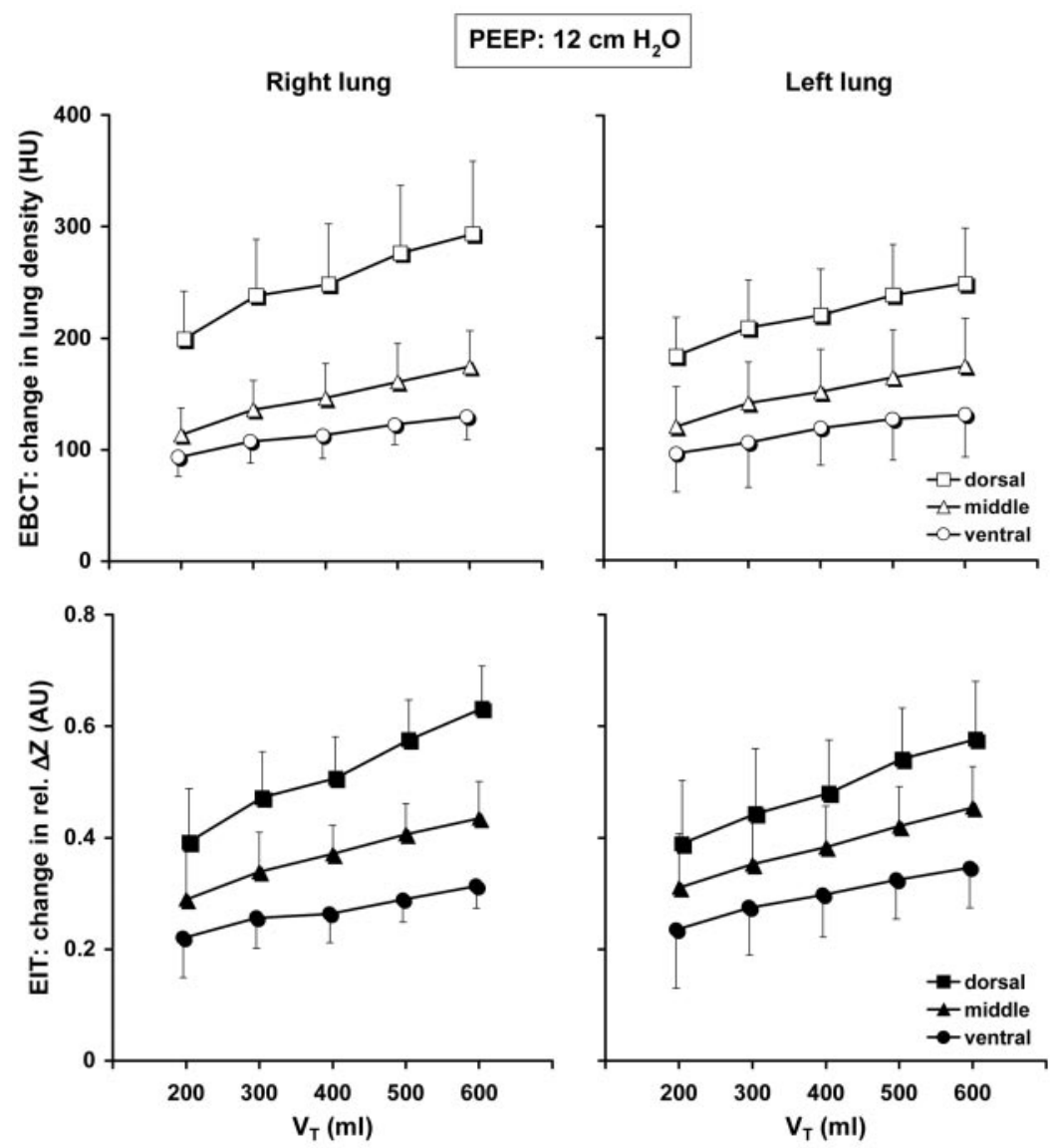

acquisition of four transverse slices spanning an almost 4-cm-broad transverse section of the chest, and an off-line averaging of the data obtained in these four slices was performed. In this way, a better correspondence between the EIT and EBCT data was secured, because the data originated from almost the same chest tissues.

Both EIT and EBCT enable an acquisition of series of scans. This was essential for proper selection of ROIs and elimination of possible sources of evaluation errors; however, during off-line data evaluation, the sets of EIT and EBCT scans were averaged over the whole respective scanning periods. In our laboratory's previous studies, the EIT data were analyzed in terms of, e.g., the magnitude of the local end-inspiratory-to-endexpiratory impedance differences (characterizing the local VT) or the local end-expiratory impedance values (representing the local functional residual capacity) (5, 6). A similar analysis had not been chosen in the present study, as it would have been imprecise in the case of the EBCT data. Because of the lower maximum EBCT scan rate and shorter scan period compared with EIT, the identification of the end-inspiratory and endexpiratory data points would have been problematic. Therefore, we have decided to use the above-mentioned less sophisticated data evaluation approach of data averaging in the present experimental series, which, however, guaranteed a reliable comparison between the EIT and EBCT data, as far as the detection of local changes in lung air content was concerned.

$E I T$ vs. EBCT. A good correlation between the lung air content changes determined by EIT and EBCT was found. The best correlation between the data obtained by these two techniques was found in the dependent lung regions. The worsening of the correlation observed in the middle and nondependent regions is a consequence of the chest (and lung) movement, which increases in direction toward the unsuspended ventral chest structures. This movement artifact plays no important role in EIT scans, because the size of the EIT images is not influenced by the ventilatory movements of the chest. Both the application of the excitation currents and the measurement of potential differences take place at surface electrodes placed on the chest circumference, and the electrodes copy the movements of the chest. In contrast with EIT, during EBCT examinations, neither the target rings around which the focused electron beam is scanned nor the detectors are in motion, and, consequently, the chest dimensions increase with inspiration and decrease with expiration in the corresponding scans. This dissimilarity of the effect of ventral lung movement on the acquired EIT and EBCT scans during inflation is not present during the simultaneously occurring caudal lung movement, which affects the EIT and EBCT scans in a similar way. 

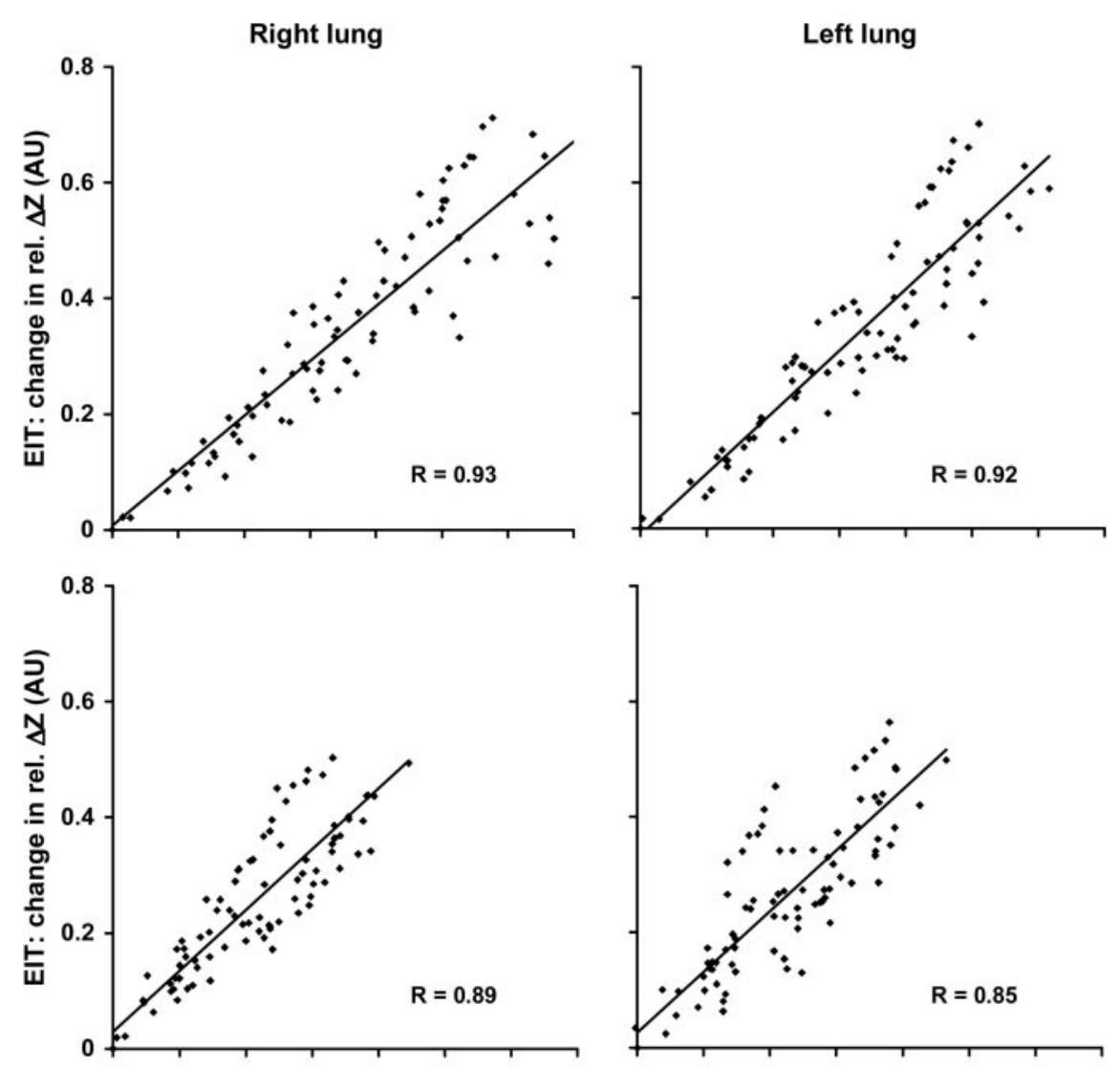

Fig. 6. Correlation between the lung air content changes determined by EBCT and EIT at the dorsal (top), middle (middle), and ventral (bottom) lung regions of the right and left lungs. $R$, correlation coefficient.
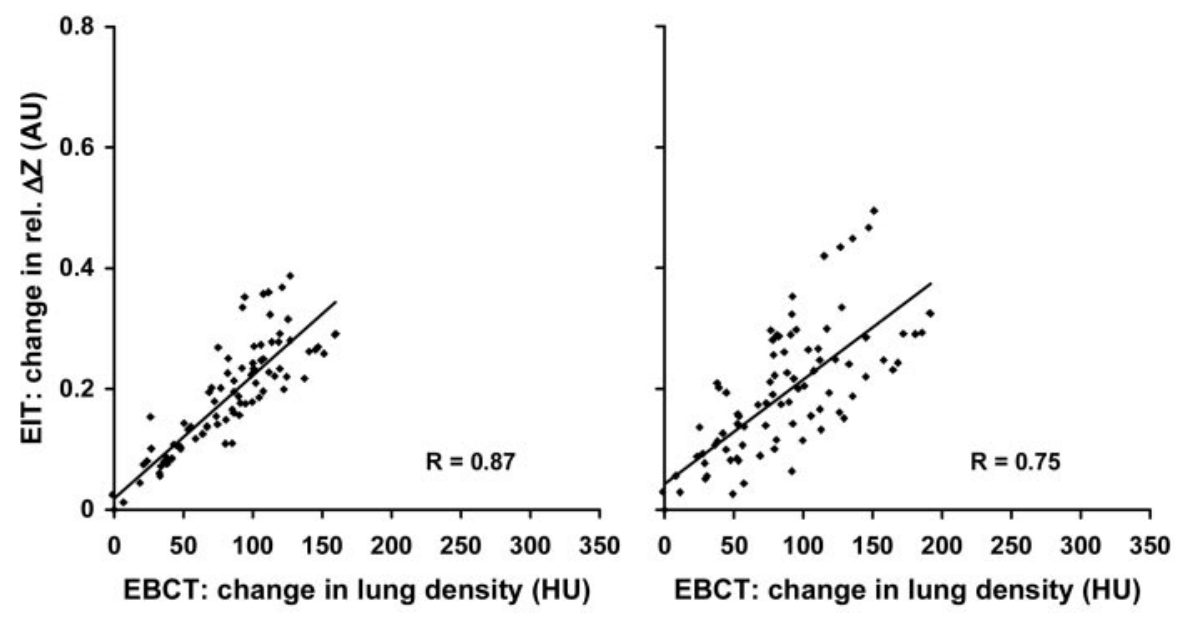

The worse correlation between the EIT and EBCT data found in the ventral ROI in the left lung is attributable not only to the above-mentioned effect of the anterior ventilatory movements but also to the cardiac action (see the corresponding ROI location in Fig. 2). The observed vertical gradient in local correlation coefficients is, in part, also the consequence of the almost twice as large changes in lung air content in the dependent regions compared with the nondependent ones. (See Fig. 6. The maximum lung density change observed in the dependent lung areas was $335 \mathrm{HU}$, whereas the maximum change of $190 \mathrm{HU}$ was found in the nondependent regions.)
The good agreement between the EIT and EBCT data is the major finding of our study. It confirms the results of previous experiments aimed at proving the ability of EIT to determine lung gas volume changes correctly. In those studies, the EIT data were acquired during stepwise inflation of lungs, and the resulting impedance changes were compared with global lung volume changes determined by spirometry $(10,13)$. However, in view of the known inhomogeneity of the lung function and the intention to use EIT in regional lung function monitoring, it was essential to compare the EIT data with an established method that provided data on the local, and not only global, lung ventilation. 
Our study is the first one to validate the EIT performance in identifying local changes in lung air content by using a generally accepted EBCT method as a reference. Until now, there existed only two other studies in which methods providing information on certain local aspects of the lung function were used to assess the quality of EIT findings. In those studies, the EIT data were compared with the technique of regional lung staining, which was used as a reference method for localizing lung ventilation defects (10) and singlephoton-emission computed tomography to quantify local ventilation magnitude (15).

The present experiments also proved that EIT correctly determined local changes in lung air content associated with modified ventilator settings. The findings regarding the effects of $\mathrm{V}_{\mathrm{T}}$ and PEEP correspond with the current physiological knowledge on the distribution of lung volumes and ventilation under conditions of general anesthesia and artificial ventilation obtained by other examination techniques, like ventilation scintigraphy (24) or computed tomography (8, $18,21,23)$, or by determination of lung mechanics (17, 19). This is an essential result if EIT is intended to be used in regional lung function monitoring in ventilated patients.

Conclusion. EIT is an emerging imaging technique that has the potential of becoming a useful, noninvasive bedside monitoring method in clinical settings. So far, EIT has not been routinely used in this environment, mainly because of the functional limitations of the EIT systems used. Thanks to the improved performance of the modern EIT devices (12) and the newly developed data evaluation procedures $(5,7,10,25)$, an increasing interest in this method can be observed in the recent years. The present results support the future use of adequate EIT technology, e.g., in the intensive care unit environment for estimating regional lung function in artificially ventilated patients. It is expected that functional EIT examinations will provide the clinically relevant information useful for the rapid adjustment of ventilatory settings and the optimization of ventilatory strategies.

The authors thank C. Ottersbach for technical assistance.

This study was supported by the German Aerospace Center and German Ministry for Education and Research Grant 50 TK 9804.

\section{REFERENCES}

1. Barber DC. A review of image reconstruction techniques for electrical impedance tomography. Med Phys 16: 162-169, 1989.

2. Becker CR, Schätzl M, Schoepg UJ, Brüning R, and Reiser MF. Technical bases and acquisition conditions of electron-beam computed tomography. Radiologe 38: 987-992, 1998.

3. Drummond GB. Computed tomography and pulmonary measurements. Br J Anaesth 80: 665-671, 1998.

4. Frerichs I. Electrical impedance tomography (EIT) in applications related to lung and ventilation: a review of experimental and clinical activities. Physiol Meas 21: R1-R21, 2000.

5. Frerichs I, Dudykevych T, Hinz J, Bodenstein M, Hahn G, and Hellige G. Gravity effects on regional lung ventilation determined by functional EIT during parabolic flights. J Appl Physiol 91: 39-50, 2001.
6. Frerichs I, Hahn G, and Hellige G. Thoracic electrical impedance tomographic measurements during volume controlled ventilation-effects of tidal volume and positive end-expiratory pressure. IEEE Trans Med Imaging 18: 764-773, 1999.

7. Frerichs I, Schiffmann H, Hahn G, and Hellige G. Noninvasive radiation-free monitoring of regional lung ventilation in critically ill infants. Intensive Care Med 27: 1385-1394, 2001.

8. Gattinoni L, Pelosi P, Crotti S, and Valenza F. Effects of positive end-expiratory pressure on regional distribution of tidal volume and recruitment in adult respiratory distress syndrome. Am J Respir Crit Care Med 151: 1807-1814, 1995.

9. Geddes LA and Baker LE. The specific resistance of biological material-a compendium of data for the biomedical engineer and physiologist. Med Biol Eng 5: 271-293, 1967.

10. Hahn G, Hartung C, and Hellige G. Elektrische Impedanztomographie (EIT) als Methode zur regionalen Beurteilung der Lungenventilation. Mainz, Germany: Akademie der Wissenschaften und der Literatur, 1996, p. 62-66.

11. Hahn G, Šipinková I, Baisch F, and Hellige G. Changes in the thoracic impedance distribution under different ventilatory conditions. Physiol Meas 16: A161-A173, 1995.

12. Hahn G, Thiel F, Dudykevych T, Frerichs I, Gersing E, and Hellige G. Quantitative evaluation of the performance of different electrical tomography devices. Biomed Tech (Berl) 46: 91-95, 2001.

13. Harris ND, Suggett AJ, Barber DC, and Brown BH. Applications of applied potential tomography (APT) in respiratory medicine. Clin Phys Physiol Meas 8, Suppl A: 155-165, 1987.

14. Hedenstierna G, Santesson J, and Baehrendtz J. Variations of regional lung function in acute respiratory failure and during anaesthesia. Intensive Care Med 10: 169-177, 1984.

15. Hinz J, Neumann P, Hahn G, Maripuu E, Andersson LG, Hellige G, Burchardi H, and Hedenstierna G. Electrical impedance tomography measures ventilation distribution: a comparison with ventilation scintigraphy (Abstract). Intensive Care Med 26, Suppl 3: 292, 2000.

16. Kunst PWA, de Vries PMJM, Postmus PE, and Bakker J. Evaluation of electrical impedance tomography in the measurement of PEEP-induced changes in lung volume. Chest 115: 1102-1106, 1999.

17. Liu JM, De Robertis E, Blomquist S, Dahm PL, Svantesson C, and Jonson B. Elastic pressure-volume curves of the respiratory system reveal a high tendency to lung collapse in young pigs. Intensive Care Med 25: 1140-1146, 1999.

18. Millar AB and Denison DM. Vertical gradients of lung density in healthy supine men. Thorax 44: 485-490, 1989.

19. Mundie TG, Easa D, Finn KC, Stevens EL, Hashiro G, and Balaraman V. Effect of baseline lung compliance on the subsequent response to positive end-expiratory pressure in ventilated piglets with normal lungs. Crit Care Med 22: 1631-1638, 1994.

20. Nopp P, Rapp E, Pfützner H, Nakesch H, and Ruhsam CH. Dielectric properties of lung tissue as a function of air content. Phys Med Biol 38: 699-716, 1993.

21. Puybasset L, Gusman P, Muller JC, Cluzel P, Coriat P, Rouby JJ, and the CT Scan ARDS Study Group. Regional distribution of gas and tissue in acute respiratory distress syndrome. III. Consequences for the effects of positive end-expiratory pressure. Intensive Care Med 26: 1215-1227, 2000.

22. Rabbani KS and Kabir AMBH. Studies on the effect of the third dimension on a two-dimensional electrical impedance tomography system. Clin Physiol 12: 393-402, 1991.

23. Reber A, Engberg G, Sporre B, Kviele L, Rothen HU, Wegenius G, Nylund $U$, and Hedenstierna G. Volumetric analysis of aeration in the lungs during general anaesthesia. $\mathrm{Br} J$ Anaesth 76: 760-766, 1996.

24. Rehder K, Sessler AD, and Rodarte JR. Regional intrapulmonary gas distribution in awake and anaesthetized-paralyzed man. J Appl Physiol 42: 391-402, 1977.

25. Smallwood RH, Hampshire AR, Brown BH, Primhak RA, Marven S, and Nopp P. A comparison of neonatal and adult lung impedances derived from EIT images. Physiol Meas 20: 401-413, 1999. 


\title{
Regional Ventilation by Electrical Impedance Tomography*
}

\section{A Comparison With Ventilation Scintigraphy in Pigs}

\author{
José Hinz, MD; Peter Neumann, MD, PhD; Taras Dudykevych, DIng; \\ Lars Goran Andersson, MD; Hermann Wrigge, MD; \\ Hilmar Burchardi, MD, PhD; and Goran Hedenstierna, MD, PhD, FCCP
}

\begin{abstract}
Study objective: The validation of electrical impedance tomography (EIT) for measuring regional ventilation distribution by comparing it with single photon emission CT (SPECT) scanning. Design: Randomized, prospective animal study.

Settings: Animal laboratories and nuclear medicine laboratories at a university hospital.

Participants: Twelve anesthetized and mechanically ventilated pigs.

Interventions: Lung injury was induced by central venous injection of oleic acid. Then pigs were randomized to pressure-controlled mechanical ventilation, airway pressure-release ventilation, or spontaneous breathing.

Measurements and results: Ventilation distribution was assessed by EIT using cross-sectional electrotomographic measurements of the thorax, and simultaneously by single SPECT scanning with the inhalation of ${ }^{99 \mathrm{~m}} \mathrm{Tc}$-labeled carbon particles. For both methods, the evaluation of ventilation distribution was performed in the same transverse slice that was approximately $4 \mathrm{~cm}$ in thickness. The transverse slice then was divided into 20 coronal segments (going from the sternum to the spine). We compared the percentage of ventilation in each segment, normalized to the entire ventilation in the observed slice. Our data showed an excellent linear correlation between the ventilation distribution measured by SPECT scanning and EIT according to the following equation: $y=0.82 x+0.7\left(R^{2}=0.92\right.$; range, 0.86 to 0.97$)$.

Conclusion: Based on these data, EIT seems to allow, at least in comparable states of lung injury, real-time monitoring of regional ventilation distribution at the bedside.
\end{abstract}

(CHEST 2003; 124:314-322)

Key words: regional ventilation; thoracic electrical impedance tomography

Abbreviations: $\quad$ APRV $=$ airway pressure release ventilation; $\quad \mathrm{CPAP}=$ continuous positive airway pressure; $\mathrm{EIT}=$ electrical $\quad$ impedance $\quad$ tomography; f-EIT $=$ functional electrical impedance tomography; EVLWI = extravascular lung water index; $\mathrm{FIO}_{2}=$ fraction of inspired oxygen; $\mathrm{PCV}=$ pressure-controlled ventilation; SPECT $=$ single photon emission CT; Vreg $=$ registered volume; VT $=$ tidal volume

$\mathbf{E}$ lectrical impedance tomography (EIT), which was developed in the early 1980s by Barber and Brown, ${ }^{1}$ generates cross-sectional images of the impedance distribution within a measured object. The basic principle of EIT is based on an alternatecurrent injection and voltage measurement via sur-

\footnotetext{
*From the Department of Anesthesiology, Emergency, and Intensive Care Medicine (Drs. Hinz, Neumann, Dudykevych, and Burchardi), University of Göttingen, Göttingen, Germany; the Department of Clinical Physiology (Drs. Andersson and Hedenstierna), University of Uppsala, Uppsala, Sweden; and the Department of Anesthesiology and Intensive Care Medicine (Dr. Wrigge), University of Bonn, Bonn, Germany.

This study was supported by grants from the Swedish Medical and Technical Research Councils (5315 and 299-2000-688), the Swedish Heart and Lung Foundation, and departmental funds. Manuscript received July 11, 2002; revision accepted January 29 , 2003.
}

face electrodes. If surface electrodes are placed around the thorax, changes in the electrical impedance during ventilatory maneuvers parallel to changes of aeration within the lungs can be measured. This enables the measurement of regional ventilation. EIT has been used increasingly as an experimental, noninvasive, lung-imaging technique. $^{2-14}$ EIT has been shown to detect physiologic events related to anatomic settings. ${ }^{2,5,8,15}$ Validation of the technique by an established clinical method,

Reproduction of this article is prohibited without written permission from the American College of Chest Physicians (e-mail: permissions@chestnet.org).

Correspondence to: José Hinz, MD, Department of Anesthesiology, Emergency, and Intensive Care Medicine, Georg-AugustUniversity, Robert-Koch-Str 40, D-37075 Göttingen, Germany; e-mail:mail@josehinz.de 
however, has not been obtained. The aim of this study was therefore to validate the measurement of regional ventilation, assessed by a newly developed high-performance EIT device, with ventilation scintigraphy. Since EIT may have the potential to be a bedside technique in the intensive care setting, we performed the tests in a porcine lung damage model, during different ventilatory support modes.

\section{MATERIALS AND Methods}

\section{Study Protocol}

After approval by the local animal ethics committee, 12 pigs of mixed breed (ie, Hampshire, Yorkshire, and Swedish country breed) [mean $( \pm \mathrm{SD})$ weight, $30 \pm 4 \mathrm{~kg}$ ] were anesthetized and mechanically ventilated. Acute lung injury was induced in all pigs by IV injections of oleic acid (Apoteksbolaget; Göteborg, Sweden) that was suspended in $20 \mathrm{~mL}$ isotonic saline solution. The dosing of the oleic acid was guided by intermittently taken arterial blood samples in order to achieve a $\mathrm{PaO}_{2} /$ fraction of inspired oxygen $\left(\mathrm{FIO}_{2}\right)$ ratio of about $200 \mathrm{~mm} \mathrm{Hg}$. After the induction of lung injury, a stabilization period of at least $2 \mathrm{~h}$ was allowed before we proceeded with the experimental protocol, so that lung injury was stable, as assessed by oxygenation and respiratory mechanics. Another hour later, the pigs were transferred to the nuclear medicine laboratories, the animals were placed in the supine position, with the front legs stretched cranially, and simultaneous measurements of regional ventilation distribution using EIT $^{16}$ and single photon emission CT (SPECT) scanning ${ }^{17}$ were performed. The ventilation distribution was assessed in an approximately $4 \mathrm{~cm}$-thick transverse slice of the lung. The slice then was divided into 20 equally thick coronal segments going from the sternum to the spine. For both meth- ods, the percentage of ventilation in each coronal segment was calculated and compared, as shown in Figure 1.

The investigation was performed in the experimental laboratories of the Department of Clinical Physiology at the University Hospital of Uppsala, Sweden.

\section{Anesthesia}

Azaperone (Stresnil; Janssen Pharmaceutica; Beerse, Belgium), $40 \mathrm{mg}$ IM, were administered as premedication. General anesthesia was induced with remifentanil (Ultiva; Glaxo Wellcome; Hamburg, Germany) [0.04 mg/kg/min] and ketamine (Ketanest; Parke-Davis; Berlin, Germany), followed by a constant IV infusion of both drugs to maintain either controlled ventilation or spontaneous breathing. The animals received tracheotomies and were either spontaneously breathing or mechanically ventilated through a cuffed tube. Prior to measurements, 1,000 mL Ringer-acetate (Pharmacia AB; Stockholm, Sweden) at body temperature was infused.

\section{Ventilation}

Mechanical ventilation was provided with a ventilator (EVITA 4; Dräger; Lübeck, Germany). Respirator settings were initially in the pressure-controlled mode with a peak airway pressure of $15 \mathrm{~cm}_{2} \mathrm{O}$, a positive end-expiratory pressure of $5 \mathrm{~cm} \mathrm{H}_{2} \mathrm{O}$, a respiratory rate of 20 breaths/min, an inspiratory/expiratory ratio of $1: 2$, and an $\mathrm{FiO}_{2}$ of 0.5 .

After oleic acid administration and a stabilization period of approximately $2 \mathrm{~h}$, the pigs were randomized to pressurecontrolled ventilation (PCV) without spontaneous breathing, PCV with spontaneous breathing (ie, airway pressure release ventilation $[\mathrm{APRV}]$ ), or spontaneous breathing (ie, continuous positive airway pressure [CPAP]). The pigs also were studied for other purposes, which are reported on elsewhere. Another hour later, the pigs were moved to the gamma camera for the study, keeping the ventilator settings constant in the PCV group, and

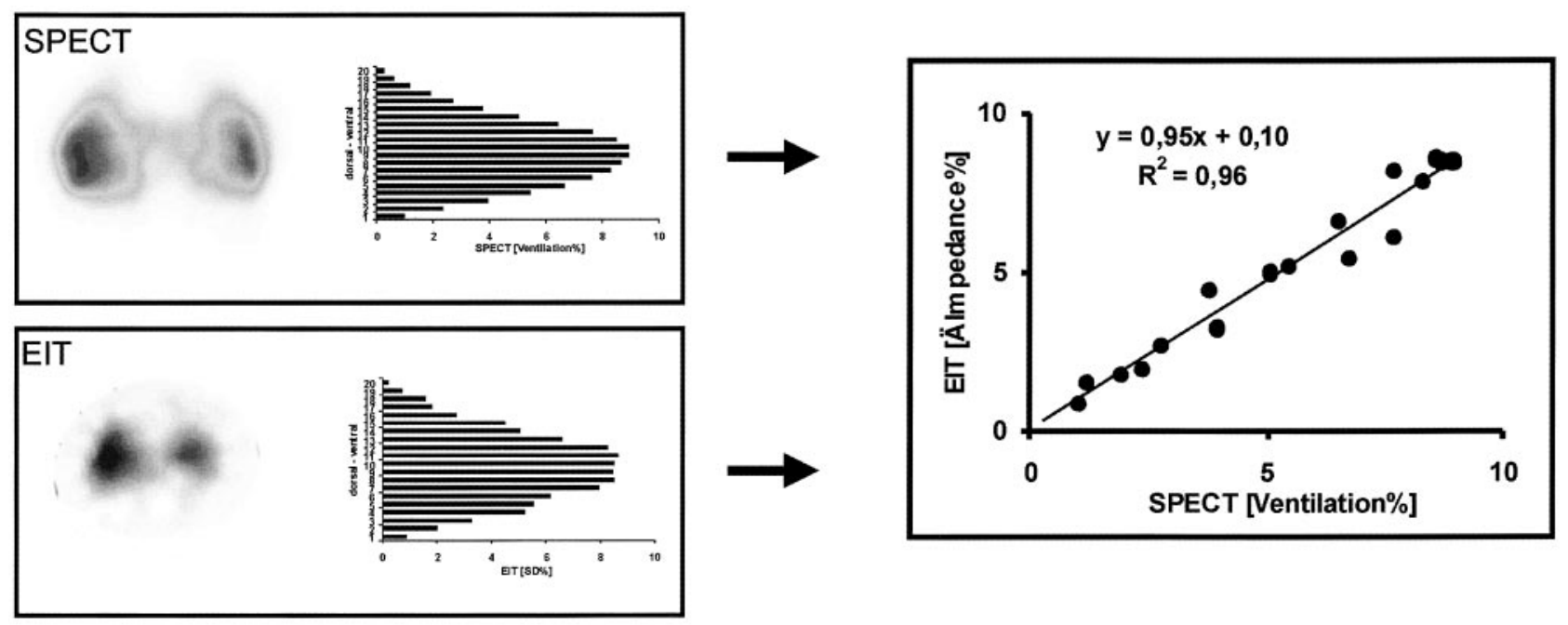

FigURE 1. Analysis procedure. For each pig, simultaneous transversal measurements from SPECT scanning and EIT were performed in a 4-cm slice of the thorax. Each slice was divided into 20 anterior-posterior segments (going from the sternum to the spine). Ventilation for both methods was calculated by the percentages of either regional minute volume for SPECT scanning or by the percentages of regional impedance change for EIT. The correlation of ventilation in each segment was calculated. $\ddot{\mathrm{A}}=\Delta$. 
adjusting the ventilator support in the APRV and CPAP groups so that spontaneous breathing was resumed. Arterial blood gas samples were analyzed (ABL 300 and OSM 3 Hemoximeter; Radiometer; Copenhagen, Denmark).

\section{Hemodynamics}

For arterial blood gas sampling, an 18-gauge catheter was inserted into the carotid artery, together with a thermistor-tipped fiberoptic catheter (Pulsiocath 4F FT PV 2024; Pulsion Medical System; Munich, Germany), which was advanced into the descending aorta for measurements of extravascular lung water index (EVLWI). EVLWI was calculated automatically (Pulsion COLD Z-021; Pulsion Medical System) after injecting 8 to $10 \mathrm{~mL}$ $1 \mathrm{mg} / \mathrm{mL}$ indocyanine green (ICG-Pulsion; Pulsion Medical System), which was mixed in sterile water (temperature range, $5^{\circ} \mathrm{C}$ to $7^{\circ} \mathrm{C}$ ) randomly within the respiratory cycles. ${ }^{18,19}$ The mean of triplicate measurements was used.

\section{Compliance}

Respiratory compliance was measured during controlled mechanical ventilation before and after the induction of acute lung injury. Airway pressure (Huba Control; Würenlos, Switzerland) was measured at the y-piece of the respirator. Tidal volume (VT) was calculated from gas flow measured by pneumotachography (flow head: Fleisch No. 2; Fleisch; Lausanne, Switzerland; differential pressure transducer: Huba Control). Data were sampled at $200 \mathrm{~Hz}$ and were stored for offline evaluation on a personal computer. Compliance was calculated according to the equation VT/(Pei - Pee), where Pei is the end-inspiratory pressure, and Pee is the end-expiratory pressure. Compliance was averaged over a 3-min recording period.

EIT

An applied potential tomography system (Goe-MF; EITGroup Göttingen; Göttingen, Germany) was used for electrical impedance measurements. Sixteen commercially available radiograph transparent surface electrodes (Blue sensor BR-50-K; Medicotest A/S; Olstyke, Denmark) were placed around the thorax of the pigs at the level of the xiphoid process. For data collection, an alternating current $(5 \mathrm{~mA} \mathrm{p}-\mathrm{p}, 50 \mathrm{kHz}$ ) was injected between one pair of adjacent electrodes. The resulting surface potentials were measured between the remaining adjacent electrode pairs. All 16 adjacent electrode pairs were used as the injecting electrodes, one pair after the other, with the surface potential being measured with the remaining electrodes. One data collection cycle was completed when all pairs of adjacent electrodes had been used once as injecting electrodes. The sampling rate of the used EIT device was 13 cycles per second, and 1,000 cycles were measured during each observation period. Thus, the mean acquisition time was about $77 \mathrm{~s}$, corresponding to approximately 35 to 60 respiratory cycles, depending on the applied respiratory mode. According to the method described by Barber and Brown, ${ }^{20}$ the resulting 208 surface potentials were normalized to the mean surface potential during the measurement period, and the normalized surface potentials were used subsequently for the reconstruction of regional impedance changes by a back-projection. Functional EIT $^{16}$ (f-EIT) was performed to determine the local lung impedance change as an indicator for regional ventilation. The generation of f-EIT is based on the assessment of the amplitude of impedance changes in every region of an EIT image. An example of an f-EIT image is shown in Figure 1.

For further evaluation, this f-EIT image then was divided into
20 equally thick coronal segments going from the sternum to the spine. The impedance change in each segment as well as in the whole EIT image was calculated. Ventilation in each segment then was calculated as a percentage of the summarized change in impedance in each segment divided by the summarized impedance change of the whole EIT image. The data were stored for off-line evaluation on a personal computer.

\section{SPECT Scanning}

Ventilation distribution was assessed by SPECT scanning using ${ }^{99 \mathrm{~m}}$ Tc-labeled carbon particles (Technegas; Tetley Medical Limited; Lucas Heights, NSW, Australia). The particle size is approximately $0.1 \mu \mathrm{m}$, and its distribution in the lung has been shown to be similar to that of radioactive gas. ${ }^{17}$ This "pseudogas" was injected continuously during mechanical ventilation over a period of 3 to $5 \mathrm{~min}$ from a 2 -L syringe that was connected to the Y-piece. During the injection of the carbon particles, a pressure of approximately $31 \mathrm{~cm} \mathrm{H}_{2} \mathrm{O}$ was maintained within the syringe, as controlled by a manometer connected to the syringe via a three-way stopcock, resulting into a continuous flow of carbon particles into the Y-piece during the whole respiratory cycles. Images were acquired on a dual-head gamma camera (Maxxus; General Electric Systems; Milwaukee, WI) that was equipped with all-purpose low-energy collimators. The SPECT acquisitions were performed in 64 projections (32 projections per head) and were stored in a $64 \times 64$ matrix. The acquisition time was $15 \mathrm{~s}$ for each projection.

The data were reconstructed on a computer workstation (HERMES; Nuclear Diagnostics; Stockholm, Sweden). The acquired data were prefiltered with a two-dimensional Butterworth filter (cutoff frequency, 0.14; filter order, 10). Filtered backprojection reconstruction was performed without applying attenuation correction. After corrections for the background, the number of counts was measured in each volume element of the lungs.

Minute ventilation was assessed by pneumotachography (as described above). Ventilation in the $4 \mathrm{~cm}$-thick slice near the EIT electrodes was calculated as the number of counts within the slice, times minute ventilation, divided by the total number of counts within the whole lung. By adding coronal planes, the slices were divided into 20 equally thick segments in the ventral-dorsal direction going from the sternum to the spine. The ventilation of each segment finally was calculated as the fractional activity in each segment times minute ventilation.

Since animals with varying degrees of pulmonary edema and damage were studied, the risk of carbon particles being trapped in the edematous airways was considered to be possible. The intrapulmonary distributions of the carbon particles therefore were compared with a reference technique, the inhalation of radioactive krypton gas, in four pigs. ${ }^{81 \mathrm{~m}} \mathrm{Kr}$ was produced as a gas in a ${ }^{81} \mathrm{Rb} /{ }^{81 \mathrm{~m}} \mathrm{Kr}$ generator (KryptoScan; Mallinckrodt Medical BV; Petten, the Netherlands). The krypton was continuously administered directly into the ventilator during the whole acquisition time. The scintigraphic acquisition of carbon particles and ${ }^{81 \mathrm{~m}} \mathrm{Kr}$ was performed simultaneously in two separate windows of $140 \mathrm{keV}( \pm 10 \%)$ and $186 \mathrm{keV}( \pm 10 \%)$, respectively, for technetium and krypton. Except for an extension of the acquisition time per projection to $35 \mathrm{~s}$, the same camera and acquisition settings were used as described above. After correction for crossover into the $140-\mathrm{keV}$ window, reconstruction was performed as described above.

\section{Statistical Analysis}

Calculations were performed with a statistical software package (Statistica, version 5.1; StatSoft Inc; Tulsa, OK) on a personal 
computer (Pentium II $233 \mathrm{MHz}$ processor; Intel; Santa Clara, CA; and Windows 95; Microsoft; Redmond, WA). All data are presented as the minimum to maximum (median). A p value of $<0.05$ was chosen as the level of significance.

The Pearson correlation analysis was used to calculate the correlation between the ventilation distribution assessed by the two methods. In addition, EIT and SPECT data were compared using the statistical analysis method of Bland and Altman. ${ }^{21}$ Bias was defined by the mean differences between EIT and SPECT, and it represented the systematic error. The SD of the mean differences is considered to represent the random error variability between the two techniques. Bias \pm 2 SDs was the limit of agreement of both methods.

\section{RESULTS}

Four pigs each were ventilated in the PCV, APRV, and CPAP modes, and the median values of the $\mathrm{PaO}_{2} / \mathrm{FIO}_{2}$ ratio were 176,197 , and $212 \mathrm{~mm} \mathrm{Hg}$, respectively. Respirator settings, blood gas data, and respiratory compliance are summarized in Table 1.

\section{Correlation of Inhaled Krypton and Nebulized Carbon Particles}

A good correlation between the distributions of inhaled krypton and nebulized carbon particles was obtained, according to the following equation: VregTc $=0.97 \times \operatorname{VregKr}+4.3 \quad\left(R^{2}=0.98 ;\right.$ mean Vreg, approximately $350 \mathrm{~mL}$ ), where Vreg is registered volume, Tc is technetium, and $\mathrm{Kr}$ is krypton. This was considered proof that the carbon particles could be used for the subsequent comparisons between EIT and SPECT could also be use in the present pigs with oleic acid-induced pulmonary edema.

\section{Pooled Data for Regional Ventilation Distribution}

A highly significant linear correlation between regional ventilation measured by EIT and SPECT scanning was found, according to the equation $\mathrm{y}=0.82 \mathrm{x}+0.73\left(R^{2}=0.92 ;\right.$ range, 0.86 to 0.97$)[\mathrm{Fig}$ $2]$. However, the regression coefficient was $<1.0$ $(p<0.001)$, and we found a positive intercept $(\mathrm{p}<0.001)$.

As can be seen from a Bland-Altman analysis, EIT tended to overestimate ventilation in regions that were poorly ventilated and to underestimate ventilation in well-ventilated regions (Fig 3). Thus, the magnitude of the regional ventilation affected the correlation between EIT and SPECT scanning values. However, the difference between EIT and SPECT scanning did not exceed $10 \%$ and was mostly much less than that.

\section{Influence of Spontaneous and Mechanical Ventilation}

The same correlation between EIT and SPECT data was seen whether the pigs were receiving continuous mechanical ventilation (ie, PCV) or were breathing spontaneously (ie, CPAP). A combination of mechanical and spontaneous ventilation (approximate ratio, 80:20) [ie, APRV] also resulted in a similar correlation (Fig 4). Thus, the respiratory mode had no effect on the relationship between EIT and SPECT scanning. Figure 5 shows the spatial ventilation distribution in the dorsoventral orientation (going from the sternum to the spine) of the different ventilatory modes as examined with EIT and SPECT scanning.

\section{Influence of Lung Edema, Minute Ventilation, and $\mathrm{PaCO}_{2}$}

Since the distribution of the oleic acid-induced edema is gravity-dependent, ${ }^{22}$ dorsal lung regions are more severely affected than anterior regions. Therefore, an analysis was made about whether this (ie, the upper, healthier lung regions vs the lower, sicker lung regions) had an effect on the relationship between EIT and SPECT scanning, but no such effect was found (Fig 6). Moreover, the compliance of the respiratory system as well as of the EVLWI were compared with the correspondence between EIT and SPECT scanning. No correlation was found between compliance or ELVWI, either in absolute numbers or in terms of the relative change from baseline, and the difference between EIT and SPECT scanning.

Minute ventilation and $\mathrm{PaCO}_{2}$ varied considerably between pigs, with several pigs displaying hypoventilation as a consequence of the oleic acid-induced lung damage and the use of spontaneous breathing modes (Table 1). This may have been due to increased dead space ventilation during spontaneous breathing, and it necessitated an analysis of the influence of minute ventilation and $\mathrm{PaCO}_{2}$ on the performance of EIT. However, no correlation between minute ventilation, or $\mathrm{PaCO}_{2}$, and the difference between EIT and SPECT scanning was seen. Thus, the accuracy of measuring regional ventilation by EIT was not affected by the degree of ventilation.

\section{Discussion}

EIT is a noninvasive technique with the potential to monitor regional ventilation distribution. Since its introduction, the hardware and software have been improved continuously. ${ }^{23,24}$ Hahn and coworkers ${ }^{25}$ developed more advanced algorithms for the analysis of dynamic physiologic phenomena with low amplitudes than was initially supplied (Mark I or DAS01P; Royal Hallamshire Hospital; Sheffield, UK). Thus, they introduced the f-EIT and the averaging technique. ${ }^{16}$ These improvements, together with 


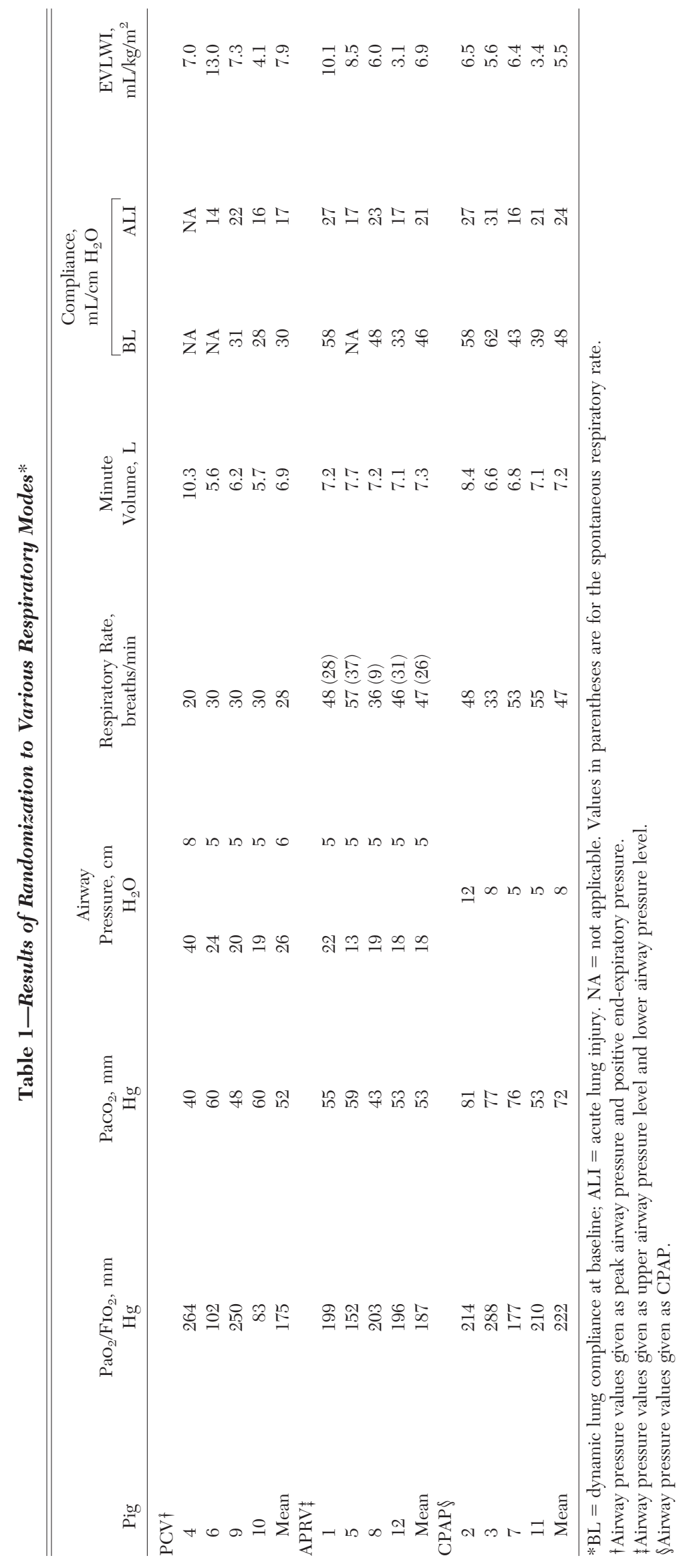




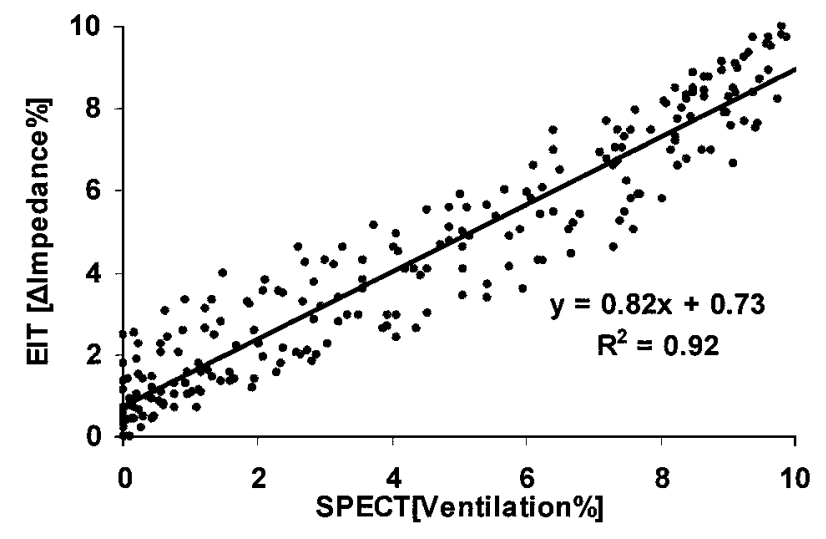

Figure 2. Linear correlation. Comparison of regional lung ventilation measured with EIT and SPECT scanning in 240 regions of interests in 12 pigs. Values given as the percentage of ventilation assessed by EIT and SPECT scanning.

other refinements of the technique, have resulted in a higher cycle rate and a better signal-to-noise ratio, which enable the system to monitor impedance changes during for example respiration. The major advantages of EIT are thereby that it is noninvasive, it is easy to use at the bedside, and data collection can be performed with a high time resolution.

\section{EIT vs SPECT}

The validation of the EIT technique has so far been based on correlation analysis with global parameters or on the reproduction of known physiologic phenomena or anatomic settings. ${ }^{2-5,11,15,16}$ A partitioning of ventilation between the right and left lung by EIT and a comparison with radionuclide scanning was performed by Kunst and coworkers. ${ }^{12}$ However, they needed to average 10 data collection

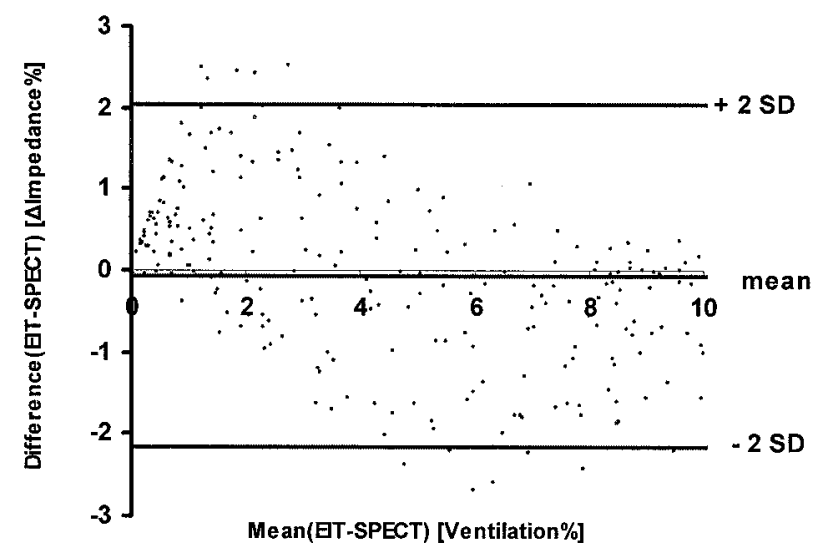

Figure 3. Bland-Altman analysis. Comparison of regional lung ventilation measured with EIT and SPECT scanning in 240 regions of interests in 12 pigs. Values given as the percentage of ventilation assessed by EIT and SPECT scanning.
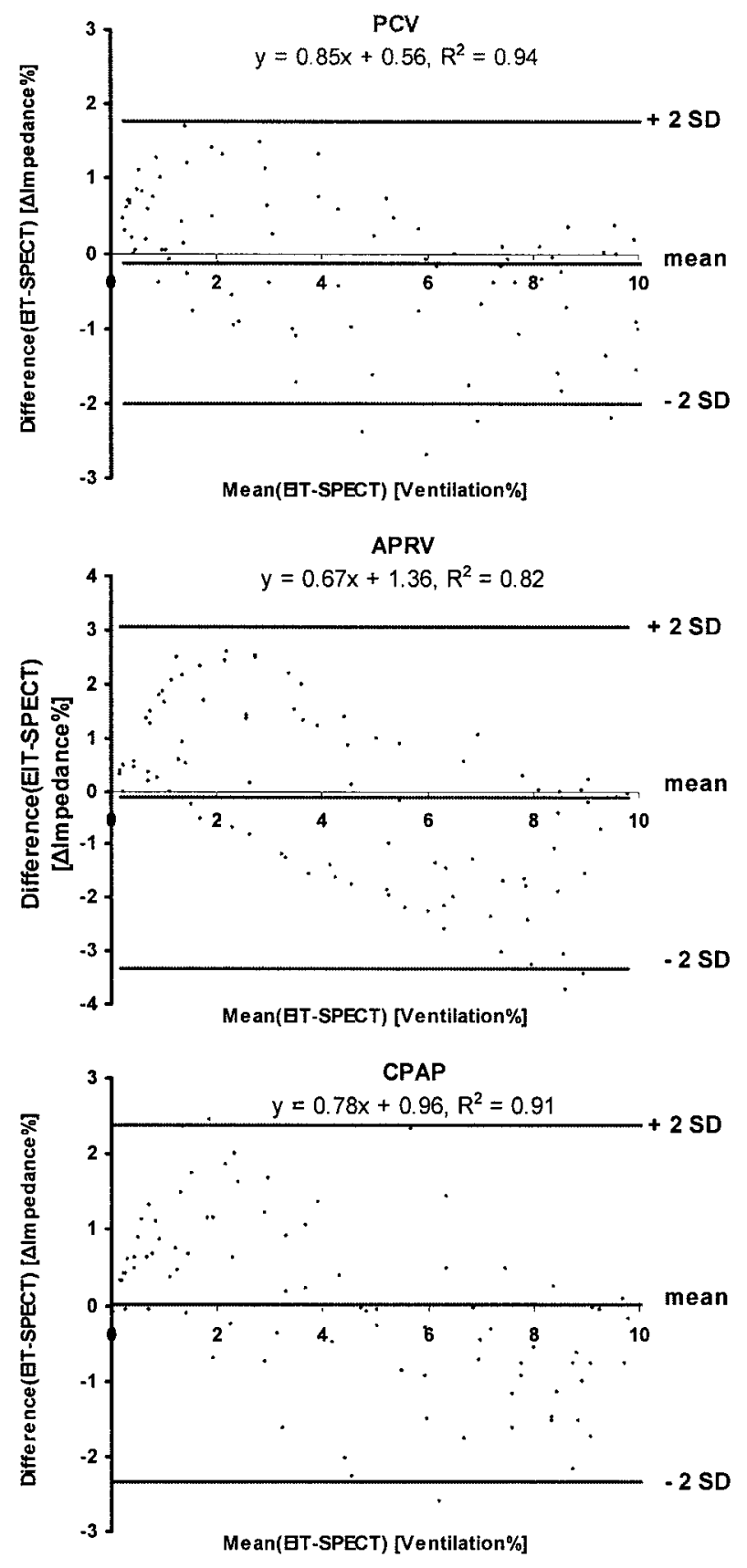

Figure 4. Bland-Altman analysis. Separate analysis of regional lung ventilation from pigs that were breathing spontaneously (ie, CPAP), that were mechanically ventilated (ie, $\mathrm{PCV}$ ), and that breathed APRV. Eighty regions of interests were measured in four pigs. The linear correlation is given in the plot. Values given as the percentage of ventilation assessed by EIT and SPECT scanning.

cycles to obtain one EIT image. Although the time resolution was rather poor, they found a good correlation between the EIT and radionuclide methods for separate lungs. With the advanced hardware and software ${ }^{9,25}$ used in the present study, the technique also should be suitable for the regional analysis of 


\section{SPECT}
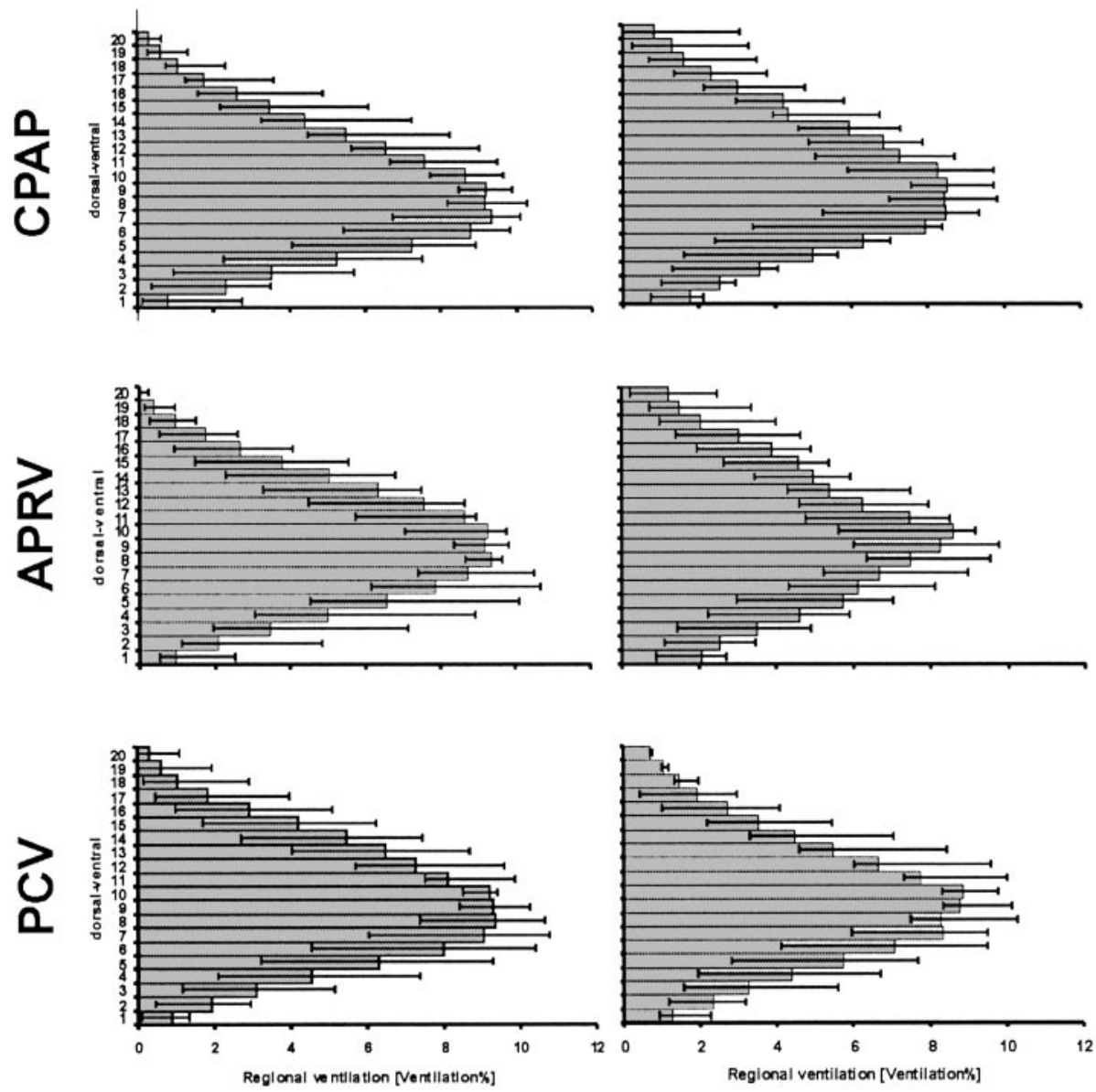

FIGURE 5. Comparison of ventilation distributions in different ventilatory modes in dorsal-to-ventral orientation of a transversal plane at the processus xiphoideus measured by SPECT scanning and EIT. Twelve pigs with oleic acid-induced lung injury were studied. Four pigs were mechanically ventilated (ie, PCV), four pigs were mechanically ventilated with spontaneous breathing (ie, APRV), and four pigs breathed spontaneously (ie, CPAP).

ventilation within the lungs. We obtained an excellent linear correlation between f-EIT and ventilation scintigraphy (ie, SPECT scanning). Whether breathing was spontaneous or mechanically delivered did not affect the correlation. Moreover, we did not see any influence of the severity of lung damage in this correlation on a global level (ie, no correlation to respiratory compliance and EVLWI) and on a regional level (ie, no difference in the relationship between EIT and SPECT in dependent, more severely affected lung regions or upper, nondependent regions).

However, a regression coefficient of 0.82 and the positive intercept between SPECT scanning (independent variable) and EIT (dependent variable) showed that EIT slightly underestimated ventilation compared to SPECT scanning in rather wellventilated regions and overestimated it in poorly ventilated areas. The difference in regional ventilation as measured by both methods was $<10 \%$, and this is comparable to the coefficient of variation of most physiologic methods. The EIT method thus seems to be acceptable as a monitoring device in, for example, the intensive care setting. Potential causes of the measured differences between EIT and SPECT are discussed in the following paragraph.

\section{Linearity of EIT}

Holder and Khan ${ }^{26}$ determined the linear operating range of the impedance change caused by various polyacrylamide gels with different impedance char- 

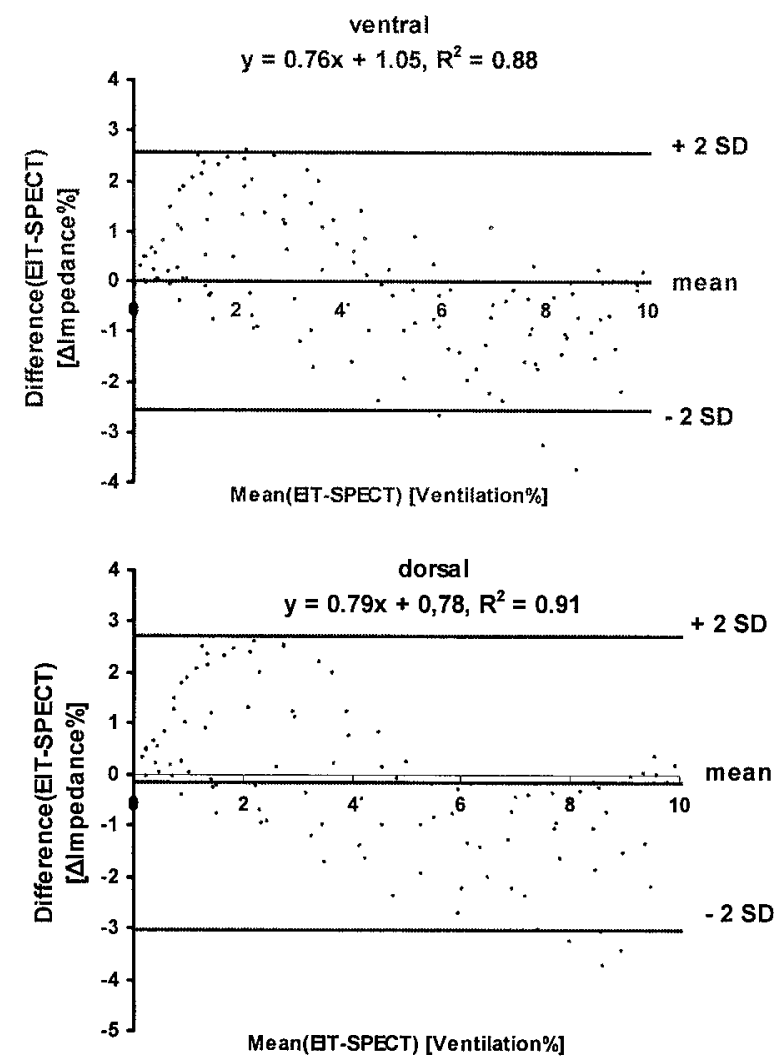

Figure 6. Bland-Altman analysis. Separate analyses of dorsal and ventral regions from 12 pigs were made. Lung ventilation in 120 regions of interests in 12 pigs was measured. The linear correlation is given in the plot. Values given as the percentage of ventilation assessed by EIT and SPECT scanning.

acteristics. As a physiologic model, they used a saline solution-filled tank and an EIT system (APT System Mark I; IBEES; Sheffield, UK). Impedance changes increased in a linear fashion up to $20 \%$. Impedance changes of $>20 \%$ were underestimated by the EIT system. This effect may depend on the electrical properties of the EIT system ${ }^{27}$ or on the image reconstruction algorithm, ${ }^{28}$ which is the same as the one that we have used. The algorithm for image reconstruction is based on the assumptions that the object is two-dimensional and circular. Furthermore, the distribution of resistivity initially has to be uniform, the changes in resistivity should be small, and the electrodes should be spaced equally around the thorax. Nevertheless, EIT images can be acquired even if these assumptions are violated. The respiratory patterns in the present study included VT values $\leq 15 \mathrm{~mL} / \mathrm{kg}$, which confined impedance changes to a maximum of $25 \%$. This may be the reason for the underestimation of larger regional volume changes, as seen in the present study.

\section{Possibilities and Limitations of EIT}

Value of Information: Although we calculated only 20 regions of interest from one f-EIT image, the spatial resolution allows the calculation of a maximum of 912 regions of interest per image. According to Hahn et al, ${ }^{29}$ the minimal detectable lung volume by EIT is in the range of 9 to $29 \mathrm{~mL}$, whereas the spatial resolution using SPECT scanning is approximately $1 \mathrm{~mL}$. Thus, regional ventilation of wellventilated lung areas, which are located close to poorly or nonventilated lung regions, may be underestimated by EIT and vice versa. By measuring EIT over time, this spatial resolution combined with the time resolution of $\leq 44 \mathrm{~Hz}$ offers the possibility of following regional ventilation. A lower sampling rate of $1 \mathrm{~Hz}$ is sufficient to monitor slow events like stepwise changes in lung volume during breathholding procedures. ${ }^{30}$ Higher sampling rates may be suitable for rapid physiologic events, like a forced expiration in spontaneously breathing subjects.

Since EIT images are generated by relatively small local impedance changes, whereas absolute impedance values may vary considerably among different subjects, EIT is most suitable for intraindividual monitoring, with each subject serving as his own reference.

Clinical Application: As it is noninvasive and easily transportable, EIT might be suitable for monitoring regional lung function in mechanically ventilated patients at the bedside. It may be used to acquire information, such as regional lung ventilation, regional distribution of VT, regional lung volume, and functional residual capacity. ${ }^{31}$ Furthermore, fluid accumulation, redistribution of ventilation inside the lungs, ${ }^{32}$ and regional compliance curves ${ }^{11}$ may be reconstructed from EIT data. Thus, EIT could be used, for example, to monitor atelectasis and hyperinflation during different ventilatory strategies or in opening procedures. In patients with COPD, EIT measurement of regional lung function during therapeutic interventions such as bronchodilator administration or noninvasive ventilation also might be possible. In neonates, for whom radiation exposure is undesirable, EIT potentially may replace radiographic investigations in certain situations. ${ }^{7}$ However, EIT should not be applied to obtain morphologic information similar to CT scanning or MRI, since the latter diagnostic methods provide anatomic information with a much higher spatial and morphologic resolution compared to EIT.

\section{CONCLUSION}

EIT data of regional ventilation correlated closely to ventilation distribution by SPECT scanning in a porcine, oleic acid-lung damage model. Whether breathing was spontaneous or mechanical had no effect on the relationship between EIT and SPECT scanning, and the severity of lung damage on a global 
or a regional level did not influence the relationship either. A slight overestimation of ventilation in wellventilated areas and a slight underestimation in poorly ventilated areas was seen, but the difference was always $<10 \%$ of ventilation measured by SPECT scanning. Thus, EIT appears to be an interesting monitoring technique for use in the intensive care setting.

\section{REFERENCES}

1 Barber DC, Brown BH. Applied potential tomography. J Phys [E] 1984; 17:723-733

2 Adler A, Amyot R, Guardo R, et al. Monitoring changes in lung air and liquid volumes with electrical impedance tomography. J Appl Physiol 1997; 83:1762-1767

3 Frerichs I, Hahn G, Golisch W, et al. Monitoring perioperative changes in distribution of pulmonary ventilation by functional electrical impedance tomography. Acta Anaesthesiol Scand 1998; 42:721-726

4 Frerichs I, Hahn G, Hellige G. Gravity-dependent phenomena in lung ventilation determined by functional EIT. Physiol Meas 1996; 17(suppl):A149-A157

5 Frerichs I, Hahn G, Hellige G. Thoracic electrical impedance tomographic measurements during volume controlled ventilation-effects of tidal volume and positive end-expiratory pressure. IEEE Trans Med Imaging 1999; 18:764-773

6 Frerichs I, Hahn G, Hellige G. Bestimmung der regionalen Ventilation bei einseitiger Lungenschädigung mittels funktioneller elektrischer Impedanztomographie. Intensiv und Notfallbehandlung 1999; 24:87-92

7 Frerichs I, Hahn G, Schiffmann H, et al. Monitoring regional lung ventilation by functional electrical impedance tomography during assisted ventilation. Ann N Y Acad Sci 1999. 873:493-505

8 Frerichs I, Hahn G, Schroder T, et al. Electrical impedance tomography in monitoring experimental lung injury. Intensive Care Med 1998; 24:829-836

9 Hahn G, Frerichs I, Golisch W, et al. Determining local lung ventilation by functional electrical impedance tomography under clinical circumstances. Biomed Tech (Berl) 1997; 42 (Suppl):213-214

10 Hahn G, Frerichs I, Kleyer M, et al. Local mechanics of the lung tissue determined by functional EIT. Physiol Meas 1996; 17(suppl):A159-A166

11 Kunst PW, Bohm SH, de Vazquez A, et al. Regional pressure volume curves by electrical impedance tomography in a model of acute lung injury. Crit Care Med 2000; 28:178-183

12 Kunst PW, Vonk NA, Hoekstra OS, et al. Ventilation and perfusion imaging by electrical impedance tomography: a comparison with radionuclide scanning. Physiol Meas 1998; 19:481-490

13 Kunst PW, Vonk NA, Straver B, et al. Influences of lung parenchyma density and thoracic fluid on ventilatory EIT measurements. Physiol Meas 1998; 19:27-34

14 Kunst PW, de Vries PM, Postmus PE, et al. Evaluation of electrical impedance tomography in the measurement of PEEPinduced changes in lung volume. Chest 1999; 115:1102-1106
15 Adler A, Shinozuka N, Berthiaume Y, et al. Electrical impedance tomography can monitor dynamic hyperinflation in dogs. J Appl Physiol 1998; 84:726-732

16 Hahn G, Sipinkova I, Baisch F, et al. Changes in the thoracic impedance distribution under different ventilatory conditions. Physiol Meas 1995; 16(suppl):A161-A173

17 Burch WM, Sullivan PJ, Lomas FE, et al. Lung ventilation studies with technetium-99m Pseudogas. J Nucl Med 1986; $27: 842-846$

18 Lichtwarck-Aschoff M, Beale R, Pfeiffer UJ. Central venous pressure, pulmonary artery occlusion pressure, intrathoracic blood volume, and right ventricular end-diastolic volume as indicators of cardiac preload. J Crit Care 1996; 11:180-188

19 Lichtwarck-Aschoff M, Zeravik J, Pfeiffer UJ. Intrathoracic blood volume accurately reflects circulatory volume status in critically ill patients with mechanical ventilation. Intensive Care Med 1992; 18:142-147

20 Barber DC, Brown BH. Recent developments in applied potential tomography-APT. In: Bacharach SL, ed. Proceedings of the 9th Conference of Information Processing in Medical Imaging. Dordrecht, the Netherlands: Martinus Nijhoff, 1986; 106-121

21 Bland JM, Altman DG. Statistical methods for assessing agreement between two methods of clinical measurement. Lancet 1986; 1:307-310

22 Neumann P, Berglund JE, Mondejar EF, et al. Effect of different pressure levels on the dynamics of lung collapse and recruitment in oleic-acid-induced lung injury. Am J Respir Crit Care Med 1998; 158:1636-1643

23 Koukourlis CS, Kyriacou GA, Sahalos JN. A 32-electrode data collection system for electrical impedance tomography. IEEE Trans Biomed Eng 1995; 42:632-636

24 Li JH, Joppek C, Faust U. Fast EIT data acquisition system with active electrodes and its application to cardiac imaging. Physiol Meas 1996; 17(suppl):A25-A32

25 Hahn G, Beer M, Frerichs I, et al. A simple method to check the dynamic performance of electrical impedance tomography systems. Physiol Meas 2000; 21:53-60

26 Holder DS, Khan A. Use of polyacrylamide gels in a saline-filled tank to determine the linearity of the Sheffield Mark 1 electrical impedance tomography (EIT) system in measuring impedance disturbances. Physiol Meas 1994; 15(suppl):A45-A50

27 Brown BH, Seagar AD. The Sheffield data collection system. Clin Phys Physiol Meas 1987; 8:91-97

28 Barber DC. Quantification in impedance imaging. Clin Phys Physiol Meas 1990; 11(suppl):45-56

29 Hahn G, Hartung C, Hellige G. Bestimmung der grösse minimal erfassbarer areale mit ventilationsstörungen. In: Thews G, ed. Elektrische impedanztomographie (EIT) als methode zur regionalen beurteilung der lungenventilation. Mainz, Germany: Gustav Fischer Verlag, 1998; 77

30 Harris ND, Suggett AJ, Barber DC, et al. Applications of applied potential tomography (APT) in respiratory medicine. Clin Phys Physiol Meas 1987; 8(suppl):155-165

31 Hinz J, Hahn G, Neumann P, et al. End-expiratory lung impedance change enables bedside monitoring of endexpiratory lung volume change. Intensive Care Med 2003; 29:37-43

32 Hinz J, Hahn G, Sydow M, et al. Bed-side measurement of PEEP effects on ventilation distribution by f-EIT: preliminary results [abstract]. Intensive Care Med 1998; 24(suppl):66 


\section{J. Hinz \\ G. Hahn \\ P. Neumann \\ M. Sydow \\ P. Mohrenweiser \\ G. Hellige \\ H. Burchardi}

\section{End-expiratory lung impedance change enables bedside monitoring of end-expiratory lung volume change}

Received: 20 December 2001

Accepted: 11 October 2002

Published online: 20 November 2002

(C) Springer-Verlag 2002

This study was supported by departmental funding

\author{
J. Hinz ( $)$ - G. Hahn · P. Neumann \\ P. Mohrenweiser · G. Hellige \\ H. Burchardi \\ Department of Anaesthesiology, \\ Emergency and Intensive Care Medicine, \\ University of Göttingen, \\ Robert-Koch-Str. 40, \\ 37075 Göttingen, Germany \\ e-mail: mail@josehinz.de \\ Tel.: +49-551-398826 \\ Fax: +49-551-398676 \\ M. Sydow \\ Department of Anaesthesiology and \\ Intensive Care Medicine, \\ St. Johannes-Hospital Dortmund, Germany
}

Abstract Objective: The aim of the
study was to investigate the effect of
lung volume changes on end-expira-
tory lung impedance change (ELIC)
in mechanically ventilated patients,
since we hypothesized that ELIC may
be a suitable parameter to monitor
lung volume chance at the herside.
Design Studie II
quiring
Setting
versity
chanic:
cluded
tients v
trolled

respiratory rate. m oruer to muuce changes in the end-expiratory lung volume (EELV), PEEP levels were increased from 0 mbar to 5 mbar, 10 mbar, and 15 mbar. At each PEEP level EELV was measured by an open-circuit nitrogen washout manoeuvre and ELIC was measured simultaneously using Electrical Impedance Tomography (EIT) with sixteen electrodes placed on the circumference of the thorax and connected with an EIT device. Cross-sectional electro-tomographic measurements of the thorax were performed at each PEEP level, and a modified Sheffield backprojection was used to reconstruct images of the lung impedance. ELIC was calculated as the average of the end-expiratory lung impedance change. Results: Increasing PEEP stepwise from 0 mbar to 15 mbar resulted in an linear increase of EELV and ELIC according to the equation: $\mathrm{y}=0.98 \times$ $-0.68, r^{2}=0.95$. Conclusion: EIT is a simple bedside technique which enables monitor lung volume changes during ventilatory manoeuvres such as PEEP changes.

Keywords Thoracic electrical impedance tomography .

End-expiratory lung impedance change $\cdot$ End-expiratory lung volume
Abbreviations $A R F$ acute respiratory failure $\cdot \mathrm{CO}_{2}$ carbon dioxide $\cdot$ EIT Electrical Impedance Tomography $\cdot E E L V$ end-expiratory lung volume $\cdot \mathrm{FiO}_{2}$ inspiratory oxygen concentration - PEEP positive end-expiratory pressure $\cdot N_{2}$ nitrogen . $\mathrm{O}_{2}$ oxygen

\section{Introduction}

The monitoring of end-expiratory lung volume (EELV) could be an important tool to assess the pulmonary status and the effects of ventilator settings in critically ill patients [1]. The use of positive end-expiratory pressure (PEEP) is considered part of the standard therapy for patients with acute respiratory failure (ARF) requiring mechanical ventilation [2]. PEEP improves arterial oxygenation due to alveolar recruitment and is thereby associated with an increase in EELV. Consequently, techniques for the bedside measurement of EELV are desirable, but not yet available.

Information of alveolar recruitment can be obtained from CT scanning [3], but this is not a bedside method. 
Table 1 Patient characteristics

\begin{tabular}{|c|c|c|c|c|c|c|c|}
\hline Patient & $\begin{array}{l}\text { Age } \\
\text { (years) }\end{array}$ & $\begin{array}{l}\text { Weight } \\
(\mathrm{kg})\end{array}$ & $\begin{array}{l}\text { Height } \\
(\mathrm{cm})\end{array}$ & Diagnosis & $\begin{array}{l}\text { Lung } \\
\text { diagnosis }\end{array}$ & $\begin{array}{l}\text { Lung injury } \\
\text { score }\end{array}$ & $\mathrm{FiO}_{2}$ \\
\hline 1 & 37 & 80 & 178 & Skull brain injury & Atelectasis & 1.0 & 0.40 \\
\hline 3 & 30 & 80 & 182 & Skull brain injury & Atelectasis & 1.3 & 0.55 \\
\hline 4 & 78 & 60 & 167 & & Pneumonia & 3.0 & 0.60 \\
\hline 5 & 62 & 90 & 180 & $\begin{array}{l}\text { Subarachnoid } \\
\text { hemorrhage }\end{array}$ & ARDS & 3.3 & 0.60 \\
\hline 8 & 69 & 115 & 185 & & ARDS & 2.3 & 0.40 \\
\hline 9 & 61 & 85 & 175 & & Pneumonia & 3.0 & 0.45 \\
\hline 10 & 51 & 92 & 183 & & Pneumonia & 2.0 & 0.40 \\
\hline Mean \pm SD & $58 \pm 16$ & $81 \pm 16$ & $175 \pm 7$ & & & $2.0 \pm 0.9$ & \\
\hline
\end{tabular}

Another approach to measure alveolar recruitment is an open-circuit multibreath nitrogen-washout manoeuvre, first described by Darling et al. in 1940 [4].

Electrical Impedance Tomography was developed in 1984 by Barber and Brown [5]. EIT generates cross-sectional images of the impedance distribution within a transverse slice of the thorax. The basic principle of EIT is based on an alternate current injection and voltage measurement via surface electrodes placed around the thorax. Since the electrical properties of the chest varies depending on variations in the air content, ventilationinduced impedance changes can be measured by an EIT device [6]. Consequently, this method offers the possibility of continuously measuring lung volume changes at the bedside.

The aim of this study was to investigate the effects of lung volume changes induced by different PEEP levels on electrical impedance tomography and to compare changes in electrical impedance with changes in the EELV as measured by the multibreath nitrogen-washout manoeuvre.

\section{Patients and methods}

Study protocol

After approval by the local ethics committee, the investigation was performed in the intensive care unit of the Department of Anaesthesiology, Emergency and Intensive Care Medicine, University Hospital Göttingen, Germany.

Informed written consent to participate in this study was obtained from the nearest relatives of ten mechanically ventilated patients (mean \pm standard deviation: age $58 \pm 16$ years, weight $81 \pm 16 \mathrm{~kg}$, lung injury score $2 \pm 0.9$ [7], days on mechanically ventilation $7 \pm 3$ days) were included into the study (for patient characteristics see Table 1).

Inclusion criteria were:

- Mechanically ventilated patient with acute respiratory failure $\left(\mathrm{PaO}_{2} / \mathrm{FiO}_{2}<300 \mathrm{mmHg}\right)$.

- Mechanical ventilation $>24 \mathrm{~h}$ before onset of the study.

- Age $\geq 18$ years.
- Informed written consent of the next kin.

- Clinically indicated arterial blood pressure measurement.

Exclusion criteria were:

- Inappropriate inclusion criteria.

- Pregnancy.

- Terminal illness.

- Unstable hemodynamics.

All patients were ventilated using an Evita2 ventilator (Dräger, Lübeck, Germany) in a volume-controlled mode with constant flow and a respiratory rate of $10 \cdot \mathrm{min}^{-1}$ ). Tidal volume, inspiratory oxygen concentration and the level of positive end-expiratory pressure (PEEP) were set by the attending physician in order to achieve normocapnia $\left(\mathrm{PaCO}_{2} 35-45 \mathrm{mmHg}\right)$ and an oxyhemoglobin saturation $\left(\mathrm{SaO}_{2}\right)>95 \%$. At the beginning of the study the PEEP level was decreased to $0 \mathrm{cmH}_{2} \mathrm{O}$ and occasionally the $\mathrm{FiO}_{2}$ was increased so that the $\mathrm{SaO}_{2}$ remained above $90 \%$. Thereafter, PEEP was increased stepwise to 5 mbar, 10 mbar, and 15 mbar after intervals of approximately 40 min when a steady state condition had been achieved. EIT measurements were performed continuously during the total observation period. At the end of each study period end-expiratory lung volume (EELV) was measured by an open-circuit multibreath nitrogen-washout manoeuvre and simultaneously recording of the end-expiratory lung impedance change was performed by electrical impedance tomography (EIT). We investigated the change of EELV and end-expiratory lung impedance change against baseline values with PEEP 0 mbar.

Open-circuit multibreath nitrogen-washout manoeuvre

An open-circuit multibreath nitrogen-washout manoeuvre was performed in similar way to the method described by Darling et al. [4], with modifications which have been described in detail elsewhere [8]. Briefly, gasflow was measured with a pneumotachograph (Fleisch No. 2, Fleisch, Lausanne, Switzerland) and a differential pressure transducer (Huba Control, Würenlos, Switzerland) directly connected to a heat and moisture exchanger (Humid-Vent 2, Gibeck Respiration, Väsby, Sweden) at the endotracheal tube. Inspiratory gas and expiratory gas was continuously sampled via a capillary (length: $3.09 \mathrm{~m}$, flow: $1 \mathrm{ml} \cdot \mathrm{s}^{-1}$ ) connected to the y-piece of the breathing circuit. Nitrogen $\left(\mathrm{N}_{2}\right)$, oxygen $\left(\mathrm{O}_{2}\right)$, and carbon dioxide $\left(\mathrm{CO}_{2}\right)$ were measured by a mass spectrometer (MGA 1100, Perkin Elmer, Pomona, Calif., USA) which delivered gas concentrations as fractions excluding water vapour. All data were sampled online by an analogue/digital converter (DT2801-A, Data Translation, Marlboro, Mass., USA) at a rate of $40 \mathrm{~Hz}$ and processed by an IBM AT compatible personal computer. The data ac- 
quisition and processing software was programmed with a commercially available software program (Asyst 4.0, Keithley Asyst, Taunton, Mass., USA).

The flow measuring system was calibrated with a gas mixture of known gas concentrations $\left(65 \% \mathrm{~N}_{2}, 30 \% \mathrm{O}_{2}\right.$, and $\left.5 \% \mathrm{CO}_{2}\right)$ and definite viscosity using a precision calibration pump (Engström Megamed 05, Engström, Stockholm, Sweden) that generates a sinusoidal flow pattern. During calibration measurements the instantaneous gas viscosity was determined from the actual gas fraction in order to correct the measured flow signal for the changing gas composition as described by Brunner et al. [9]. Volume was then obtained from the corrected flow signal by offline analysis. To minimize the drift of the flow signal by an offset, the pressure transducer was meticulously adjusted during zero-flow conditions before each measurement.

\section{Determination of end-expiratory lung volume (EELV)}

Calculation of EELV was performed offline. The multibreath nitrogen-washout manoeuvre was started by changing the $\mathrm{FiO}_{2}$ at end-expiration from baseline to 1.0. Fractional nitrogen concentration $\left(\mathrm{FN}_{2}\right)$ at baseline was determined as average $\mathrm{N}_{2}$-concentration before the start of washout. The EELV calculation procedure was started at end-expiration with the first oxygen washin breath. As the first breath usually still contains a certain amount of $\mathrm{N}_{2}$, this inspired $\mathrm{N}_{2}$-volume was subtracted from the cumulative $\mathrm{N}_{2}$-volume calculated from the washout procedure. To reduce the influence of signal noise to $\mathrm{N}_{2}$ washed out, direct calculation from the measurement was finished at $3 \%$ of the baseline $\mathrm{FN}_{2}$. Additionally, a correction for tissue $\mathrm{N}_{2}$ as described by Cournand et al. [10] was done in all patients. EELV were calculated from the measured $\mathrm{N}_{2}$ volume divided by $\mathrm{N}_{2}$ at baseline. For further details of determination and accuracy of the multibreath nitrogenwashout manoeuvre see [8].

\section{Electrical Impedance Tomography}

An Applied Potential Tomography System (APT System MK1, IBEES, Sheffield, UK) was used for the electrical impedance measurements. Sixteen surface electrodes (Blue sensor BR-50-K, Medicotest A/S, Olstyke, Denmark) were placed around the thorax of the patients in one transversal plane corresponding to the 6th intercostal parasternal space. An alternating current $(5 \mathrm{~mA} \mathrm{p}-\mathrm{p}, 50 \mathrm{kHz})$ was injected between a pair of adjacent electrodes. The resulting surface potentials were measured between the remaining adjacent electrode pairs. In turns, all 16 adjacent electrode pairs were used as injecting electrodes. When all pairs of adjacent electrodes had been used as injecting electrodes, one data collection cycle consisting of 208 surface potential data was completed. We performed 1,000 data collection cycles per measurement with a sampling rate of 10 cycles per second. Thus, the mean acquisition time was $100 \mathrm{~s}$, corresponding to 14 respiratory cycles during mechanical ventilation. The surface potentials were used to compute a sequence of the cross-sectional distribution of impedance changes within the thorax using a modified Sheffield back-projection [11] for tomographic reconstruction of an EIT image. The back-projection generates images which show the impedance changes as compared to a reference dataset. This method, therefore, allows the visualization of physiological or pathological phenomena, which induce impedance changes [12]. As the reference dataset we used 1,000 data recording cycles at PEEP zero for each individual patient.

End-expiratory lung impedance change (ELIC)

For every EIT image mean relative impedance change was calculated. The sequence of EIT images over time was used to calculate

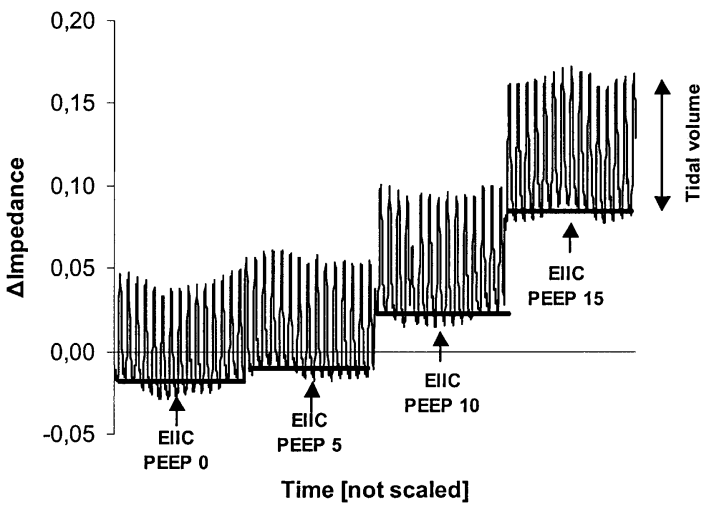

Fig. 1 Analysis of EIT measurement during mechanical ventilation with identical tidal volume at different PEEP-levels in one patient. The diagram shows four examples of the global time course of relative lung impedance change (impedance) at different PEEP levels. Each PEEP level was analysed for $100 \mathrm{~s}$, corresponding to 14 respiratory cycles. Measurements of the end-expiratory lung impedance change (end-expiratory lung impedance change) and end-expiratory lung volume were performed simultaneously

mean lung impedance time course (LITC) within the observed part of the thorax along the time. End-expiratory impedance change, indicating EELV, was subsequently calculated by averaging minimal LITC values of 14 consecutive breaths. An example of LITC is shown in Fig. 1. Data were stored for offline evaluation on a personal computer (Pentium II $233 \mathrm{MHz}$, Microsoft Windows 95).

\section{Hemodynamics and gas exchange}

Arterial blood sampling was performed via a 20-gauge catheter which was inserted in the radial or femoral artery for clinical reasons. Arterial blood gas samples were analyzed by ABL 300 and OSM 3 Hemoximeter (Radiometer, Copenhagen, Denmark).

ECG, systemic arterial, and central venous pressures were displayed on a bedside monitor together with the oxyhemoglobin saturation (Datex AS/3, Datex Divison Instrumentarium, Helsinki, Finland) and recorded with reference to atmospheric pressure at the mid-thoracic level at end-expiration.

\section{Statistics}

Calculations were performed using the STATISTICA software package (Statistica 5.1, StatSoft Inc, Tulsa, USA) on a personal computer (Pentium II $233 \mathrm{MHz}$, Microsoft Windows 95).

All data are presented as Min-Max (Median) unless stated otherwise. Since the data were not normally distributed, as tested by the Shapiro-Wilk W-test, we subsequently applied a Wilcoxon matched-pair test to test the significance between different endexpiratory lung volumes and end-expiratory lung impedance time courses against reference at PEEP 0 .

Linear regression analysis using the least square method was applied to correlate EELV and end-expiratory lung impedance change changes, respectively, against the different PEEP levels. Pearson's correlation analysis was used to detect any correlation between EELV and end-expiratory lung impedance change. In addition, EELV and end-expiratory lung impedance change data were compared using the statistical methods described by Bland and Altman [13], except for the bias which could not be compared due to the process of normalization. The standard deviation (SD) of the mean differences is considered to represent the random er- 


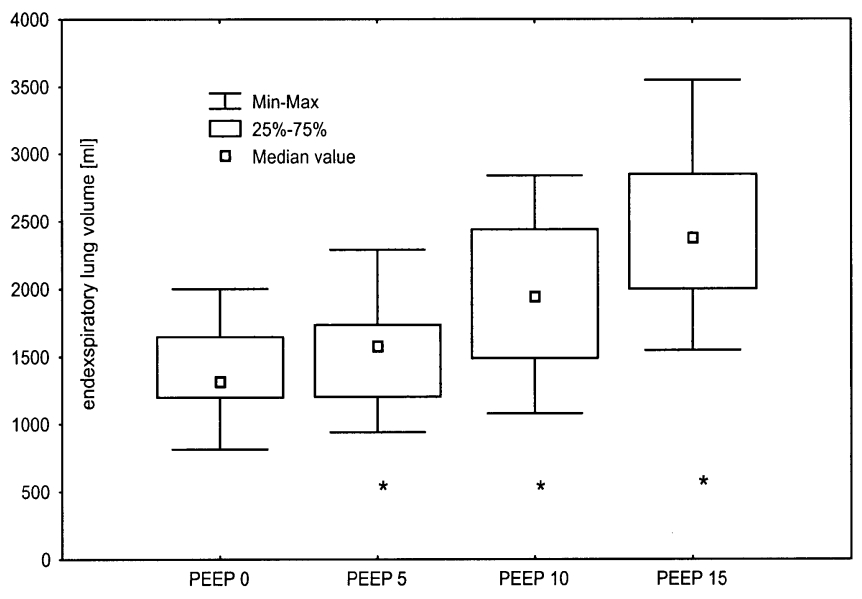

Fig. 2 Box-plots of end-expiratory lung volume at different PEEP-levels in ten mechanically ventilated patients. $* P<0.05$ against reference at PEEP $0 \mathrm{cmH}_{2} \mathrm{O}$

ror variability between both techniques. Mean difference $\pm 2 S D$ was the limit of agreement of both methods. For all statistical tests $P<0.05$ was considered to be significant.

\section{Results}

Variation of end-expiratory lung volume (EELV)

EELV increased significantly from 815-2002(1316) ml at $0 \mathrm{cmH}_{2} \mathrm{O}$ PEEP, to 940-2291(1578) ml with a PEEP of $5 \mathrm{cmH}_{2} \mathrm{O}$, to $1080-2841(1944) \mathrm{ml}$ with a PEEP of $10 \mathrm{cmH}_{2} \mathrm{O}$, and to 1546-3548(2379) $\mathrm{ml}$ with a PEEP of $15 \mathrm{cmH}_{2} \mathrm{O}$ (Min-Max(Median). All data are summarized in Fig. 2 and Table 2.

Variation of end-expiratory lung impedance change (ELIC)

According to our definition end-expiratory lung impedance change was $0.00-0.00(0.00)$ at a PEEP level of $0 \mathrm{cmH}_{2} \mathrm{O}$ (reference), it increased to $0.00-0.04(0.01)$

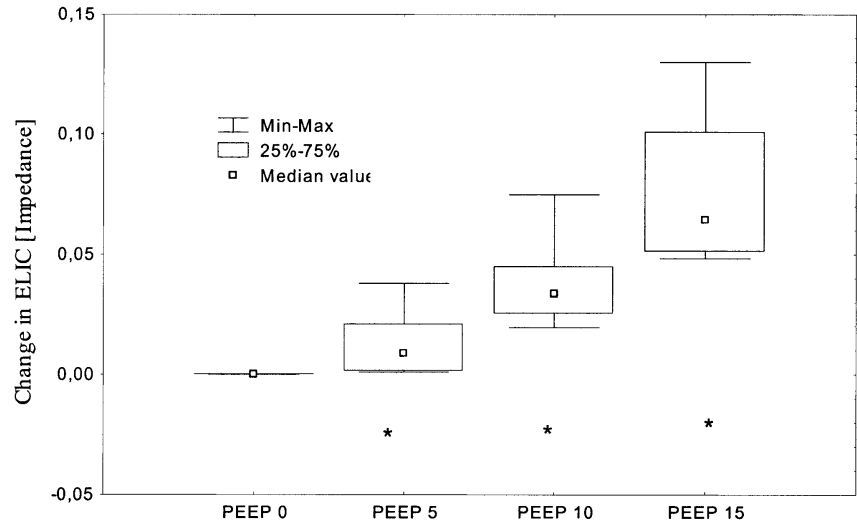

Fig. 3 Box-plots of change in end-expiratory lung impedance change (end-expiratory lung impedance change) at different PEEP levels in ten mechanically ventilated patients. $* P<0.05$ against reference at PEEP $0 \mathrm{cmH}_{2} \mathrm{O}$

with a PEEP of $5 \mathrm{cmH}_{2} \mathrm{O}$, increased further to 0.02 $0.08(0.03)$ at a PEEP level of $10 \mathrm{cmH}_{2} \mathrm{O}$, and reached a maximum value of $0.05-0.13(0.06)$ with a PEEP level of $15 \mathrm{cmH}_{2} \mathrm{O}$ [Min-Max(Median) of impedance change]. Similar to the changes of EELV, with each increase of PEEP a significant increase of end-expiratory lung impedance change was noted. All data are summarized in Fig. 3 and Table 3.

The long-term stability of the EIT signal, checked at PEEP 5 by comparison of the end-expiratory lung impedance change-values during the first minute, and after 10,20, 30 , and $40 \mathrm{~min}$, showed a variation of $1.5-6.1 \%(3.1 \%)$.

Correlation of end-expiratory lung volume (EELV) and end-expiratory lung impedance change (ELIC)

Increasing PEEP stepwise from 0 mbar to 15 mbar resulted in an linear increase of EELV and end-expiratory lung impedance change (overall $r^{2}=0.95$ ). The withinsubject $r^{2}$ were 0.96-0.99(0.99) [Min-Max (Median)]. The data are summarized in Fig. 4.
Table 2 End-expiratory lung volume at different PEEP-levels in ten mechanically ventilated patients. Data as range and median

\begin{tabular}{lllll}
\hline Patient & PEEP 0 $(\mathrm{ml})$ & PEEP 5 $(\mathrm{ml})$ & PEEP 10 $(\mathrm{ml})$ & PEEP 15 $(\mathrm{ml})$ \\
\hline 1 & 2002 & 2291 & 2764 & 3482 \\
2 & 1645 & 1736 & 2167 & 2574 \\
3 & 1291 & 1546 & 2003 & 2547 \\
4 & 1341 & 1720 & 2440 & 2849 \\
5 & 815 & 940 & 1080 & 3546 \\
6 & 1686 & 2035 & 2841 & 1573 \\
7 & 1028 & 1054 & 1310 & 2079 \\
8 & 1202 & 1206 & 1490 & 2000 \\
9 & 1375 & 1414 & 1725 & 2211 \\
10 & 1285 & $940-2291(1578)$ & 1885 & $1546-3548(2379)$ \\
\hline
\end{tabular}




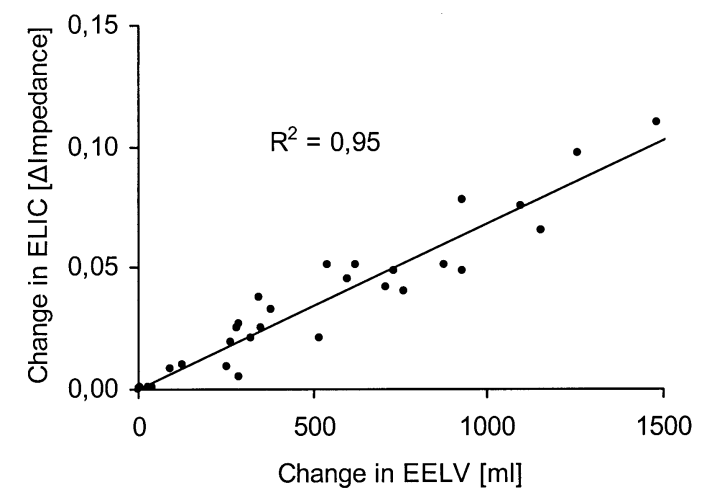

Fig. 4 Changes of the end-expiratory lung impedance change (end-expiratory lung impedance change: $y$-axis) measured by electrical impedance tomography versus changes of end-expiratory lung volume (EELV: $x$-axis) measured by multibreath nitrogenwashout manoeuvre during mechanical ventilation in ten patients. Changes of end-expiratory lung impedance change and EELV were calculated against end-expiratory lung impedance change and EELV values at PEEP $0 \mathrm{cmH}_{2} \mathrm{O}$

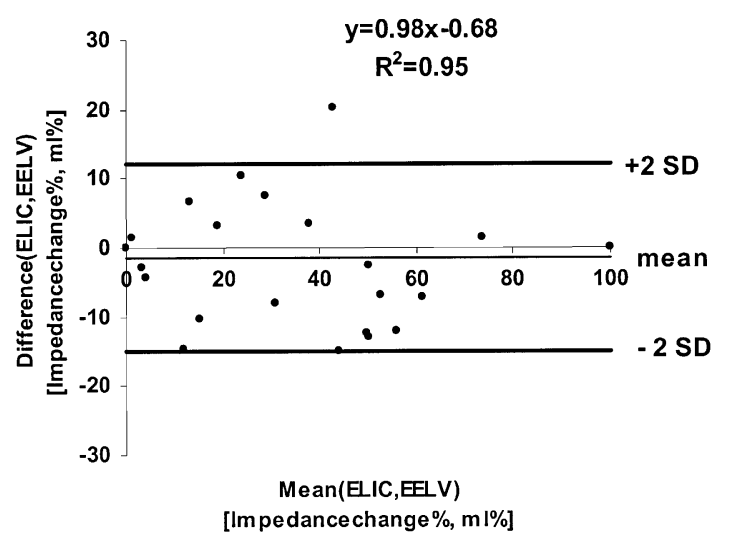

Fig. 5 Bland-Altman analysis. Comparison of end-expiratory lung impedance change (end-expiratory lung impedance change) measured with electrical impedance tomography and end-expiratory lung volume (EELV) measured by multiple breath nitrogen washout normalized to values at PEEP $15 \mathrm{cmH}_{2} \mathrm{O}$ in ten patients. The linear correlation is shown in Fig. 4. Since data were normalized relative to PEEP 0 and PEEP 15 , the bias (mean) will invariably be close to zero. Thus, a bias analysis is not meaningful. However, the random error $( \pm 2 \mathrm{SD})$ is not affected by the normalization process
The back-projection used generates relative change of impedance compared to a reference dataset at PEEP 0 . Therefore, after normalizing the change of EELV and end-expiratory lung impedance change to data at PEEP 15, a linear correlation and a Bland-Altman analysis was performed (Fig. 5). When all comparisons from the ten patients studied were pooled, a highly significant linear correlation between end-expiratory lung impedance change measured by EIT and EELV measured by multibreath nitrogen-washout manoeuvre was found, according to the equation $\mathrm{y}=0.98 \times-0.68, r^{2}=0.95$.

\section{Discussion}

This study was performed to compare end-expiratory lung volume measured by multibreath nitrogen-washout (multibreath nitrogen-washout manoeuvre) with the endexpiratory lung impedance change as measured by EIT. Multibreath nitrogen-washout manoeuvre has been thoroughly validated to measure EELV at the bedside in ICU patients [8].

EIT is a new technique, which in previous studies under laboratory conditions has been shown to identify lung volume changes $[14,15,16]$ with the potential of non-invasive monitoring lung volume changes in mechanically ventilated patients. The experimental studies have demonstrated the ability of EIT to identify incremental lung volume changes.

Our results show that a PEEP-induced increase of EELV is accompanied by a proportional increase of endexpiratory lung impedance change, but that end-expiratory lung impedance change slightly underestimates lung volume changes.

Several authors proposed the monitoring of end-expiratory lung volume as a tool to detect alveolar recruitment during mechanical ventilation $[1,3,17]$.

\section{Methodological aspects}

The basic principle of EIT is based on the measurement of relative changes of impedance compared to a refer-
Table 3 Change in end-expiratory lung impedance change (end-expiratory lung impedance change) at different PEEP-levels against reference at PEEP $0 \mathrm{cmH}_{2} \mathrm{O}$ in ten mechanically ventilated patients. Data as range and median

\begin{tabular}{lllll}
\hline Patient & PEEP 0 (Impedance) & PEEP 5 (Impedance) & PEEP 10 (Impedance) & PEEP 15 (Impedance) \\
\hline 1 & 0.000 & 0.005 & 0.040 & 0.110 \\
2 & 0.000 & 0.008 & 0.021 & 0.048 \\
3 & 0.000 & 0.010 & 0.042 & 0.097 \\
4 & 0.000 & 0.033 & 0.075 & 0.101 \\
5 & 0.000 & 0.010 & 0.020 & 0.049 \\
6 & 0.000 & 0.038 & 0.065 & 0.120 \\
7 & 0.000 & 0.001 & 0.025 & 0.051 \\
8 & 0.000 & 0.001 & 0.027 & 0.051 \\
9 & 0.000 & 0.001 & 0.025 & 0.051 \\
10 & 0.000 & 0.021 & 0.045 & 0.078 \\
& $0.000-0.000(0.000)$ & $0.001-0.038(0.009)$ & $0.020-0.075(0.034)$ & $0.048-0.130(0.065)$ \\
\hline
\end{tabular}


ence dataset within an observed object. Impedance changes within the lung are generated either by variations of the air content or changes of the central blood volume due to the pulsatile blood flow [12]. Thus, pulsatile changes of the central blood volume may interfere with lung volume measurements and may thereby increase the random error. In addition, pulmonary air content and impedance change may not correlate linearly, if stretching of lung parenchyma itself causes impedance changes. Since aeration of the lungs as well as the distribution of ventilated tidal volumes are not distributed homogeneously within the lungs, this will surely cause regional differences of lung parenchyma stretch. Furthermore, Holder and Khan [18] determined the linear operating range of impedance changes using different polyacrylamide gels and a saline-filled tank as a model together with the MarkIsystem (APT System MK1, IBEES, Sheffield, UK) for EIT measurements. They found that the measured impedance was linearly correlated to the impedance of the polyacrylamide gel, up to an increase of $20 \%$ of the reference. If gels with a higher impedance were used, the changes were underestimated by the MarkIsystem. This result may either depend on the electrical properties of the MarkI system [19] or on the image reconstruction algorithm [20] which is identical to the one we used in this study. The image reconstruction algorithm, is based on the following assumptions: the measured object has to be two-dimensional and circular, its resistivity distribution should initially be uniform, and changes in resistivity should be rather small.
Finally, the electrodes have to be located equidistant around the object. The applied respiratory settings used in our study caused an end-expiratory lung impedance change of up to 0.12 , which is $12 \%$. Although all these assumptions are in fact violated during EIT measurements it has repeatedly been shown that meaningful results can be obtained with this method $[21,22,23,24,6$, $25,26]$.

End-expiratory lung impedance change reflects impedance variations in one cross-section of the thorax, while EELV is a global parameter of the whole lung. Therefore, regional heterogeneity of the pulmonary compliance may cause slightly different results depending on the transverse slice monitored with EIT (e.g., apical versus basal region).

Nevertheless, the increase of EELV induced by stepwise increases of PEEP was proportional to end-expiratory lung impedance change, so that end-expiratory lung impedance change seems to reflect regional volume changes. In the future, the development of three-dimensional EIT [27] may provide helpful information not only about the regional volume redistribution but also about aeration and ventilation of the whole lung.

In conclusion, this study shows that EELV changes induced by PEEP during mechanical ventilation can be identified at the bedside and monitored continuously by end-expiratory lung impedance change using EIT. Thus, the method described here has the potential to be used as a simple bedside technique for the measurement of pulmonary aeration and ventilation distribution.

\section{References}

1. Hedenstierna G (1993) The recording of FRC - is it of importance and can it be made simple? Intensive care Med 19:365-366

2. Mancebo J (1992) PEEP, ARDS, and alveolar recruitment. Intensive care Med 18:383-385

3. Gattinoni L, Pelosi P, Crotti S, Valenza F (1995) Effects of positive end-expiratory pressure on regional distribution of tidal volume and recruitment in adult respiratory distress syndrome. Am J Respir Crit Care Med 151:18071814

4. Darling RC, Richards D W, Cournant A (1940) Studies on intrapulmonary mixture of gases. Open curcuit method for measuring residual air. J Clin Invest 19:609-618

5. Barber DC, Brown BH (1984) Applied potential tomography. J Phys E Sci Instrum 17:723-733
6. Frerichs I (2000) Electrical impedance tomography (EIT) in applications related to lung and ventilation: a review of experimental and clinical activities. Physiol Meas 21:R1-21

7. Murray JF, Matthay MA, Luce JM, Flick MR (1988) An expanded definition of the adult respiratory distress syndrome [published erratum appears in Am Rev Respir Dis 1989 139:1065] Am Rev Respir Dis 138:720-723

8. Wrigge H, Sydow M, Zinserling J, Neumann P, Hinz J, Burchardi H (1998) Determination of functional residual capacity (FRC) by multibreath nitrogen-washout in a lung model and in mechanically ventilated patients. Accuracy depends on continuous dynamic compensation for changes of gas sampling delay time. Intensive Care Med 24:487-493

9. Brunner JX, Langenstein H, Wolff G (1983) Direct accurate gas flow measurement in the patient: compensation for unavoidable error. Med Prog Technol 9:233-238
10. Cournand A, Yarmouth IG, Riley RL (1941) Influence of body size on gaseous nitrogen elemination during high oxygen breathing. Proc Soc Exp Biol 48:280-284

11. Barber DC, Brown BH (1986) Recent developments in applied potential tomography-APT. In: Bacharach SL (ed) Proc 9th Conference of Information Processing in Medical Imaging. Martinus Nijhoff, Dordrecht, pp 106-121

12. Faes TJ, van der Meij HA, de Munck JC, Heethaar RM (1999) The electric resistivity of human tissues $(100 \mathrm{~Hz}-$ $10 \mathrm{MHz}$ ): a meta- analysis of review studies. Physiol Meas 20:R1-10

13. Bland JM, Altman DG (1986) Statistical methods for assessing agreement between two methods of clinical measurement. Lancet 307-310

14. Hahn G, Sipinkova I, Baisch F, Hellige $G$ (1995) Changes in the thoracic impedance distribution under different ventilatory conditions. Physiol Meas 16:A161-A173 
15. Adler A, Amyot R, Guardo R, Bates JH, Berthiaume Y (1997) Monitoring changes in lung air and liquid volumes with electrical impedance tomography. J Appl Physiol 83:1762-1767

16. Adler A, Shinozuka N, Berthiaume Y, Guardo R, Bates JH (1998) Electrical impedance tomography can monitor dynamic hyperinflation in dogs. J Appl Physiol 84:726-732

17. Dambrosio M, Roupie E, Mollet JJ, Anglade M C, Vasile N, Lemaire F, Brochard L (1997) Effects of positive end-expiratory pressure and different tidal volumes on alveolar recruitment and hyperinflation. Anesthesiology 87:495-503

18. Holder DS, Khan A (1994) Use of polyacrylamide gels in a saline-filled tank to determine the linearity of the Sheffield Mark 1 electrical impedance tomography (EIT) system in measuring impedance disturbances. Physiol Meas 15 [Suppl 2a]:A45-A50
19. Brown BH, Seagar AD (1987) The Sheffield data collection system. Clin Phys Physiol Meas 8:91-97

20. Barber DC (1990) Quantification in impedance imaging. Clin Phys Physiol Meas 11 Suppl A:45-56

21. Kunst PW, de Vries PM, Postmus PE, Bakker J (1999) Evaluation of electrical impedance tomography in the measurement of PEEP-induced changes in lung volume. Chest 115:1102-1106

22. Kunst PW, Vazquez DA, Bohm SH, Faes TJ, Lachmann B, Postmus PE, de Vries PM (2000) Monitoring of recruitment and derecruitment by electrical impedance tomography in a model of acute lung injury. Crit Care Med 28:3891-3895

23. Kunst PW, Vonk Noordegraaf AV, Raaijmakers E, Bakker J, Groeneveld AB, Postmus PE, de Vries P M (1999) Electrical impedance tomography in the assessment of extravascular lung water in noncardiogenic acute respiratory failure. Chest 116:1695-1702
24. Vonk NA, Kunst PW, Janse A, Smulders RA, Heethaar RM, Postmus PE, Faes T J, de Vries PM (1997) Validity and reproducibility of electrical impedance tomography for measurement of calf blood flow in healthy subjects. Med Biol Eng Comput 35:107-112

25. Frerichs I, Hahn G, Golisch W, Kurpitz M, Burchardi H, Hellige G (1998) Monitoring perioperative changes in distribution of pulmonary ventilation by functional electrical impedance tomography. Acta Anaesthesiol Scand 42:721-726

26. Frerichs I, Hahn G, Schiffmann H, Berger C, Hellige G (1999) Monitoring regional lung ventilation by functional electrical impedance tomography during assisted ventilation. Ann NY Acad Sci 873:493-505

27. Metherall P, Barber DC, Smallwood RH, Brown BH (1996) Threedimensional electrical impedance tomography. Nature 380:509-512 


\title{
Effect of positive end-expiratory-pressure on regional ventilation in patients with acute lung injury evaluated by electrical impedance tomography
}

\author{
J. Hinz, O. Moerer, P. Neumann, T. Dudykevych, G. Hellige, M. Quintel \\ University Göttingen, Emergency and Intensive Care Medicine, Department of Anaesthesiology, Göttingen, Germany
}

\begin{abstract}
Summary
Background and objective: For the treatment of patients with adult respiratory distress syndrome and acute lung injury bedside measurements of regional lung ventilation should be considered for optimizing ventilatory settings. The aim was to investigate the effect of positive end-expiratory pressure (PEEP) on regional ventilation in mechanically ventilated patients at the bedside by electrical impedance tomography. Methods: Eight mechanically ventilated patients were included in the study. PEEP levels were increased from 0 to 5, 10, 15 mbar and back to 0 mbar. Regional ventilation in 912 regions of the thorax was investigated at each PEEP by electrical impedance tomography. The obtained regions were divided in four groups: none (none and poorly ventilated regions including chest wall and mediastinum), bad, moderate and well-ventilated regions. Results: Increasing the PEEP stepwise from 0 to 15 mbar decreased the non-ventilated regions (none: 540 regions at PEEP 0 and 406 regions at PEEP 15). In contrast, the other regions increased (bad: 316 regions at PEEP 0 and 380 regions at PEEP 15; moderate: 40 regions at PEEP 0 and 100 regions at PEEP 15; well: 0 region at PEEP 0 and 34 regions at PEEP 15 (median values)) indicating an improvement of regional ventilation. Conclusions: Increasing PEEP in mechanically ventilated patients reduces none ventilated regions (atelectasis). Furthermore, it leads to a shift from none and bad ventilated regions to moderately and well-ventilated regions. Electrical impedance tomography is a bedside technique and might be an alternative to computed tomography scan to assess aerated lung regions.
\end{abstract}

Keywords: THORACIC ELECTRICAL IMPEDANCE TOMOGRAPHY, regional ventilation, positive end-expiratory pressure.

\section{Introduction}

Mechanical ventilation combined with positive endexpiratory pressure (PEEP) was first described in patients suffering from lung oedema caused by cardiac dysfunction [1]. Since its introduction into clinical practice by Ashbaugh and colleagues [2], this ventilatory strategy is well accepted as part of the standard therapy for patients with acute respiratory failure

Correspondence to: José Hinz, Department of Anaesthesiology, Emergency and Intensive Care Medicine, Georg-August-University, Robert-Koch-Strasse 40, D-37075 Göttingen, Germany. E-mail: mail@josehinz.de; Tel: + 49551 398826; Fax: +49551398676

Accepted for publication August 2005 EJA 3251 requiring mechanical ventilation [3]. Several authors showed that PEEP improves oxygenation by increasing lung volume [4-7]. This is caused by recruitment of non-ventilated lung regions, so-called atelectasis $[8,9]$ or by stretching (hyperinflating) already opened alveoli [10]. Today it is assumed, that an inappropriate PEEP may induce secondary lung injury [11]. An increase of airway pressure above lung compliance causes micro-injuries of the alveolar membranes. In case of higher airway pressure alveolar rupture may occur, which leads to pneumothorax or pneumomediastinum.

Gattinoni showed an inhomogeneous distribution of tidal volume and recruitment in adult respiratory 
distress syndrome (ARDS) [12]. And recently, differences in regional compliances were measured in a large series of ARDS patients [13]. From this, an optimal PEEP should be based on a compromise between recruitment and lung over distension [14]. For the treatment of patients with ARDS and acute lung injury (ALI) regional inhomogeneities of the diseased lung should be considered for optimizing ventilatory settings. In principle regional aeration can be obtained by computed tomography (CT). However, a CTscanner is not available at the bedside and is associated with radiation load. Electrical impedance tomography (EIT) measures radiation-free at the bedside regional aeration and ventilation $[15,16]$. Recently, a number of investigators have focused on EIT applications in pulmonary imaging. Adler and colleagues $[17,18]$ used an EIT system to generate dynamic images of impedance change in an uninjured animal lung. EIT described accurately lung volume changes during incremental increases of gas volume in conventional mechanical ventilation. Kunst and colleagues [19] used an EIT device to describe regional impedance changes in a saline lavaged lung injury model. Impedance changes correlated closely with whole lung pressure-volume relationships quantified by strain gauge plethysmography. The author observed marked differences in the pressure volume curves between the anterior and posterior part of the lung. The same group of investigators described the anterior-posterior lung impedance changes during recruitment and derecruitment in an animal model [20]. This study shows the potential of EIT to provide dynamic, non-invasive information about differences in regional lung ventilation. The aim of the study was to investigate the influence of increasing PEEP on regional ventilation in mechanically ventilated patients at the bedside by EIT.

\section{Patients and methods}

\section{Study protocol}

After approval of the local Ethics Committee the study was performed in the intensive care unit of the
Department of Anaesthesiology, Emergency and Intensive Care Medicine, University Hospital Göttingen, Germany. An informed written consent to participate in this study was obtained from the closest relatives of eight mechanically ventilated patients. For patient characteristics see Table 1 .

Inclusion criteria were:

- Mechanically ventilated patient $\geqslant 24 \mathrm{~h}$ before onset of the study

- ALI $\left(\mathrm{PaO}_{2} / \mathrm{FiO}_{2}<300 \mathrm{mmHg}\right)$

- Age $\geqslant 18$ yr

- Clinically indicated arterial catheter.

Exclusion criteria were:

- Pregnancy

- Terminal illness

- Unstable haemodynamics

- Cardiac pacemakers

- Thoracic or cardiac surgery.

All patients were ventilated with a ventilator (Evita 2; Dräger AG, Lübeck, Germany) in a volumecontrolled mode with constant tidal volume and respiratory rate. Tidal volume, inspiratory oxygen $\left(\mathrm{FiO}_{2}\right)$ concentration and PEEP were set by the attending physician in order to achieve normocapnia $\left(\mathrm{PaCO}_{2}\right.$ $35-45 \mathrm{mmHg})$ and oxyhaemoglobin saturation $\left(\mathrm{SaO}_{2}\right)$ $>95 \%$. At the beginning of the study PEEP was decreased to 0 mbar and occasionally the $\mathrm{FiO}_{2}$ was increased so that the $\mathrm{SaO}_{2}$ remained above $90 \%$. Thereafter, PEEP was increased stepwise to 5, 10 and 15 mbar approximately after intervals of $40 \mathrm{~min}$ when steady-state condition had been achieved. At the end of each study period end-expiratory lung volume (EELV) was measured by an open circuit multibreath nitrogen washout manoeuvre (MBNW). Simultaneously, measurement of regional ventilation was performed by EIT. Additionally, we investigated oxygen saturation, carbon dioxide and arterial oxygen pressure by arterial blood gas samples (ABL 300 and OSM 3; Hemoximeter Radiometer, Copenhagen, Denmark).

Table 1. Patient characteristics.

\begin{tabular}{|c|c|c|c|c|c|c|}
\hline Patient & Age (yr) & Height $(\mathrm{cm})$ & Weight (kg) & Diagnosis & Lung diagnosis & Lung injury score \\
\hline 1 & 37 & 178 & 80 & Skull brain injury & Atelectasis & 1.0 \\
\hline 2 & 78 & 165 & 65 & Hemicolektomie & Atelectasis & 1.0 \\
\hline 3 & 30 & 182 & 80 & Skull brain injury & Atelectasis & 1.3 \\
\hline 4 & 78 & 167 & 60 & & Pneumonia & 3.0 \\
\hline 5 & 62 & 180 & 90 & Subarachnoid haemorrhage & ARDS & 3.3 \\
\hline 6 & 58 & 168 & 65 & Lung cancer & Pneumonia & 1.0 \\
\hline 7 & 33 & 170 & 80 & Polytrauma & ARDS & 2.0 \\
\hline 8 & 69 & 185 & 115 & & ARDS & 2.3 \\
\hline Range (median) & $37-78(60)$ & $165-185(170)$ & $60-115(80)$ & & & $1.0-3.3(1.3)$ \\
\hline
\end{tabular}




\section{End-expiratory lung volume}

EELV was calculated by an modified open circuit MBNW [21,22]. Briefly, gas flow was measured with a pneumotachograph (Fleisch No. 2; Fleisch, Lausanne, Switzerland) and a differential pressure transducer (Huba Control, Würenlos, Switzerland) directly connected to a heat and moisture exchanger (HumidVent 2; Gibeck Respiration, Väsby, Sweden) at the endotracheal tube. Inspiratory and expiratory gas was continuously sampled via a capillary (length: $3.09 \mathrm{~m}$, flow: $1 \mathrm{~mL} \mathrm{~s}^{-1}$ ) connected to the y-piece of the breathing circuit. Nitrogen $\left(\mathrm{N}_{2}\right)$, oxygen $\left(\mathrm{O}_{2}\right)$ and carbon dioxide $\left(\mathrm{CO}_{2}\right)$ were measured by a mass spectrometer (MGA 1100; Perkin Elmer, Pomona, CA, USA). All data were sampled online by an analogue to digital converter (DT2801-A; Data Translation, Marlboro, MA, USA) at a rate of $40 \mathrm{~Hz}$ and processed by an IBM AT compatible personal computer. The data acquisition and processing software was programmed with a commercially available software program (Asyst ${ }^{\circledR}$ 4.0; Keithley Asyst, Taunton, MA, USA). Calculation of EELV was performed offline.

\section{Electrical impedance tomography}

Regional ventilation was studied by EIT [15] (APT System MK1, IBEES, Sheffield, UK). Briefly, 16 surface electrodes (Blue Sensor VL-50-K; Medicotest, Olstykke, Denmark) were placed equidistantly on the circumference of the thorax in a transversal plane at sixth intercostal space parasternal and one reference electrode at the abdomen (Fig. 1). For data collection an alternate current $(5 \mathrm{~mA}$ p by $\mathrm{p}, 50 \mathrm{kHz})$ was injected between one pair of adjacent electrodes. The resulting surface potentials depend on the impedance inside the thorax and are measured between the remaining adjacent electrode pairs. All 16 electrode pairs were used, one pair after the other, as injecting electrodes. One EIT image was completed when all pairs of adjacent electrodes had been used once as injecting electrodes.

According to the method described by Barber and Brown the resulting 208 surface potentials were normalized to the mean surface potential (reference) during the measurement period. These normalized surface potentials were subsequently used for the reconstruction of regional impedance changes by a modified back-projection [23]. This modified back-projection algorithm delivers regional impedance changes with respect to the reference in 912 regions-of-interest within a circular area in a $32 \times 32$ pixel matrix. Inspiration leads to an increase in regional impedance, while expiration leads to a decrease in regional impedance [24]. Repeated measurements of 1000 EIT images with a sampling rate of 10 EIT images per second resulted in a study period of $100 \mathrm{~s}$, in which regional ventilation was calculated.

In this investigation commercially available electrodes were used for EIT measurements. Hairy patients were shaved at the sites where the electrodes were applied to ensure optimal contact. The used software for data acquisition with the EIT system allows measuring continuously the quality of the contact between skin and electrodes. Thereby inadequate electrode contact can be detected.

Calculation of regional ventilation. Functional electrical impedance tomography (f-EIT) images were calculated to determine regional lung ventilation [25]. The f-EIT image is calculated from 1000 EIT images and shows the regional impedance amplitude of $32 \times 32$ regions between end inspiration and end expiration. Examples of an f-EIT image at different PEEP are shown in Figure 2. For further evaluation the regions of this f-EIT image were distributed with reference to the highest regional amplitude into four groups:

- None: regions were impedance amplitude was within $0-10 \%$ of the reference.

- Bad: regions were impedance amplitude was within $10-30 \%$ of the reference.

- Moderate: regions were impedance amplitude was within 30-70\% of the reference.

- Well: regions were impedance amplitude was within $70-100 \%$ of the reference.

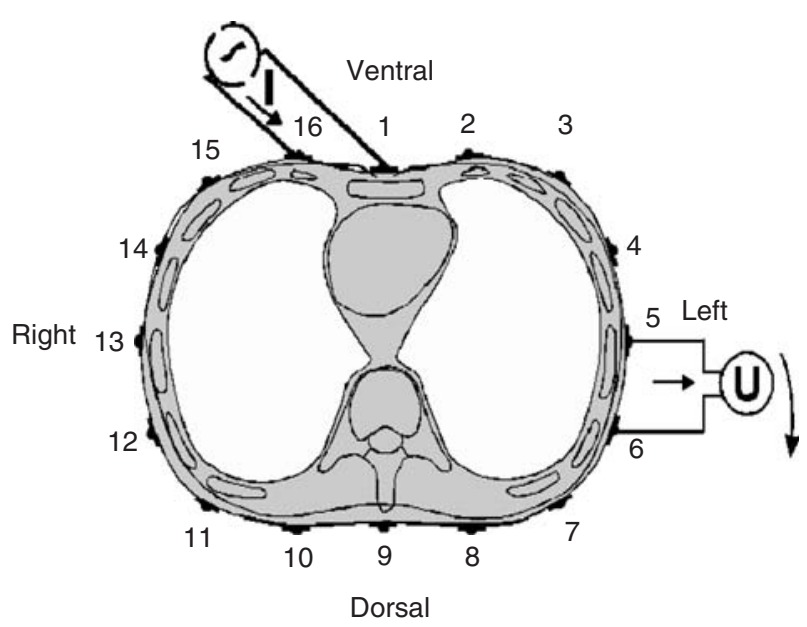

Figure 1.

Principle of EIT. For data collection an alternating current $(5 \mathrm{~mA}$ p by $p, 50 \mathrm{kHz}$ ) was injected between one pair of adjacent electrodes. The resulting potential differences were measured between the remaining adjacent electrode pairs. All 16 adjacent electrode pairs were used, one pair after the other, as injecting electrodes with the potential differences measured with the remaining electrodes. One EIT image was completed when all pairs of adjacent electrodes had been used once as injecting electrodes. 


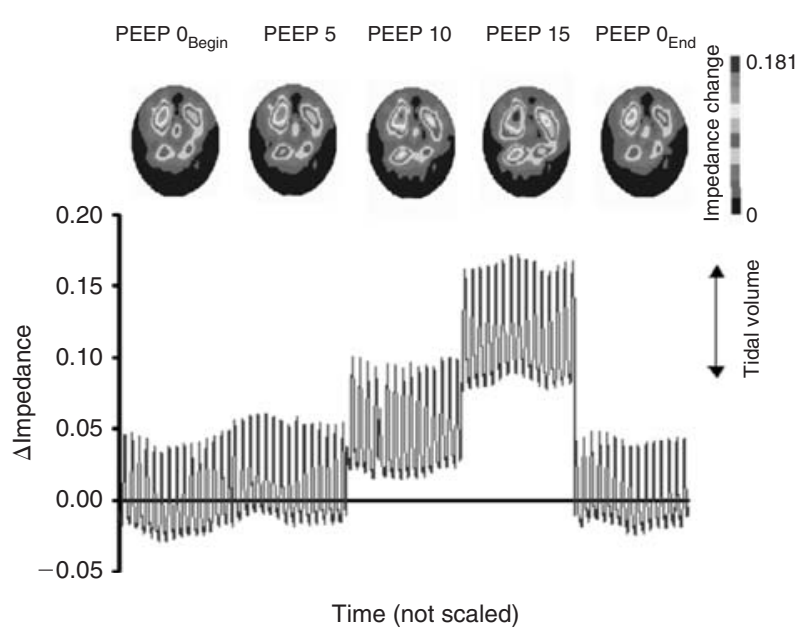

Figure 2.

Five example of $f$-EIT image and lung impedance change (impedance) in a transversal plane at sixth intercostal space in a mechanically ventilated patient at different PEEP. The generation of $f$-EIT is based on the assessment of the amplitude of impedance changes in every region from series of EIT images. Inspiration leads to an increase in impedance, while expiration leads to a decrease in impedance. Therefore, an f-EIT image shows the regional amplitude of impedance change during end inspiration and end expiration.

\section{Statistics}

Calculations were performed using the STATISTICA software package (Statistica 5.1; StatSoft Inc, Tulsa, OK, USA) on a personal computer (Pentium III $800 \mathrm{MHz}$; Microsoft Windows XP). Since the data were not normally distributed, as tested by the Kolmogorov-Smirnov test, all data are presented as median and range (min-max (median)) unless stated otherwise. We subsequently analyse differences at different PEEP by Wilcoxon signed rank sum test. For all statistical tests $P<0.05$ was considered to be significant.

\section{Results}

\section{Gas exchange and EELV}

Arterial carbon dioxide pressure remained stable during all PEEPs $(40-42 \mathrm{mmHg}$ ). The arterial oxygen saturations were $99-100 \%$ and remained also stable during all PEEPs. Oxygenation calculated by the arterial $\mathrm{PaO}_{2} / \mathrm{FiO}_{2}$ ratio (Horovitz index) improved significantly during increase of PEEP. Oxygenation varied from $215 \mathrm{mmHg}$ at PEEP 0 to $286 \mathrm{mmHg}$ at PEEP 15, which was an increase of $33 \%$. After return to PEEP 0, oxygenation remained significantly higher $(240 \mathrm{mmHg})$ than the initial oxygenation at PEEP $0(215 \mathrm{mmHg})$ (Fig. 3).

EELV improved significantly during increasing PEEP. EELV ranged from $1316 \mathrm{~mL}$ at PEEP 0 to $2561 \mathrm{~mL}$ at PEEP 15, which was an enlargement of

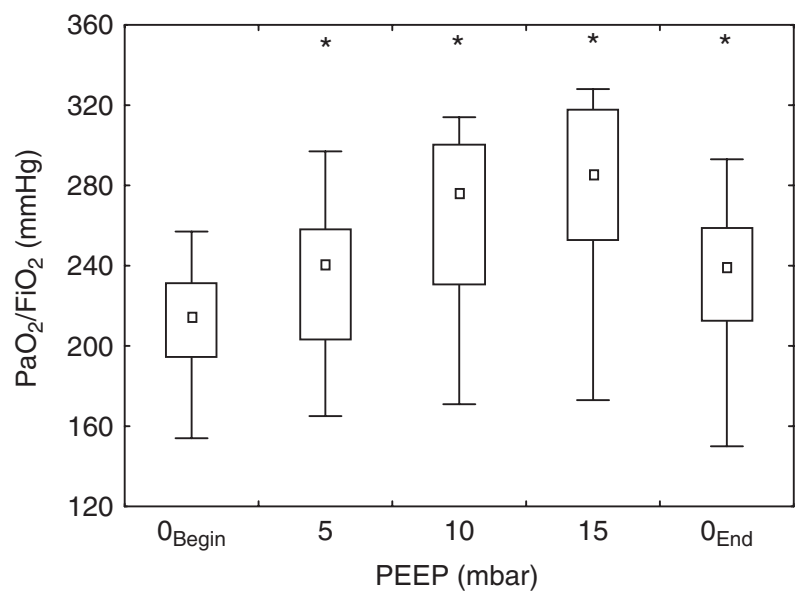

Figure 3.

$\mathrm{PaO}_{2} / \mathrm{FiO}_{2}$ at different PEEP in eight mechanically ventilated patients (range and median). ${ }^{*} \mathrm{P}<0.05$ against reference PEEP $O_{\text {Begin }}$.

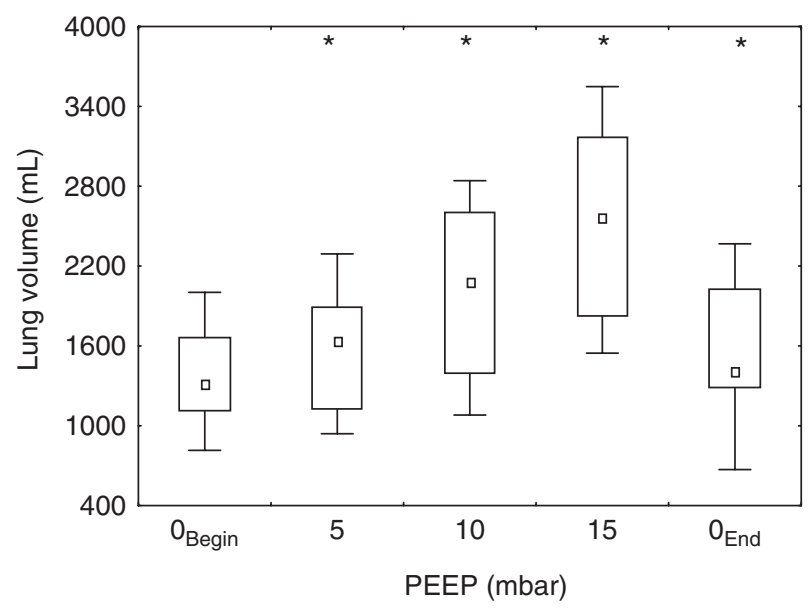

Figure 4 .

Box-plots of EELV at different PEEP in eight mechanically ventilated patients (range and median). ${ }^{*} \mathrm{P}<0.05$ against reference at PEEP $O_{\text {Begin }}$.

95\%. We found an EELV after return to PEEP 0 $(1409 \mathrm{~mL})$, which was significantly higher compared to the initial PEEP 0. All data are summarized in Figure 4.

\section{Regional ventilation}

The 'none' ventilated group contains atelectasis, chest wall and mediastinum. During increased PEEP the number of regions decreased by $25 \%$ significantly from 540 regions at PEEP 0 down to 406 regions at PEEP 15. Returning to PEEP 0 resulted in 455 regions not ventilated, which were 85 regions less than at the initial PEEP 0. The 'bad' ventilated group increased also significantly by $20 \%$ from 316 regions at PEEP 0 to 380 regions at PEEP 15 . The 'moderate' group 
Table 2. Counts of regions, where different ventilation was found at different PEEP from eight mechanically ventilated patients. By increasing PEEP we found an increase of ventilated regions, caused by redistribution from none ventilated regions to bad, moderate and well-ventilated regions.

\begin{tabular}{|c|c|c|c|c|c|c|}
\hline Patient & & PEEP $0_{\text {Begin }}$ & PEEP 5 & PEEP 10 & PEEP 15 & PEEP $0_{\text {End }}$ \\
\hline \multirow[t]{4}{*}{1} & None & 507 & 494 & 448 & 362 & 504 \\
\hline & Bad & 381 & 393 & 409 & 424 & 390 \\
\hline & Moderate & 24 & 25 & 53 & 100 & 18 \\
\hline & Well & 0 & 0 & 2 & 26 & 0 \\
\hline \multirow[t]{4}{*}{2} & None & 613 & 566 & 521 & 475 & 421 \\
\hline & $\mathrm{Bad}$ & 268 & 303 & 335 & 351 & 435 \\
\hline & Moderate & 31 & 43 & 55 & 66 & 56 \\
\hline & Well & 0 & 0 & 1 & 20 & 0 \\
\hline \multirow[t]{4}{*}{3} & None & 608 & 551 & 418 & 378 & 513 \\
\hline & $\mathrm{Bad}$ & 299 & 341 & 402 & 394 & 374 \\
\hline & Moderate & 5 & 20 & 82 & 108 & 25 \\
\hline & Well & 0 & 0 & 10 & 32 & 0 \\
\hline \multirow[t]{4}{*}{4} & None & 478 & 417 & 341 & 250 & 407 \\
\hline & $\mathrm{Bad}$ & 353 & 412 & 472 & 492 & 416 \\
\hline & Moderate & 78 & 79 & 90 & 135 & 75 \\
\hline & Well & 3 & 4 & 9 & 35 & 14 \\
\hline \multirow[t]{4}{*}{5} & None & 556 & 527 & 478 & 408 & 474 \\
\hline & Bad & 313 & 337 & 369 & 390 & 383 \\
\hline & Moderate & 43 & 45 & 47 & 72 & 46 \\
\hline & Well & 0 & 3 & 18 & 42 & 9 \\
\hline \multirow[t]{4}{*}{6} & None & 584 & 537 & 454 & 404 & 531 \\
\hline & Bad & 290 & 333 & 378 & 369 & 340 \\
\hline & Moderate & 36 & 36 & 61 & 99 & 35 \\
\hline & Well & 2 & 6 & 19 & 40 & 6 \\
\hline \multirow[t]{4}{*}{7} & None & 523 & 501 & 462 & 445 & 424 \\
\hline & Bad & 343 & 346 & 352 & 334 & 412 \\
\hline & Moderate & 46 & 65 & 88 & 116 & 75 \\
\hline & Well & 0 & 0 & 10 & 17 & 1 \\
\hline \multirow[t]{4}{*}{8} & None & 514 & 489 & 453 & 420 & 435 \\
\hline & $\mathrm{Bad}$ & 318 & 358 & 375 & 365 & 384 \\
\hline & Moderate & 62 & 44 & 55 & 90 & 68 \\
\hline & Well & 18 & 21 & 29 & 37 & 25 \\
\hline \multirow[t]{4}{*}{ Overall } & None & $478-613(540)$ & $417-566(514)$ & $341-521(454)$ & $250-475(406)$ & $407-531(455)$ \\
\hline & $\mathrm{Bad}$ & $268-381(316)$ & $303-412(343)$ & $335-472(377)$ & $334-492(380)$ & $340-435(387)$ \\
\hline & Moderate & $5-78(40)$ & $20-79(44)$ & $47-90(58)$ & $66-135(100)$ & $18-75(100)$ \\
\hline & Well & $0-18(0)$ & $0-21(1)$ & $1-29(10)$ & $17-42(34)$ & $0-25(4)$ \\
\hline
\end{tabular}

increased significantly 2.5 -fold from 40 regions at PEEP 0 to 100 regions at PEEP 15 . And, the 'well' group increased significantly from 0 region at PEEP 0 to 34 regions at PEEP 15 . All data are summarized in Table 2, Figures 5 and 6.

\section{Discussion}

The aim of the study was to investigate the effect of increasing PEEP levels on regional ventilation in mechanically ventilated patients at bedside with EIT. We found an improvement in oxygenation during stepwise increase of PEEP from 0 to 5, 10, 15 mbar. This improvement in oxygenation was accompanied by an increase of EELV. This increase of EELV was caused by a decrease of non-ventilated lung regions. Non-ventilated regions were recruited toward bad, moderate and well-ventilated regions.
Electrical impedance tomography. EIT is an imaging technique offering the possibility of regional lung function studies [26]. It was developed in the early 1980s by Barber and Brown and bases on an alternate current injection and voltage measurement via 16 surface electrodes placed around the thorax [15]. All 16 adjacent electrode pairs are used, one pair after the other, as injecting electrodes while surface potentials are measured with the remaining 13 electrode pairs. One data collection cycle is completed when all pairs of adjacent electrodes have been used once as injecting electrodes. The sampling rate of the used EIT system is 10 cycles s $^{-1}$. The resulting 208 surface potentials ( 16 current injections $\times 13$ surface potentials) per cycle are normalized to the mean surface potential. These normalized potentials are used by a back-projection for reconstruction of regional impedance changes following changes of aeration in 912 

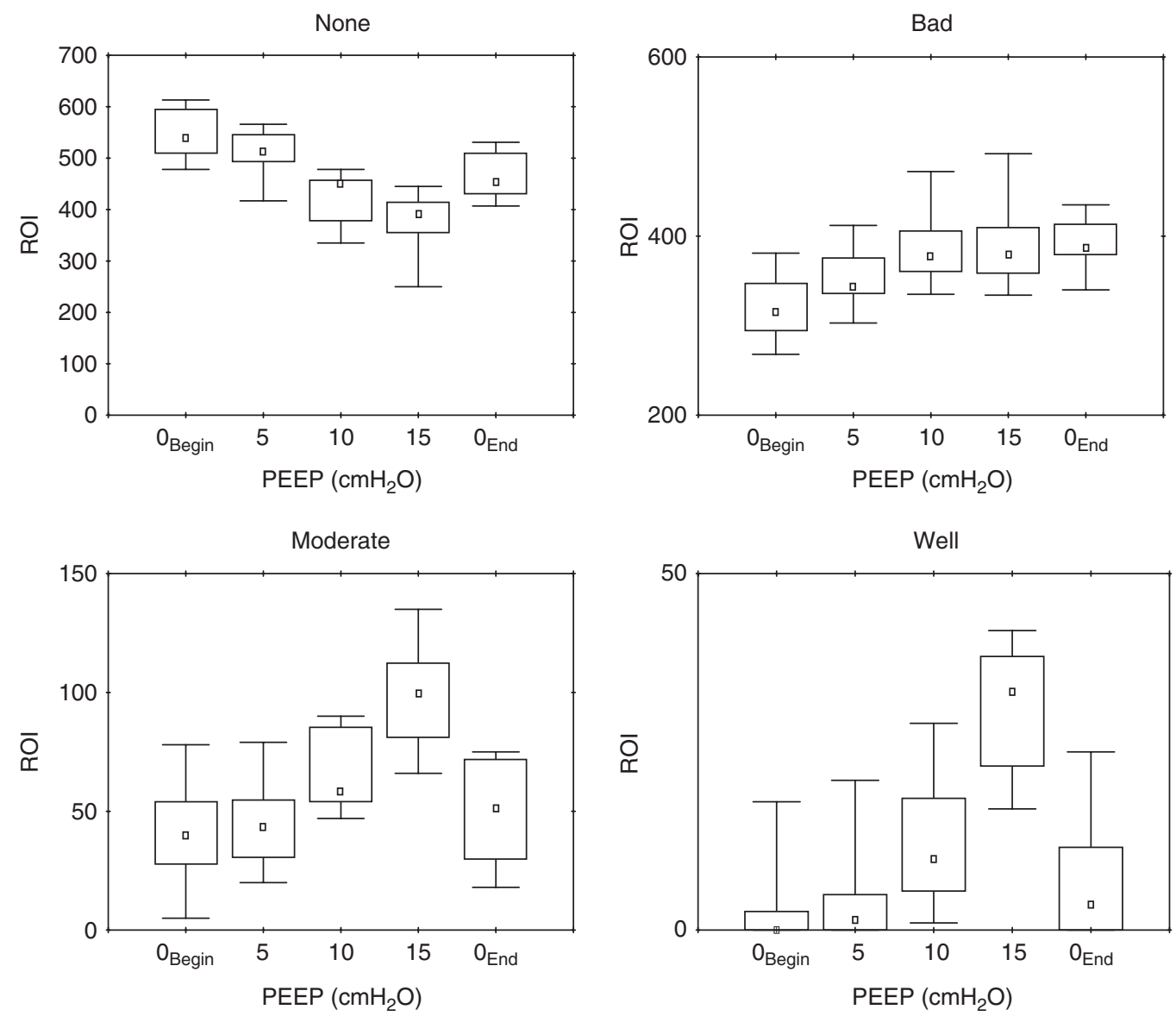

Figure 5 .

Counts of regions (ROI), where different ventilation was found at different PEEP-levels summarized from eight mechanically ventilated patients. During increased PEEP the number of none ventilated regions decreased significantly. The other ventilated regions increased significantly from PEEP 0 to PEEP 15.

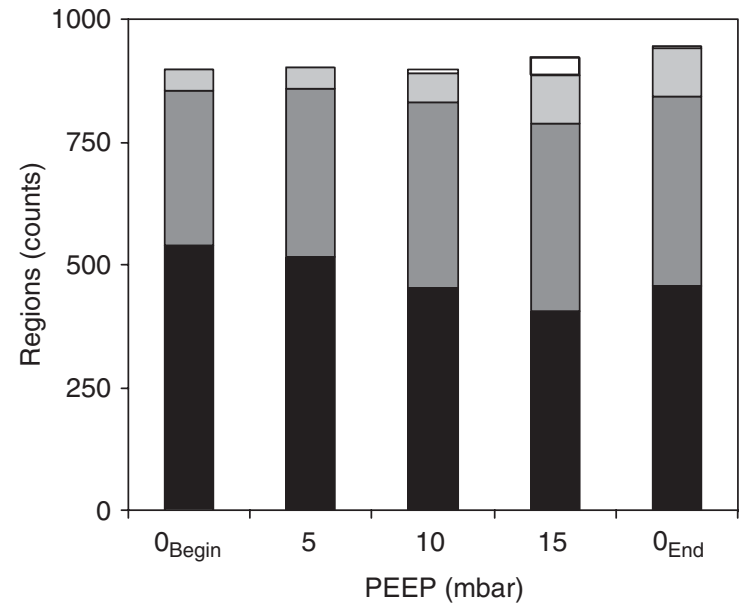

$\square$ Well $\square$ Moderately $\square$ Badly $\square$ None

Figure 6.

Distribution of ventilated regions (well, moderately, bad, none) at different PEEP-levels summarized from eight mechanically ventilated patients. Note that during increasing PEEP redistribution from none to badly, moderately and well-ventilated regions occurred. regions within a thoracic plane [25]. EIT was validated in pigs with the established techniques of $\mathrm{CT}$ and ventilation scintigraphy comparing regional ventilation during different tidal and lung volumes $[24,27]$. These studies confirmed that EIT is a suitable technique for detecting regional changes in air content (regional ventilation) and the monitoring of local effects of mechanical ventilation.

EIT is suitable for monitoring regional lung ventilation, regional tidal volume, regional lung volume and functional residual capacity non-invasively at the bedside [28-30]. In neonates, where radiation exposure is undesirable, EIT may even replace $\mathrm{X}$-ray investigations in certain situations [31]. However, EIT should not be applied to obtain morphological information similar to CT or magnetic resonance imaging, since the latter diagnostic methods provide anatomical information with a higher spatial and morphological resolution. The major advantages of EIT are that it is non-invasive, easy to use at the bedside and that data collection can be performed with a high time resolution and acceptable local resolution. 
According to Hahn and colleagues, the detectable lung volume by EIT ranges from 9 to $29 \mathrm{~mL}$ [32]. Barber and Brown found a spatial resolution of approximately $8 \%$ of the thorax diameter, so that a resolution of $8 \mathrm{~mL}$ is achieved [33]. Since its introduction the hardware and software have been improved continuously [34,35]. Hahn and colleagues developed more advanced algorithms for the analysis of dynamic physiological phenomena with low amplitudes. Thus, they introduced the f-EIT and averaging technique [25], which enables regional lung function studies.

Until now, regional lung function studies were performed by CT or ventilation scintigraphy of the lungs. However, both methods lead to radiation exposure and cannot be used at the bedside. The time resolution of both methods is rather poor. A fast speed CT is able to scan four CT images per second [36,37]. A ventilation scintigraphy [38] delivers one regional aeration image per $16 \mathrm{~min}$. Therefore, following changes in regional ventilation during fast physiological events, like PEEP change cannot be detected by these methods.

One important methodological aspect is that it is limited to a single transverse thoracic plane. It has been demonstrated, that aeration gradients are found not only in the anterior-posterior but also in the cephalo-caudal direction [13,39]. During mechanical ventilation the lungs may displace in the caudal direction, so that EIT is measuring different lung planes. This is the same criticism against CT studies, which have been shown to be misleading in the presence of cranial to caudal heterogeneity. Therefore, analysing regional ventilation in a single transversal plane may underestimate heterogeneity of regional ventilation. In future the introduction of additionally EIT planes or an optimized current injection pattern may solve this problem [40].

An EIT image summarizes impedance variation from the thorax including lung, chest wall and mediastinum induced by varying air content as well as the pulsatile bloodflow [41] within tissues [40]. In this study we assumed that impedance change above $10 \%$ the maximal impedance variation is caused by atelectasis which may lead to an underestimation [42]. In future EIT systems with an improved signal-to-noise ratio may offers the possibility of new concept for the detection of atelectasis [43]. One possible approaches may base on the fact that impedance change in the outer boundary of the EIT image is generated solely by the chest wall and not by atelectasis. Therefore, the limit of impedance change, defining lung regions will be defined for each patient individually.

Effects of PEEP on EELV. In our patients, suffering from ALI, we found an increase of EELV by $95 \%$ if PEEP was raised from 0 to $15 \mathrm{mbar}$. Gattinoni and colleagues found a comparable increase of lung volume from $18 \mathrm{~mL}$ up to $34 \mathrm{~mL}$ by rising the PEEP from 0 to $20 \mathrm{mbar}$, in mechanically ventilated patients suffering from ARDS [12]. The difference in lung recruitment (95\% our study vs. $88 \%$ Gattinoni study) besides the higher PEEP used in the Gattinoni study might caused by the severity of lung injury (ALI our study vs. ARDS Gattinoni study) and the different methods of lung imaging. Katz and colleagues found a lower increase of lung volume $(1.5-2.68 \mathrm{~L}$; increase of $79 \%$ ) in mechanically ventilated patients suffering from ALI during increase of PEEP 3 up to PEEP 18 compared to our study (1.3-2.6 L; increase of $95 \%$ ) [10]. Furthermore, they found time-dependent increase of lung volume within $1 \mathrm{~min}$ after increase of PEEP. In our study we found a similar time-dependent increase of lung volume, which occurs within 5-8 breaths after PEEP change.

Effects of PEEP on regional ventilation. By highering PEEP we found an increase of ventilated regions, caused by redistribution from none ventilated regions to bad, moderate and well-ventilated regions (Table 2, Figs 5 and 6). The sustained improvement in regional ventilation seen on return to zero PEEP may be caused by the increase of PEEP in our study protocol similar to a PEEP trial, which is used as a manoeuvre for lung recruitment [44]. In our study the 'none' ventilated regions consist of atelectasis, chest wall and mediastinum, because EIT does not discriminate between these tissues. We assumed that chest wall and mediastinum remain constant during the different PEEP levels. Therefore, changes in the number of 'none' ventilated regions are induced by a change in atelectasis.

In mechanically ventilated patients atelectasis occurs mainly in the dorsal part of the lungs. This is caused by the ventral-to-dorsal hydrostatic pressure gradient inside the lungs and the reduced movement of the dorsal part of the diaphragm [45]. PEEP prevents the collapse of a given lung region when it is equal to or greater than the hydrostatic pressure superimposed [46], this is confirmed by our data, which shows a recruitment of non-ventilated regions and an improved gas exchange during higher PEEP levels. We found a shift of the centre of ventilation to the dorsal part of the lungs during stepwise increase of PEEP (Fig. 7). An increase of ventilation in the dorsal part of the lungs and a decrease in the ventral part of the lungs by increasing PEEP was also found in other studies [12,47].

A peak airway pressure (PEEP+inspiratory pressure) above lung compliance may cause stretch of already open alveoli (hyperinflation) and possible leads to micro injuries of the alveolar membranes. Pressure trauma (barotrauma) has been attributed to the peak airway pressure. Attention has also been 


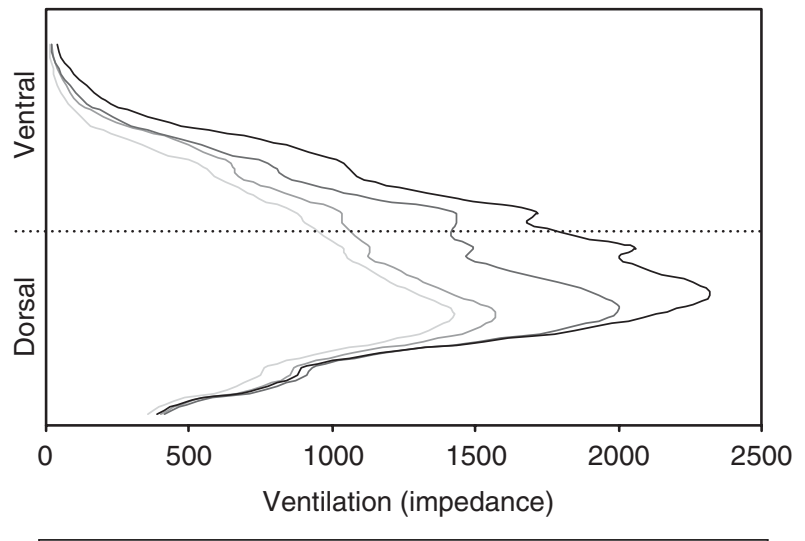

PEEP 0 - PEEP 5 - PEEP 10 - PEEP 15

Figure 7.

Means of ventilation of eight mechanically ventilated patient at different PEEP in dorsal to ventral direction in an about $4 \mathrm{~cm}$ slice of the thorax measured by EIT. Note that during increasing PEEP, improvement of regional ventilation predominate occurs in the dorsal parts of the thorax.

focused on volume trauma (volutrauma) and on continuous reopening and collapsing of lung parenchyma through the generation of shear forces. Until now, the used techniques in our study do not offer the possibility to detect hyperinflation. Further studies using EIT might inaugurate more advanced algorithms for detection of hyperinflation.

\section{Conclusion}

The results show ventilation redistribution during mechanical ventilation from non-ventilated regions to bad ventilated regions as well as to moderately and well-ventilated regions by increasing the PEEP. These findings are consistent with results obtained by lung CT scan studies. However, EIT is a bedside technique and might be an alternative to CT scan to assess aerated lung regions.

\section{Acknowledgements}

This study was supported by departmental funds and by Dräger AG (Dräger AG, Lübeck, Germany).

\section{References}

1. Poulton EP. Left sided heart failure with pulmonary edema. Its treatment with the 'pulmonary plus pressure machine'. Lancet 1936; 2: 981-994.

2. Ashbaugh DG, Petty TL, Bigelow DB, Harris TM. Continuous positive-pressure breathing (CPPB) in adult respiratory distress syndrome. J Thorac Cardiovasc Surg 1969; 57: 31-41.

3. Mancebo J. PEEP, ARDS, and alveolar recruitment. Intens Care Med 1992; 18: 383-385.
4. Suter PM, Fairley B, Isenberg MD. Optimum end-expiratory airway pressure in patients with acute pulmonary failure. New Engl J Med 1975; 292: 284-289.

5. Dammann JF, McAslan TC. PEEP: its use in young patients with apparently normal lungs. Crit Care Med 1979; 7: $14-19$.

6. Kumar A, Falke KJ, Geffin B et al. Continuous positivepressure ventilation in acute respiratory failure. New EnglJ Med 1970; 283: 1430-1436.

7. Chapin JC, Downs JB, Douglas ME et al. Lung expansion, airway pressure transmission, and positive end-expiratory pressure. Arch Surg 1979; 114: 1193-1197.

8. Moreci AP, Norman JC. Measurements of alveolar sac diameters by incident-light photomicrography. Effects of positivepressure respiration. Ann Thorac Surg 1973; 15: 179-186.

9. McIntyre RW, Laws AK, Ramachandran PR. Positive expiratory pressure plateau: improved gas exchange during mechanical ventilation. Can Anaesth Soc J 1969; 16: 477-486.

10. Katz JA, Ozanne GM, Zinn SE, Fairley HB. Time course and mechanisms of lung-volume increase with PEEP in acute pulmonary failure. Anesthesiology 1981; 54: 9-16.

11. Dreyfuss D, Saumon G. Ventilator-induced lung injury: lessons from experimental studies. Am J Respir Crit Care Med 1998; 157: 294-323.

12. Gattinoni L, Pelosi P, Crotti S, Valenza F. Effects of positive end-expiratory pressure on regional distribution of tidal volume and recruitment in adult respiratory distress syndrome. Am J Respir Crit Care Med 1995; 151: 1807-1814.

13. Puybasset L, Gusman P, Muller JC et al. Regional distribution of gas and tissue in acute respiratory distress syndrome. III. Consequences for the effects of positive end-expiratory pressure. CT Scan ARDS Study Group. Adult Respiratory Distress Syndrome. Intens Care Med 2000; 26: 1215-1227.

14. Rouby JJ, Lu Q, Goldstein I. Selecting the right level of positive end-expiratory pressure in patients with acute respiratory distress syndrome. Am J Respir Crit Care Med 2002; 165: 1182-1186.

15. Barber DC, Brown BH. Applied potential tomography. J Phys E Sci Instrum 1984; 17: 723-733.

16. Victorino JA, Borges JB, Okamoto VN et al. Imbalances in regional lung ventilation: a validation study on electrical impedance tomography. Am J Respir Crit Care Med 2004; 169: 791-800.

17. Adler A, Shinozuka N, Berthiaume Y et al. Electrical impedance tomography can monitor dynamic hyperinflation in dogs. J Appl Physiol 1998; 84: 726-732.

18. Adler A, Amyot R, Guardo R et al. Monitoring changes in lung air and liquid volumes with electrical impedance tomography. J Appl Physiol 1997; 83: 1762-1767.

19. Kunst PW, Bohm SH, de Vazquez A et al. Regional pressure volume curves by electrical impedance tomography in a model of acute lung injury. Crit Care Med 2000; 28: $178-183$.

20. Kunst PW, de Vazquez A, Bohm SH et al. Monitoring of recruitment and derecruitment by electrical impedance tomography in a model of acute lung injury. Crit Care Med 2000; 28: 3891-3895.

21. Darling RC, Richards DW, Cournant A. Studies on intrapulmonary mixture of gases. Open circuit method for measuring residual air. J Clin Invest 1940; 19: 609-618. 
22. Wrigge $\mathrm{H}$, Sydow M, Zinserling J et al. Determination of functional residual capacity (FRC) by multibreath nitrogen washout in a lung model and in mechanically ventilated patients. Accuracy depends on continuous dynamic compensation for changes of gas sampling delay time. Intens Care Med 1998; 24: 487-493.

23. Barber DC, Seagar AD. Fast reconstruction of resistance images. Clin Phys Physiol Meas 1987; 8(Suppl A): 47-54.

24. Frerichs I, Hinz J, Herrmann $P$ et al. Detection of local lung air content by electrical impedance tomography compared with electron beam CT. J Appl Physiol 2002; 93: 660-666.

25. Hahn G, Sipinkova I, Baisch F, Hellige G. Changes in the thoracic impedance distribution under different ventilatory conditions. Physiol Meas 1995; 16: A161-A173.

26. Frerichs I. Electrical impedance tomography (EIT) in applications related to lung and ventilation: a review of experimental and clinical activities. Physiol Meas 2000; 21: R1-R21.

27. Hinz J, Neumann P, Dudykevych T et al. Regional ventilation by electrical impedance tomography - a comparison with ventilation scintigraphy in pigs. Chest 2003; 124: 314-322.

28. Arnold JH. Electrical impedance tomography: on the path to the holy grail. Crit Care Med 2004; 32: 894-895.

29. Hinz J, Hahn G, Neumann P et al. End-expiratory lung impedance change enables bedside monitoring of endexpiratory lung volume change. Intens Care Med 2003; 29: 37-43.

30. Wolf GK, Arnold JH. Noninvasive assessment of lung volume: respiratory inductance plethysmography and electrical impedance tomography. Crit Care Med 2005; 33: S163-S169.

31. Frerichs I, Schiffmann H, Oehler R et al. Distribution of lung ventilation in spontaneously breathing neonates lying in different body positions. Intens Care Med 2003; 29: 787-794.

32. Hahn G, Hartung C, Hellige G (1998) Bestimmung der Grösse minimal erfassbarer Areale mit Ventilationsstörungen. p. 77

33. Brown $\mathrm{BH}$, Barber DC. Electrical impedance tomography: the construction and application to physiological measurement of electrical impedance images. Med Prog Technol 1987; 13: 69-75.

34. Koukourlis CS, Kyriacou GA, Sahalos JN. A 32-electrode data collection system for electrical impedance tomography. IEEE Trans Biomed Eng 1995; 42: 632-636.

35. Li JH, Joppek C, Faust U. Fast EIT data acquisition system with active electrodes and its application to cardiac imaging. Physiol Meas 1996; 17(Suppl 4A): A25-A32.
36. Weisser G, Lehmann KJ, Scheck R et al. Performance of electron-beam CT: continuous-volume-scan compared to spiral CT. Radiologe 1998; 38: 993-998.

37. Weisser G, Lehmann KJ, Scheck R et al. Dose and image quality of electron-beam CT compared with spiral CT. Invest Radiol 1999; 34: 415-420.

38. Burch WM, Sullivan PJ, Lomas FE et al. Lung ventilation studies with technetium-99 m pseudogas. J Nucl Med 1986; 27: 842-846.

39. Wrigge $\mathrm{H}$, Zinserling J, Neumann $\mathrm{P}$ et al. Spontaneous breathing improves lung aeration in oleic acid-induced lung injury. Anesthesiology 2003; 99: 376-384.

40. Faes TJ, van der Meij HA, de Munck JC, Heethaar RM. The electric resistivity of human tissues $(100 \mathrm{~Hz}-10 \mathrm{MHz})$ : a meta-analysis of review studies. Physiol Meas 1999; 20: $\mathrm{R} 1-\mathrm{R} 10$

41. Wtorek J, Polinski A. The contribution of blood-flowinduced conductivity changes to measured impedance. IEEE Trans Biomed Eng 2005; 52: 41-49.

42. Hahn G, Frerichs I, Kleyer M, Hellige G. Local mechanics of the lung tissue determined by functional EIT. Physiol Meas 1996; 17(Suppl 4A): A159-A166.

43. Hahn G, Thiel F, Dudykevych T et al. Quantitative evaluation of the performance of different electrical tomography devices. Biomed Tech (Berlin) 2001; 4: 91-95.

44. Lim CM, Soon LS, Seoung LJ et al. Morphometric effects of the recruitment manoeuvre on saline-lavaged canine lungs. A computed tomographic analysis. Anesthesiology 2003; 99: 71-80.

45. Hedenstierna G, Strandberg A, Brismar B et al. Functional residual capacity, thoracoabdominal dimensions, and central blood volume during general anesthesia with muscle paralysis and mechanical ventilation. Anesthesiology 1985; 62: 247-254.

46. Gattinoni L, D’Andrea L, Pelosi P et al. Regional effects and mechanism of positive end-expiratory pressure in early adult respiratory distress syndrome. JAMA 1993; 269: 2122-2127.

47. Frerichs I, Hahn G, Golisch W et al. Monitoring perioperative changes in distribution of pulmonary ventilation by functional electrical impedance tomography. Acta Anaesthesiol Scand 1998; 42: 721-726. 


\title{
Regional pulmonary pressure volume curves in mechanically ventilated patients with acute respiratory failure measured by electrical impedance tomography
}

\author{
J. Hinz, O. Moerer, P. Neumann, T. Dudykevych, I. Frerichs, G. Hellige and M. Quintel \\ Department of Anaesthesiology, Emergency and Intensive Care Medicine, University of Goettingen, Goettingen, Germany
}

\begin{abstract}
Background: We hypothized, that in mechanically ventilated patients with acute respiratory failure, regional pressure volume curves differ markedly from conventional global pressure volume curves of the whole lung.

Methods: In nine mechanically ventilated patients with acute respiratory failure during an inspiratory low-flow manoeuvre, conventional global pressure volume curves were registered by spirometry and regional pressure volume curves in up to 912 regions were assessed simultaneously using electrical impedance tomography. We compared the lower (LIP) and upper (UIP) inflection points obtained from the conventional global pressure volume curve and regional pressure volume curves.

Results: We identified from the conventional global pressure volume curves LIP [3-11 (8) $\mathrm{cmH}_{2} \mathrm{O}$ ] in eight patients and UIP [31-39 (33) $\mathrm{cmH}_{2} \mathrm{O}$ ] in three patients. Using electrical impedance tomography (EIT), LIP [3-18 (8) $\left.\mathrm{cmH}_{2} \mathrm{O}\right]$ in 54-264 (180) regions and UIP [23-42 (36) $\mathrm{cmH}_{2} \mathrm{O}$ ] in 149-324 (193) regions (range and
\end{abstract}

median) were identified. Lung mechanics measured by conventional global pressure volume curves are similar to the median of regional pressure volume curves obtained by EIT within the tomographic plane. However, single regional pressure volume curves differ markedly with a broad heterogeneity of lower and upper inflection points.

Conclusion: Lower and upper inflection points obtained from conventional global pressure volume curves are not representative of all regions of the lungs.

Accepted for publication 30 October 2005

Keywords: electrical impedance tomography; lung mechanics; pressure volume curves; regional.

C) 2006 The Authors

Journal compilation (C) 2006 Acta Anaesthesiol Scand
$I^{2}$ T was proposed that ventilator settings should be optimized by conventional pressure volume (PV) curves of the lung (1). Amato and co-workers showed a better survival of mechanically ventilated patients using a protective ventilatory strategy guided by PV curves (2). However, PV curves obtained from the whole lung may not be representative of the respiratory mechanics of different lung regions. It was shown in animal studies with lavage-induced lung injury that regional PV curves measured by electrical impedance tomography (EIT) differ from conventional PV curves (3-5). Recently, differences in regional compliances were measured in a large series of acute respiratory distress syndrome (ARDS) patients (6). Regional inhomogeneities of the diseased lung should be considered to optimize ventilatory settings in patients with ARDS or acute lung injury (ALI). Although, this review pointed out that the problem of setting the right positive endexpiratory pressure (PEEP) according to an inspiratory or expiratory PV curve remains unsolved (7). A study in mechanically ventilated patients with early lung injury showed, that the use of an expiratory PV curve for a PEEP setting in patients might be beneficial $(8,9)$.

Therefore, we hypothesized in mechanically ventilated patients with acute respiratory failure that inspiratory PV curves from one lung plane measured by EIT provide information similar to that provided by conventional inspiratory PV curves. Additionally, we hypothesized that information from regional inspiratory PV curves measured by EIT shows a broad heterogeneity.

\section{Materials and methods}

\section{Study protocol}

After approval by the local ethics committee, we performed the investigation in the anaesthesiological intensive care unit of the university hospital in Goettingen, Germany. Informed consent for participation in the study was obtained from the next of 


\section{J. Hinz et al.}

kin from nine mechanically ventilated patients with acute respiratory failure (ARF). We simultaneously measured conventional PV curves of the whole lung by spirometry $\left(\mathrm{PV}_{\text {Spirometry }}\right), \mathrm{PV}$ curves in one transversal plane of the thorax by EIT (PV EITglobal) and regional PV curves (PV EITregional) by EIT in up to 912 regions of interest (ROI). We compared the lower inflection point (LIP) and the upper inflection point (UIP) obtained from $\mathrm{PV}_{\text {Spirometry, }} \mathrm{PV}_{\text {EITglobal }}$ and $\mathrm{PV}_{\mathrm{EITregional}}$, respectively. During PV measurements, continuous sedation with intravenous propofol was provided to achieve a Ramsay score of 4-5 (10) and muscle relaxation was performed with pancuronium bromide (Pancuronium; Organon, BH Oss, the Netherlands). For patients characteristics see Table 1.

Inclusion criteria were: (i) mechanically ventilated patient with ARF $\left(\mathrm{PaO}_{2} / \mathrm{F}_{\mathrm{I}} \mathrm{O}_{2}<300 \mathrm{mmHg}=\right.$ $40 \mathrm{kPa}$ ) (11); (ii) age $\geq 18$ years; and (iii) clinically indicated arterial blood pressure measurement.

Exclusion criteria were: (i) implanted electrical devices (i.e. cardiac pacemaker) [no data are available yet how highly conductive material inserted into the thorax (e.g. pacemaker, sternal wire cerclage) may affect or is affected by EIT measurements]; (ii) chronic obstructive lung disease (suspicion or history); (iii) intrinsic PEEP $>2.5 \mathrm{cmH}_{2} \mathrm{O}$; (iv) puncture of the subclavia vein $<4 \mathrm{~h}$ before onset of the study; (v) unstable haemodynamics (high doses of epinephrine, norepinephrine or dobutamine); (vi) pneumothorax/ mediastinal emphysema; (vii) pregnancy; and (viii) terminal illness.

\section{Mechanical ventilation}

For mechanical ventilation a respirator (EVITA 4; Dräger AG, Lübeck, Germany) was used. Thirty minutes before measurement, ventilation was set at a volume-controlled mode with a tidal volume of $8 \mathrm{ml} / \mathrm{kg}$. Respiratory rate was adjusted to reach an arterial $\mathrm{PaCO}_{2}<55 \mathrm{mmHg}=7.33 \mathrm{kPa}$. PEEP and inspiratory oxygen were adjusted to reach an oxygen saturation $>90 \%$. We adjusted the inspiratory/ expiratory ratio to minimize end-expiratory flow in order to avoid intrinsic PEEP, which was measured by end-expiratory occlusion of the airways and pressure measurement incorporated in the ventilator. After baseline ventilation, $5 \mathrm{~min}$ of ventilation was performed without PEEP. Thereafter, PV curves were registered during zero PEEP conditions.

\section{Pressure volume curves}

During an inspiratory low flow manoeuvre, PV curves were measured (12) using a gas flow of $21 / \mathrm{min}$ provided by the ventilator, which was controlled using a laptop (Toshiba Satellite Pro 4200 series, Pentium II, 233 MHZ, 32 MB RAM; Windows NT, Toshiba Europe Gmbtt, Neuss, Germany) running custom-made software (Evita4Lab; Dräger AG, Lübeck, Germany). We used identical breathing circuits (Intersurgical Complete Respiratory Systems, Wokingham, UK) for all patients to keep their influence on compliance constant. During zero PEEP conditions, registration of PV curves was started after a long expiratory pause in order to avoid intrinsic PEEP. During zero PEEP conditions, the inspiratory oxygen was adjusted to reach an oxygen saturation $>90 \%$. For safety reasons, airway pressure was limited to $46 \mathrm{cmH}_{2} \mathrm{O}$ during the low flow manoeuvre. Because low flow minimizes the influence of the airway resistance on respiratory mechanics, we

\section{Table 1}

\begin{tabular}{|c|c|c|c|c|c|c|c|c|c|}
\hline \multicolumn{10}{|c|}{ Patients' characteristics } \\
\hline 1 & 50 & 100 & 162 & pneumonia & pneumonia & bilateral & 8 & 1.75 & 39 \\
\hline 2 & 34 & 90 & 185 & skull brain injury & pneumonia & bilateral & 10 & 2.00 & 25 \\
\hline 3 & 50 & 85 & 175 & skull brain injury & pneumonia & bilateral & 6 & 1.75 & 31 \\
\hline 4 & 76 & 95 & 180 & pneumonia & Pneumonia & unilateral & 12 & 2.00 & 30 \\
\hline 7 & 38 & 75 & 175 & epidural haematoma & pneumonia & bilateral & 10 & 2.50 & 19 \\
\hline 8 & 42 & 70 & 175 & liver cirrhosis & pneumonia & bilateral & 10 & 2.33 & 35 \\
\hline 9 & 56 & 85 & 167 & skull brain injury & pneumonia & unilateral & 12 & 2.00 & 37 \\
\hline Min-Max & $34-76$ & $70-100$ & $162-185$ & & & & $6-12$ & $1.5-2.5$ & $19-40$ \\
\hline (Median) & (50) & (85) & $(175)$ & & & & $(10)$ & $(2.0)$ & (35) \\
\hline
\end{tabular}

LIS, lung injury score (19); SAB, subarachnoidal haemorrhage.

The patients were ventilated with positive end expiratory pressure (PEEP) set by the attending physician before inclusion in the study. 
assumed that regional pressures were similar to the airway pressure at the airway opening.

\section{Conventional PV curves}

Conventional PV curves ( $\left.\mathrm{PV}_{\text {Spirometry }}\right)$ were constructed by spirometry and airway pressure measurement. Spirometry was performed using a pneumotachograph (flow head: Fleisch no. 2, Fleisch, Lausanne, Switzerland; differential pressure transducer: Huba Control, Würenlos, Switzerland) directly connected to a heat and moisture exchanger at the endotracheal tube. Airway pressure was measured using a pressure transducer (Druckwandler $\mathrm{AP}$, SI-special instruments $\mathrm{GmbH}$, Nördlingen, Germany) connected via a capillary to the heat and moisture exchanger.

\section{EIT PV curves}

EIT was presented in detail elsewhere $(13,14)$. During the low flow manoeuvre, we obtained EIT PV curves from impedance change derived from EIT and airway pressure at the airway opening. The boundaries of the lungs were determined from the functional EIT ventilation images (f-EIT) (15). The impedance variance of the lungs is higher compared with other thoracic structures (i.e. chest wall, mediastinum) as a result of the great impedance changes associated with air content (16). The chest wall contributes solely to the outer boundary of the f-EIT image. The mean impedance variation of this boundary was set as the limit to define the 'EIT lung regions'. Because of this approach, the number of regions observed by EIT varies from patient to patient. We calculated PV curves from the EIT plane (6th intercostal parasternal space) of the lung ( $\left.P V_{\text {EITglobal }}\right)$. Additionally, within this plane in regions defined as the 'EIT lung region' (impedance variation $>$ mean impedance of outer boundary), up to 912 regional EIT PV curves were reconstructed $\left(\mathrm{PV}_{\mathrm{EITregional}}\right)$. Figure 1 shows six examples out of 230 regional PV curves of a mechanically ventilated patient.

\section{Evaluation of $P V$ curves}

To avoid inter-observer variability (17), lower (LIP) and upper (UIP) inflection points were calculated from fittings to $\mathrm{PV}_{\text {Spirometry, }} \mathrm{PV}_{\mathrm{EITglobal}}$ and $\mathrm{PV}_{\mathrm{EITregional}}$ to a sigmoid equation $\mathrm{V}=\mathrm{b} /\left(1+\mathrm{e}^{-(\mathrm{P}-\mathrm{c}) / \mathrm{d}}\right)$ described by Venegas and co-workers (18) (Fig. 2). Fittings were performed using commercially available mathematical software (MatLab 6.0; The MathWorks, Natick, MA). We checked the quality of fittings by simultaneously plotting the measured and fitted data. If the square of the correlation coefficient $\left(R^{2}\right)$ was above 0.90 the fitting was accepted.

Compliance was calculated according to the equation $\mathrm{C}=\mathrm{dV} / \mathrm{dP}$ (with $\mathrm{C}$ : compliance; $\mathrm{dV}$ : volume change; $\mathrm{dP}$ : pressure change) from the slope of a conventional inspiratory PV curve at the point of highest compliance. The point of highest compliance was calculated from the parameter c, as proposed by Venegas and co-worker (18).

\section{Haemodynamics and gas exchange}

Arterial blood was sampled via a 20-gauge catheter radial or femoral artery and analysed using $\mathrm{ABL}$ 300 and an OSM 3 Hemoximeter (Radiometer, Copenhagen, Denmark). ECG, systemic arterial and central venous pressures were displayed on a bedside monitor together with the oxyhaemoglobin saturation (Datex AS/3; Datex Divison Instrumentarium Corp., Helsinki, Finland) and recorded with reference to atmospheric pressure at the mid-thoracic level at end-expiration.

\section{Statistics}

Calculations were performed using the STATISTICA software package (Statistica 5.1; StatSoft Inc., Tulsa, $\mathrm{OK})$. We tested normal distribution using the Kolmogorov-Smirnov test. All data are presented as Min-Max (Median) unless stated otherwise. We applied a Wilcoxon-matched pair test to analyse differences between the two methods. Linear regression analysis, using the least square method, was applied for correlation analysis. For all statistical tests, $P<0.05$ was considered to be significant.

\section{Results}

We investigated nine mechanically ventilated patients with acute respiratory failure. The reasons for respiratory failure were pneumonia in seven patients, whereas two patients had atelectasis. The median lung injury score (19) was 2.0 and the $\mathrm{PaO}_{2} /$ $\mathrm{F}_{\mathrm{I}} \mathrm{O}_{2}$ was 143-298 (median 260) mmHg (19-40 (median 35) $(\mathrm{kPa})$. Compliance varied from 30 to 82 (median 51) $\mathrm{ml} / \mathrm{cmH}_{2} \mathrm{O}$. Before inclusion in the study, the patients were ventilated with PEEP set by the attending physician from 6 to 12 (median 10) $\mathrm{cmH}_{2} \mathrm{O}$. No side-effects occurred during the low-flow manoeuvre, which stopped at an airway pressure above $45 \mathrm{mmHg}=6 \mathrm{kPa}$. During this manoeuvre, the oxyhaemoglobin saturation $\left(\mathrm{SaO}_{2}\right)$ was stable. It was 98-100 (99)\% directly before the low-flow manoeuvre and 98-100 (99)\% directly after the manoeuvre. In addition, the patients showed no 


\section{J. Hinz et al.}

Ventral left

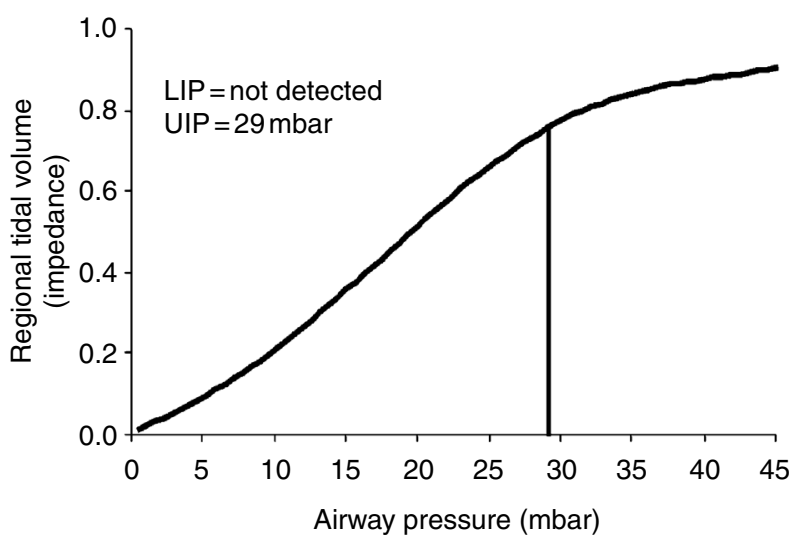

Median left

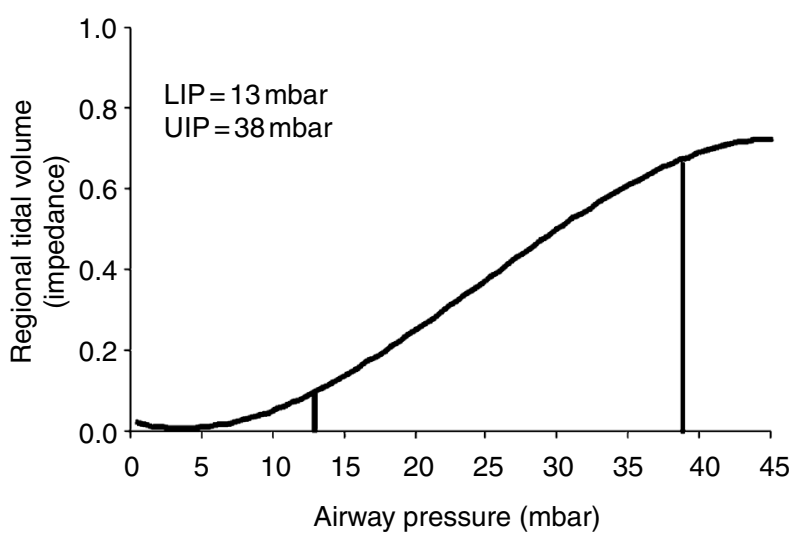

Dorsal left

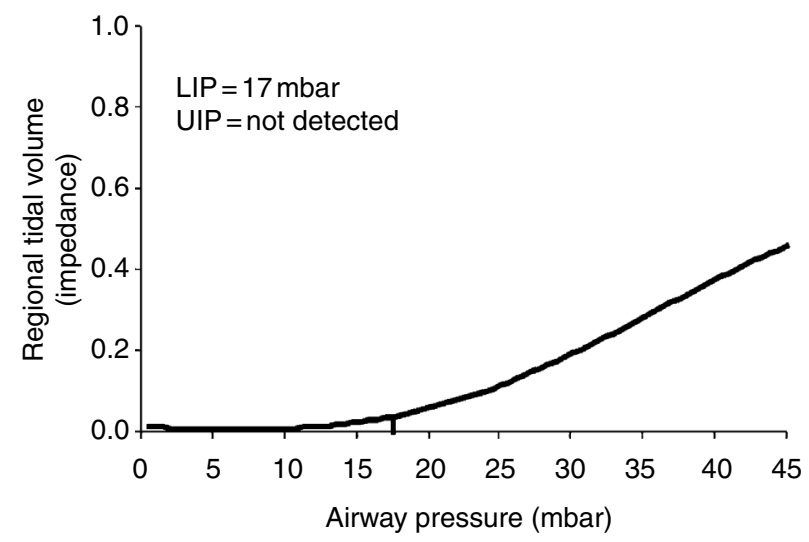

Ventral right
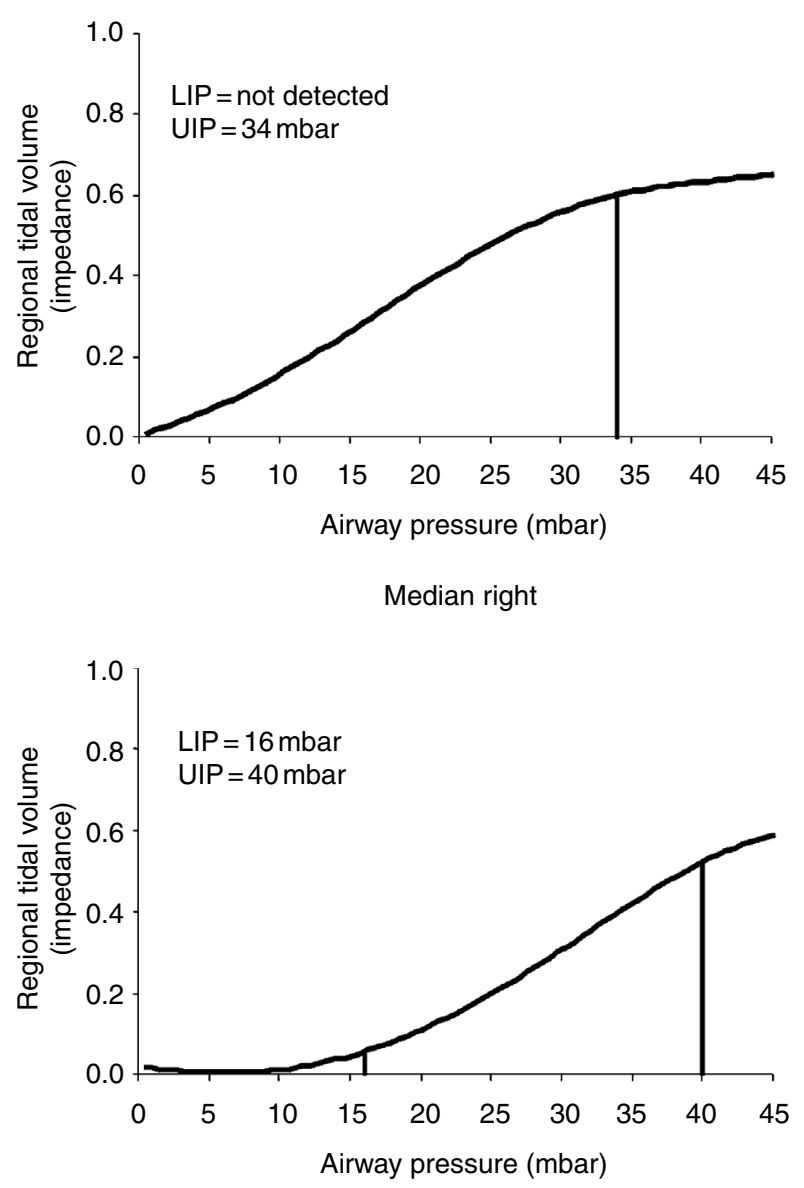

Dorsal right

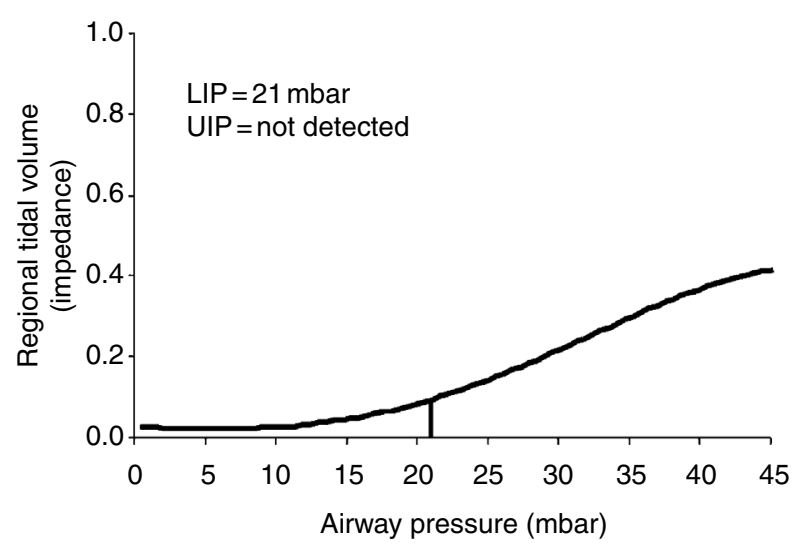

Fig. 1. Six examples out of 230 different regional pressure volume (PV) curves obtained using electrical impedance tomography (EIT) and airway pressure measurement during a low-flow manoeuvre in one mechanically ventilated patient suffering from pneumonia. Impedance change was determined in six different regions of interest (dorsal, ventral, median, left and right) consisting each of one EIT region showing a marked heterogeneity in the slope, lower (LIP) and upper (UIP) inflection points of the regional PV curves.

alterations in haemodynamics during the low-flow manoeuvre, with a mean arterial blood pressure of 70-107 (95) $\mathrm{mmHg}$ measured immediately before the manoeuvre and 74-110 (97) $\mathrm{mmHg}$ directly after the manoeuvre. For patients summary see Table 1 and Table 2.

For all patients, the normalized global tidal volumes inhaled during the manoeuvre correlated 


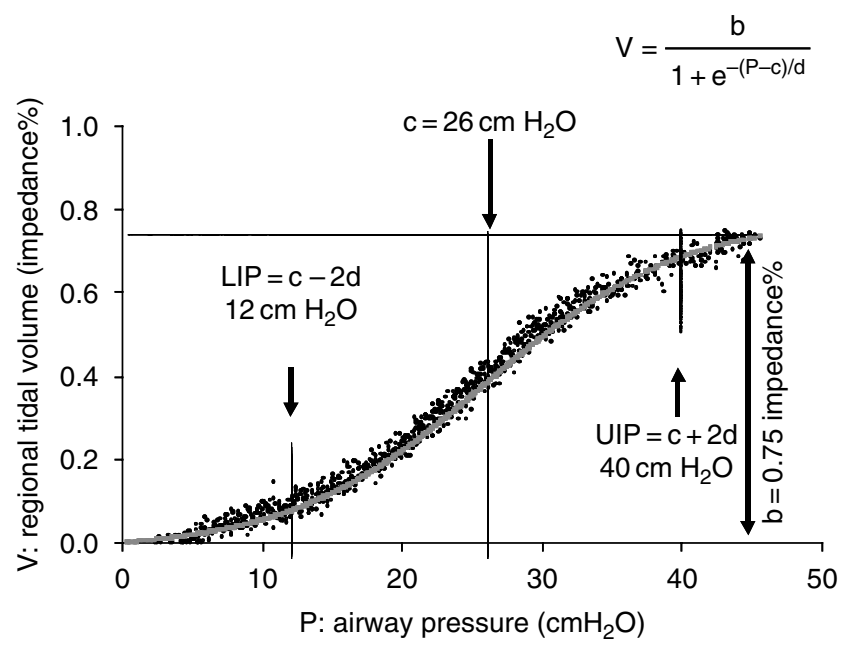

Fig. 2. Evaluation of pressure volume $(P V)$ curves. Example of a regional PV curve measured by electrical impedance tomography (EIT) during an inspiratory low-flow manoeuvre in a mechanically ventilated patient and fitted to the equation $V=b /\left(1+e^{-(P-c) / d}\right)$. In this equation, $V$ is the tidal volume above end expiratory lung volume, and $P$ is the airway pressure. Fitting parameters are $b, c$ and $d$. Each of these parameters has a physiological correlate: $b$ corresponds to the volume of the upper asymptote of the PV curve, $c$ corresponds to the pressure at the point of highest compliance of the PV curve and $d$ is a width parameter proportional to the pressure range in which most of the volume changes occurs. The lower inflection point (LIP) is calculated as LIP $=c-2 d$, while the upper inflection point (UIP) is calculated as UIP $=c+2 d$.

significantly with impedance changes measured by EIT within this plane $\left(y=1.06 x-7.4, r^{2}=0.96\right)$. Because of the procedure of lung regions detection, the limits of impedance change defining the EIT lung regions were above $0.01-0.10$ (0.02) which were $2.75 \%-8.91 \%(3.68 \%)$ per cent of the maximal observed impedance change in each patient. Therefore the number of regions, in which regional PV curves using EIT were observed, varies from patient to patient. We investigated 445-682 (Median 573) lung regions out of 912 possible EIT regions, which was $63 \%$ of the measured thoracic EIT plane.

\section{Comparison of LIP and UIP}

We constructed nine conventional PV curves, nine global EIT PV curves and 4512 regional EIT PV curves. We fitted these 4530 PV curves to the sigmoid equation with excellent precision of $r^{2}$ 0.93-0.98 (median 0.95). We summarized all results from the LIP and UIP of the global and regional measurements in Table 2 and Fig. 3.

\section{Comparison of LIP}

Using global measurements of conventional and EIT PV curves within the plane ( $\left.\mathrm{PV}_{\text {Spirometry, }} \mathrm{PV}_{\text {EITglobal }}\right)$, LIP were identified in eight out of nine patients. We found LIP varying from 3 to 11 (median 8) $\mathrm{cmH}_{2} \mathrm{O}$ in the conventional and from 4 to 12 (median 7) $\mathrm{cmH}_{2} \mathrm{O}$ in the EIT PV curves. LIP derived from both global measurements showed a close linear correlation $\left(y=1.05 x+0.03, r^{2}=0.93\right)$. However, in the regional measurements ( $\left.\mathrm{PV}_{\text {EITregional }}\right)$ there were a broad intra-individual heterogeneity of LIP regarding the pressures and numbers of regions. We found LIP from the intra-individual regional measurements in

Table 2

Compliance and inflection points

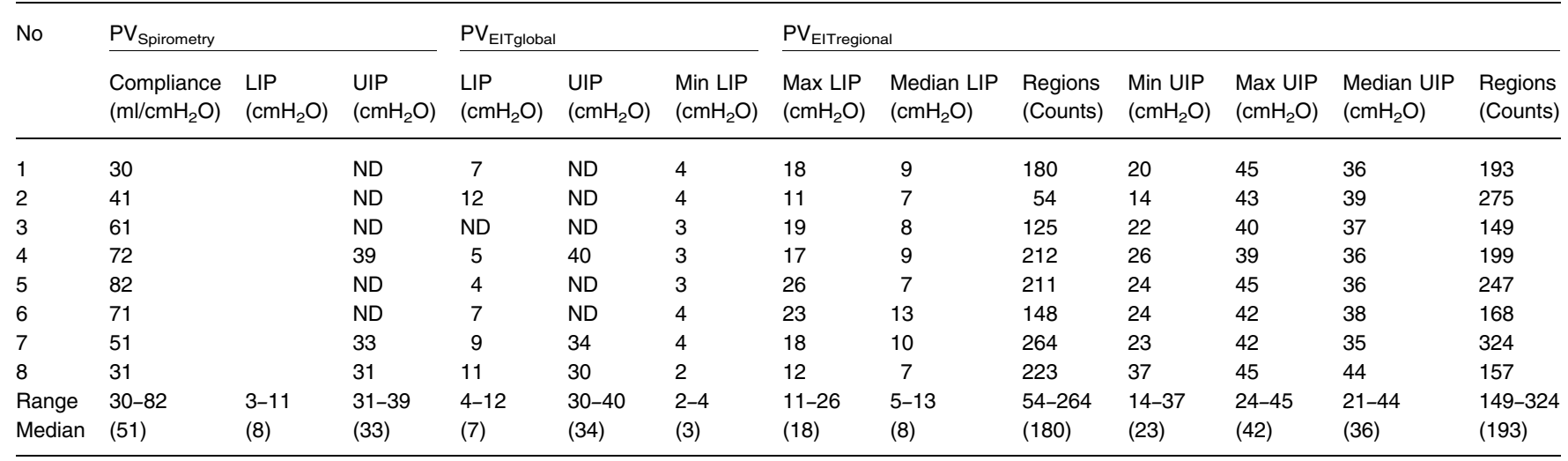

Lower inflection point (LIP) and upper inflection points (UIP) from pressure volume curves in mechanically ventilated patients with acute respiratory failure.

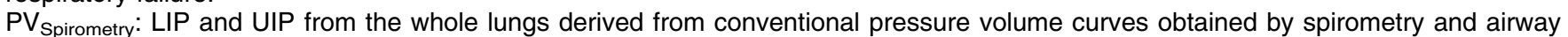

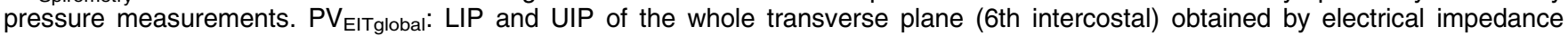
tomography (EIT) and airway pressure measurements. PV EITregional: LIP and UIP from regional pressure volume curves obtained by electrical impedance tomography (EIT) and airway pressure measurement in the 6th intercostal thoracic plane. ND, not detected. 

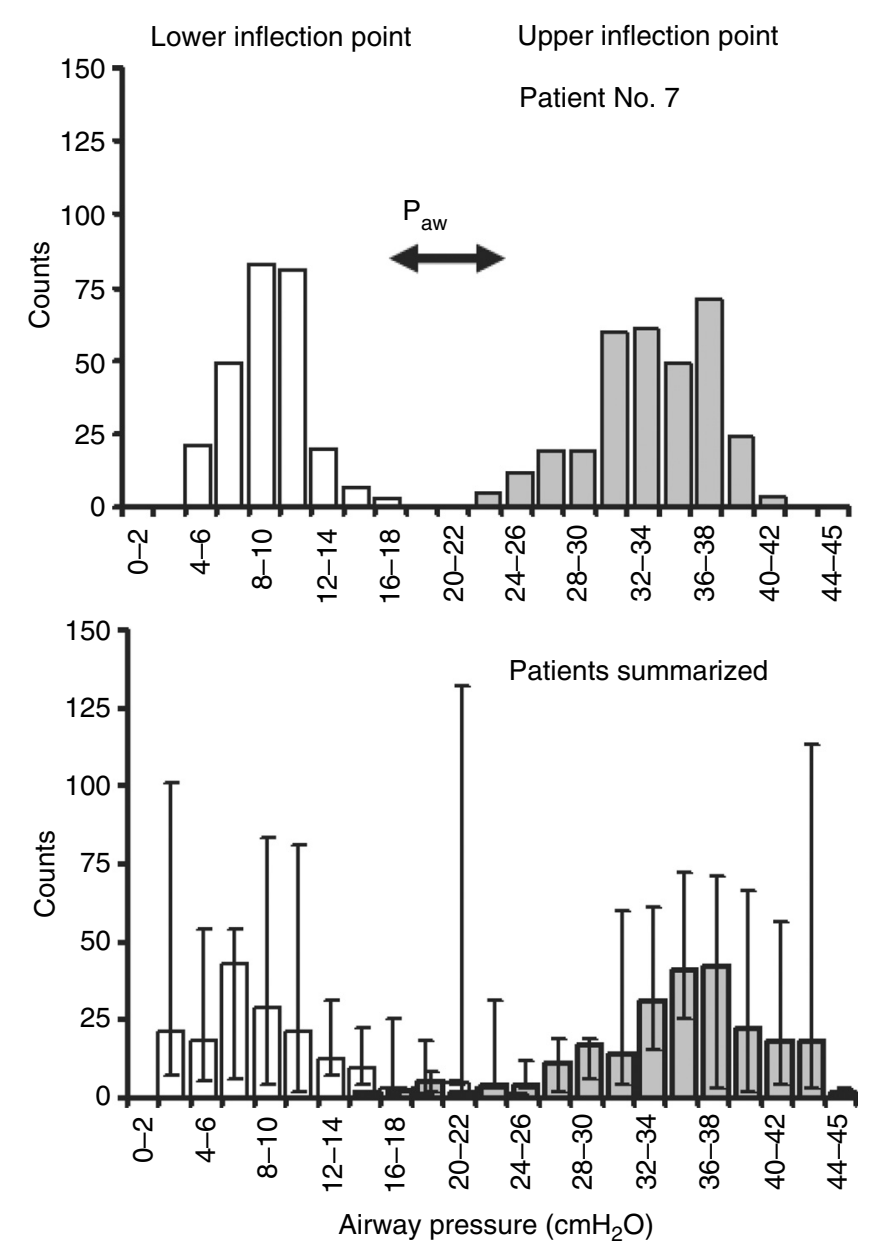

Fig. 3. Example of histographic presentation of lower (LIP) and upper (UIP) inflection points in a transverse thoracic plane in one mechanically ventilated patient and nine patients summarized constructed by electrical impedance tomography (EIT) and airway pressure measurement. Note, in the example of patient 7 , the distribution of regional lower and upper inflection points may offer the possibility to adjust the ventilatory setting, so that end expiratory lung collapse and end inspiratory over distension can be avoided in the majority of lung regions $P_{a w}$.

54-264 (median 180) regions and they varied from 2 to 26 (median 8) $\mathrm{cmH}_{2} \mathrm{O}$. The median values of the intra-individual regional LIP were similar to LIP derived from the global measurements.

\section{Comparison of UIP}

We identified UIP in the global measurements in three patients. The upper inflection point from the conventional PV curves varied from 31 to 39 (median 33) $\mathrm{cmH}_{2} \mathrm{O}$ and from the EIT PV curves from 30 to 40 (median $34 \mathrm{cmH}_{2} \mathrm{O}$ ). Again, we found a close linear correlation between global UIP derived from spirometry and EIT $\left(y=1.19 x-6.08, R^{2}=0.97\right)$. However, similar to the lower inflection points, the UIP obtained from the regional measurements
$\left(\mathrm{PV}_{\text {EITregional }}\right)$ were quite heterogeneous regarding the pressure and number of regions, in which they were identified. We investigated UIP from the regional measurements which varied from 14 to 44 (median 36) $\mathrm{cmH}_{2} \mathrm{O}$ in 149-324 (193) regions. The median values of the UIP from these regional measurements were similar to the upper inflection point derived from the global measurements.

\section{Discussion}

We intended to investigate the diversity of regional lung mechanics measuring LIP and UIP from regional pressure volume curves in mechanically ventilated patients suffering from ARF and found a broad heterogeneity of the regional EIT PV curves. Until the latest studies $(8,20,21)$, it was assumed that the appropriate positive end-expiratory pressure keeping the lung open is determined from the LIP of a conventional PV curve. The UIP of the PV curve should not be exceeded by the inspiratory plateau pressure to avoid over distension of the lung (22). However, conventional PV curves are a global measurement of the total lung and influenced by regional lung mechanic characteristics $(3,8,20)$. Due to this mechanism, the lung itself is an active trigger in the multiple-organ failure sequence (23-25).

\section{PV curves}

Global lung mechanics from $P V_{\text {Spirometry }}$ vs. $P V_{\text {EITglobal }}$ We found a correlation of the LIP calculated from the global PV curves obtained by spirometry and in the observed thoracic plane. Similar results were found by Kunst and co-workers in a surfactantdepleted lung injury model in pigs. A similar correlation of upper inflection points measured by conventional PV curves and in the studied thoracic plane using EIT was also found in our study and in the study by Kunst and co-worker (3). However, LIP and UIP could not be detected in all patients from the conventional PV curve and the EIT PV curve of the thoracic plane. Similar results were found by Sydow and co-workers $(26,27)$.

Regional lung mechanics from $P V_{\text {EITregional }}$

We identified a broad heterogeneity of pressures and quantities of regional lower and upper inflection points (Table 2 and Fig. 3). Additionally, we found a gravity-dependent increase in the regional LIP (Figs 4 and 5). These results may explain the results of Hickling, who showed in a mathematical model of an ARDS lung, that recruitment of the 

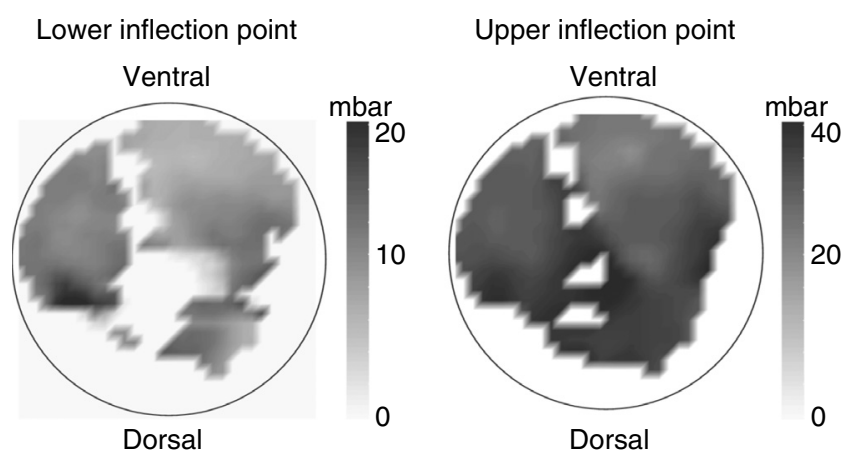

Fig. 4. Tomogram of a transverse thoracic slice showing the regional distribution of lower (LIP) and upper (UIP) inflection points and the corresponding pressure values in a mechanically ventilated patient suffering from pneumonia measured by electrical impedance tomography (EIT) and airway measurement.

whole lung occurs along the entire conventional PV curve (21).This is in agreement with the study performed by Kunst (3), who found a significant higher LIP in the dorsal part compared with the anterior part of the lung. Aeration gradients are found not only gravity dependent but also in the cephalo-caudal direction $(6,28)$. Therefore, during measurements the lungs may displace in the caudal direction, so that EIT is constantly measuring different lung planes.

Our results are comparable to those of Gattinoni $(29,30)$ and Pelosi (31), although studied using computed tomography. Computed tomography generates static images with morphological information with respect to the tissue density. In contrast, EIT monitors the dynamic of impedance change in

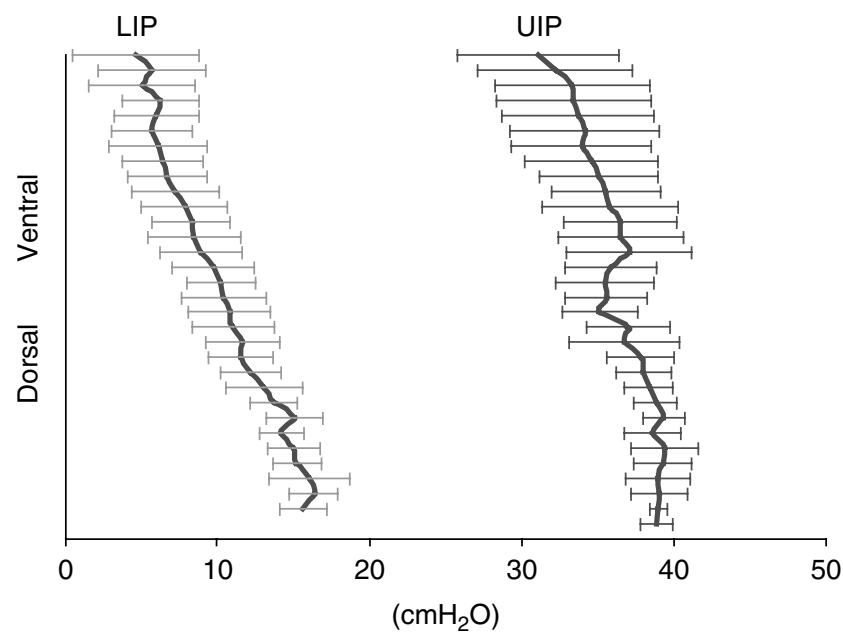

Fig. 5. Regional lower (LIP) and upper (UIP) inflection points of nine mechanically ventilated patients in the dorsal to ventral direction in an approximate 3-cm slice of the thorax measured by electrical impedance tomography (EIT) (Means and standard deviation). different thoracic regions. The findings of our study may explain the results published by Crotti and coworkers, that recruitment occurs along the entire PV curve (20). Furthermore, hyperinflation occurs before UIP and is sustained beyond UIP. This indicates that inflection points from conventional PV curves do not reflect the regional lung mechanics (30).

The reconstruction of regional inflection points depend on the spatial and temporal resolution (32). In the study performed by Kunst and co-workers (3), they used a spatial resolution of two regions of interest (anterior and posterior part of the lungs) whereas we used the resolution of up to 912 EIT PV curves. Additionally, inflection points are overestimated by low sample frequency. Kunst and co-workers averaged 10 EIT cycles generating one EIT image per second, whereas we measured 13 EIT images per second.

\section{Implications for respiratory therapy}

Several authors pointed out the regional information provided by EIT, especially how much this information differs from global measurements (4, 33-36). In our study, regional information is provided from the regional inflection points and its topographic visualization (Figs 3, 4 and 5). The histographic presentation (Fig. 3) shows that airway pressure needed to recruit all lung regions and pressure to induce hyperinflation might be close by or even overlap, so that lung collapse and hyperinflation may occur simultaneously in different regions. These findings might be helpful in the understanding of the underlying mechanisms of regional lung collapse and hyperinflation occurring during ventilatory strategies guided by global PV curves. Nevertheless, the regional LIPs show that airway pressure to keep all lung regions open is underestimated from the global measurements. Additionally, the UIP is underestimated by the global measurements.

\section{Methodogical aspects}

For the construction of regional PV curves, we used the technique of a low-flow manoeuvre in order to minimize the influence of airway resistance on lung mechanics. This method is simple, reproducible and allows good visualization of the initial part of the PV curve (12). Thus, during the low-flow manoeuvre, an on-line visualization of global and regional PV-curves was possible. In contrast, other techniques such as super syringe (37), flow interruption (38), PEEP-Wave (39), static compliance by automated single steps (27) and dynamic loop (40) 


\section{J. Hinz et al.}

should be possible as well, but require an off-line preparation of the measured data.

One methodological limitation is that EIT limits the assessment of regional PV curves to a single transverse thoracic plane with an estimated thickness of approximately $3 \mathrm{~cm}(41)$ and an estimated regional volume of approximately $8 \%$ of the thorax diameter (42) [9-29 ml, respectively (41)]. It has been demonstrated, that aeration gradients are found not only in the anterior-posterior but also in the cephalo-caudal direction $(6,28)$. During the low flow manoeuvre, the lungs may displace in the caudal direction, so that EIT is constantly measuring different lung planes. This is the same criticism against computed tomography studies, which have been shown to be misleading in the presence of cranial to caudal heterogeneity. Therefore, analysing regional PV curves in a single transversal plane may underestimate heterogeneity of PV curves of the whole lung.

We conclude that in patients with acute respiratory failure, global pressure volume curves do not reflect regional PV curves measured by EIT.

\section{Acknowledgements}

This study was supported by departmental funds and by Dräger AG (Dräger AG, Lübeck, Germany).

\section{References}

1. Gattinoni L, Pesenti A, Caspani ML, Pelizzola A, Mascheroni D, Marcolin $\mathrm{R}$ et al. The role of total static lung compliance in the management of severe ARDS unresponsive to conventional treatment. Intensive Care Med 1984; 10: 121-6.

2. Amato MB, Barbas CS, Medeiros DM, Magaldi RB, Schettino GP, Lorenzi-Filho $G$ et al. Effect of a protective-ventilation strategy on mortality in the acute respiratory distress syndrome. $N$ Engl J Med 1998; 338: 347-54.

3. Kunst PW, Bohm SH, de Vazquez A, Amato MB, Lachmann B, Postmus PE et al. Regional pressure Volume curves by electrical impedance tomography in a model of acute lung injury. Crit Care Med 2000; 28: 178-83.

4. van Genderingen HR, van Vught AJ, Jansen JR. Regional lung Volume during high-frequency oscillatory ventilation by electrical impedance tomography. Crit Care Med 2004; 32: 787-94.

5. van Genderingen HR, van Vught AJ, Jansen JR. Estimation of regional lung Volume changes by electrical impedance pressures tomography during a pressure-Volume maneuver. Intensive Care Med 2003; 29: 233-40.

6. Puybasset L, Gusman P, Muller JC, Cluzel P, Coriat P, Rouby JJ. Regional distribution of gas and tissue in acute respiratory distress syndrome. III. Consequences for the effects of positive end-expiratory pressure. CT Scan ARDS Study Group. Adult Respiratory Distress Syndrome. Intensive Care Med 2000; 26: 1215-27.

7. Rouby JJ, Lu Q, Goldstein I. Selecting the right level of positive end-expiratory pressure in patients with acute respiratory distress syndrome. Am J Respir Crit Care Med 2002; 165: 1182-6.
8. Albaiceta GM, Taboada F, Parra D, Luyando LH, Calvo J, Menendez $\mathrm{R}$ et al. Tomographic study of the inflection points of the pressure-Volume curve in acute lung injury. Am J Respir Crit Care Med 2004; 170: 1066-72.

9. Rouby JJ. Optimizing lung aeration in positive end-expiratory pressure. Am J Respir Crit Care Med 2004; 170: 1039-40.

10. Ramsay MA. Measuring level of sedation in the intensive care unit. JAMA 2000; 284: 441-2.

11. Artigas A, Bernard GR, Carlet J, Dreyfuss D, Gattinoni L, Hudson $\mathrm{L}$ et al. The American-European Consensus Conference on ARDS, part 2. Ventilatory, pharmacologic, supportive therapy, study design strategies and issues related to recovery and remodeling. Intensive Care Med 1998; 24: 378-98.

12. Mankikian B, Lemaire F, Benito S, Brun-Buisson C, Harf A, Maillot JP et al. A new device for measurement of pulmonary pressure-Volume curves in patients on mechanical ventilation. Crit Care Med 1983; 11: 897-901.

13. Barber DC, Brown BH. Applied potential tomography. J Phys E Sci Instrum 1984; 17: 723-33.

14. Hinz J, Neumann P, Dudykevych T, Anderson LG, Wrigge H, Burchardi $\mathrm{H}$ et al. Regional ventilation by electrical impedance tomography: a comparison with ventilation scintigraphy in pigs. Chest 2003; 124: 314322.

15. Hahn G, Frerichs I, Kleyer M, Hellige G. Local mechanics of the lung tissue determined by functional EIT. Physiol Meas 1996; 17 (Suppl. 4A): A159-A66.

16. Frerichs I, Hinz J, Herrmann P, Weisser G, Hahn G, Dudykevych $\mathrm{T}$ et al. Detection of local lung air content by electrical impedance tomography compared with electron beam CT. J Appl Physiol 2002; 93: 660-6.

17. O'Keefe GE, Gentilello LM, Erford S, Maier RV. Imprecision in lower 'inflection point' estimation from static pressurevolume curves in patients at risk for acute respiratory distress syndrome. J Trauma 1998; 44: 1064-8.

18. Venegas JG, Harris RS, Simon BA. A comprehensive equation for the pulmonary pressure-volume curve. J Appl Physiol 1998; 84: 389-395.

19. Murray JF, Matthay MA, Luce JM, Flick MR. An expanded definition of the adult respiratory distress syndrome. Am Rev Respir Dis 1988; 138: 720-3.

20. Crotti S, Mascheroni D, Caironi P, Pelosi P, Ronzoni G, Mondino $\mathrm{M}$ et al. Recruitment and derecruitment during acute respiratory failure: a clinical study. Am J Respir Crit Care Med 2001; 164: 131-40.

21. Hickling KG. The pressure-volume curve is greatly modified by recruitment. A mathematical model of ARDS lungs. Am J Respir Crit Care Med 1998; 158: 194-202.

22. Matamis D, Lemaire F, Harf A, Brun-Buisson C, Ansquer JC, Atlan G. Total respiratory pressure-volume curves in the adult respiratory distress syndrome. Chest 1984; 86: 58-66.

23. Slutsky AS, Tremblay LN. Multiple system organ failure. Is mechanical ventilation a contributing factor? Am J Respir Crit Care Med 1998; 157 (6 Part 1): 1721-25.

24. Plotz FB, Vreugdenhil HA, van Vught AJ, Heijnen CJ. Mechanical ventilation and multiple organ failure. Lancet 2003; 361 (9369): 1654.

25. Plotz FB, Slutsky AS, van Vught AJ, Heijnen CJ. Ventilatorinduced lung injury and multiple system organ failure: a critical review of facts and hypotheses. Intensive Care Med 2004; 30: 1865-72.

26. Sydow M, Burchardi H, Zinserling J, Ische H, Crozier TA, Weyland W. Improved determination of static compliance by automated single Volume steps in ventilated patients. Intensive Care Med 1991; 17: 108-14.

27. Sydow M, Burchardi H, Zinserling J, Crozier TA, Denecke T, Zielmann S. Intrinsic PEEP determined by static pressure- 
Volume curves - application of a novel automated occlusion method. Intensive Care Med 1993; 19: 166-71.

28. Wrigge H, Zinserling J, Neumann P, Defosse J, Magnusson A, Putensen $\mathrm{C}$ et al. Spontaneous breathing improves lung aeration in oleic acid-induced lung injury. Anesthesiology 2003; 99: 376-84.

29. Gattinoni L, Pelosi P, Crotti S, Valenza F. Effects of positive end-expiratory pressure on regional distribution of tidal Volume and recruitment in adult respiratory distress syndrome. Am J Respir Crit Care Med 1995; 151: 1807-14.

30. Gattinoni L, Pesenti A, Avalli L, Rossi F, Bombino M. Pressure-volume curve of total respiratory system in acute respiratory failure. Computed tomographic scan study. Am Rev Respir Dis 1987; 136: 730-6.

31. Pelosi P, D'Andrea L, Vitale G, Pesenti A, Gattinoni L. Vertical gradient of regional lung inflation in adult respiratory distress syndrome. Am J Respir Crit Care Med 1994; 149: 8-13.

32. Hahn G, Thiel F, Dudykevych T, Frerichs T, Gersing E, Schroder T et al. Quantitative evaluation of the performance of different electrical tomography devices. Biomed Tech (Berl) 2001; 4: 91-5.

33. Victorino JA, Borges JB, Okamoto VN, Matos GF, Tucci MR, Caramez MP et al. Imbalances in regional lung ventilation: a validation study on electrical impedance tomography. Am J Respir Crit Care Med 2004; 169: 791-800.

34. Wolf GK, Arnold JH. Noninvasive assessment of lung volume: respiratory inductance plethysmography and electrical impedance tomography. Crit Care Med 2005; 33 (Suppl. 3): S163-9.

35. Arnold JH. Electrical impedance tomography: on the path to the Holy Grail. Crit Care Med 2004; 32: 894-5.

36. Frerichs I, Dargaville PA, Dudykevych T, Rimensberger PC. Electrical impedance tomography: a method for monitoring regional lung aeration and tidal Volume distribution? Intensive Care Med 2003; 29: 2312-6.
37. Mead J, Whittenberger JL, Radford EP. Surface tension as a factor in pulmonary volume-pressure hysteresis. I Appl Physiol 1957; 2: 191-6.

38. Gottfried SB, Rossi A, Higgs BD, Calverley PM, Zocchi L, Bozic $C$ et al. Noninvasive determination of respiratory system mechanics during mechanical ventilation for acute respiratory failure. Am Rev Respir Dis 1985; 131: 414-20.

39. Putensen C, Baum M, Putz G. PEEP-Welle. Ein automatisiertes Verfahren zur bettseitigen Bestimmung der Volume $\mathrm{n} /$ Druck-Beziehung der Lunge beatmeter Patienten. Anaesthesist 1989; 38: 214-9.

40. Lu Q, Vieira SR, Richecoeur J, Puybasset L, Kalfon P, Coriat P et al. A simple automated method for measuring pressurevolume curves during mechanical ventilation. Am J Respir Crit Care Med 1999: 159: 275-82.

41. Hahn G, Hartung C, Hellige G. 6.2.5 Bestimmung der Grösse minimal erfassbarer Areale mit Ventilationsstörungen from: Elektrische Impedanztomographie (EIT) als Methode zur regionalen Beurteilung der Lungenventilation. Gustav Fischer Verlag, Mainz 1998; 77-77.

42. Brown $\mathrm{BH}$, Barber DC. Electrical impedance tomography; the construction and application to physiological measurement of electrical impedance images. Med Prog Technol 1987; 13: $69-75$.

Address:

J. Hinz

Robert-Koch-Str. 40

D-37075 Goettingen

Germany

e-mail: mail@josehinz.de 